\title{
Diffuse interstellar bands in Upper Scorpius: probing variations in the DIB spectrum due to changing environmental conditions ${ }^{\star}, \star \star$
}

\author{
D. A. I. Vos ${ }^{1}$, N. L. J. $\mathrm{Cox}^{2}$, L. Kaper ${ }^{3}$, M. Spaans ${ }^{4}$, and P. Ehrenfreund ${ }^{5}$
}

\author{
1 Radboud University Nijmegen, Toernooiveld 1, Postbus 9010, 6500 GL Nijmegen, The Netherlands \\ e-mail: nick.cox@ster.kuleuven.be \\ 2 Instituut voor Sterrenkunde, K.U. Leuven, Celestijnenlaan 200D, bus 2401, 3001 Leuven, Belgium \\ 3 Astronomical Institute "Anton Pannekoek", Universiteit van Amsterdam, Postbus 94249, 1090 GE Amsterdam, The Netherlands \\ 4 Kapteyn Astronomical Institute, Rijksuniversiteit Groningen, Postbus 800, 9700 AV Groningen, The Netherlands \\ 5 Astrobiology Group, Leiden Institute of Chemistry, Leiden University, Einsteinweg 55, 2300 RA Leiden, The Netherlands
}

Received 7 March 2008 / Accepted 28 July 2011

\begin{abstract}
Aims. We study the effects of local environmental conditions affecting the diffuse interstellar band (DIB) carriers within the Upper Scorpius subgroup of the Sco OB2 association. The aim is to reveal how the still unidentified DIB carriers respond to different physical conditions prevailing in interstellar clouds, in order to shed light on the origin of the DIB carriers.

Methods. We obtained optical spectra with FEROS on the ESO $1.52 \mathrm{~m}$ telescope at La Silla, Chile, and measured the equivalent widths of five DIBs (at 5780, 5797, 6196, 6379, and $6613 \AA$ ) as well as those of absorption lines of di-atomic molecules $\left(\mathrm{CH}, \mathrm{CH}^{+}, \mathrm{CN}\right)$ and atoms (K I, Ca I) towards 89 targets in the direction of Upper Scorpius. We construct a simple radiative transfer and chemical network model of the diffuse interstellar medium (ISM) sheet in front of Upp Sco to infer the effective radiation field.

Results. By measuring the DIB and molecular spectrum of diffuse clouds towards 89 sightlines in the Upper Scorpius region, we have obtained a valuable statistical dataset that provides information on the physical conditions that influence the band strengths of the DIBs. Both the interstellar radiation field strength, $I_{\mathrm{UV}}$, and the molecular hydrogen fraction, $f_{\mathrm{H}_{2}}$, have been derived for 55 sightlines probing the Upp Sco ISM. We discuss the relations between DIB strengths, $\mathrm{CH}$ and $\mathrm{CH}^{+}$line strengths, $E_{(B-V)}, I_{\mathrm{UV}}$, and $f_{\mathrm{H}_{2}}$. The ratio between the 5780 and $5797 \AA$ DIBs reveals a (spatial) dependence on the local environment in terms of cloud density and exposure to the interstellar radiation field, reflecting the molecular nature of these DIB carriers.
\end{abstract}

Key words. astrochemistry - ISM: clouds - ISM: lines and bands - dust, extinction - ISM: individual objects: Upper Scorpius ISM: molecules

\section{Introduction}

The diffuse interstellar medium contains compounds of unidentified origin that absorb in the UV-visual to near-infrared spectral range. More than 300 different diffuse interstellar bands (DIBs) are currently identified (Herbig 1995; Hobbs et al. 2008). Many possible carriers have been proposed, ranging from grain impurities and exotic molecules to $\mathrm{H}_{2}$. In the past two decades the field has converged towards larger carbonaceous molecules, like the fullerenes and polycyclic aromatic hydrocarbons (PAHs), which have electronic transitions in the optical (see for example Salama et al. 1999; Ruiterkamp et al. 2005; Kokkin \& Schmidt 2006; Zhou et al. 2006; and Salama et al. 2011). New diffuse bands have been detected in one line-of-sight which appear to match with naphthalene and anthracene cations (Iglesias-Groth et al. 2008, 2010) and the weak $5450 \AA$ DIB is found to match with an absorption band arising from a hydrocarbon plasma created in the laboratory (Linnartz et al. 2010). Linear- $\mathrm{C}_{3} \mathrm{H}_{2}$ has been put forward as a carrier of the 5450 and $4881 \AA$ DIBs

^ Based on observations collected at the European Southern Observatory, Paranal, Chile (ESO program 63.H-0456).

$\star \star$ Tables 1, 2, and 5, and Appendices are available in electronic form at http://www . aanda.org by Maier et al. (2011). These assignments are, however, tentative and disputed (Galazutdinov et al. 2011).

In order to understand the chemical and physical properties of the DIB carrier(s) it is important to study their behaviour in different interstellar environments, both in our own galaxy and beyond. Studies of DIBs in the Magellanic Clouds (Ehrenfreund et al. 2002; Cox et al. 2006, 2007; Welty et al. 2006), M 31 (Cordiner et al. 2008a,b) and beyond (e.g. Heckman \& Lehnert 2000; York et al. 2006; Sollerman et al. 2005; Cox \& Patat 2008) illustrate that DIB carrier abundances (per amount of dust and gas) can be similar to Galactic values. However, these studies have also revealed systematic differences in these extragalactic environments.

A large amount of published information is available regarding DIBs in many sightlines probing the Galaxy (e.g. Herbig 1993; Chlewicki et al. 1986; Krełowski et al. 1999; Thorburn et al. 2003; Galazutdinov et al. 2004; Weselak et al. 2004, 2008b; Friedman et al. 2011), yielding relations of DIB properties with respect to each other and to other diffuse ISM gas and dust tracers. Most of these studies focused on DIBs probing various galactic environments, and provided average results for the Milky Way. Studies dealing with a particular region usually only include a very limited number of sightlines. One exception is the study of the Orion region by Jenniskens et al. (1994) which 
entails 22 lines of sight. Another multi-object study, by van Loon et al. (2009), used the globular cluster $\omega$ Cen to probe fluctuations of CaII, NaI and the $\lambda \lambda 5780$ and 5797 DIBs in the diffuse - low reddening - foreground ISM. This study revealed small-scale structure - on parsec scales - in the warm neutral and weakly ionised medium of the Disc-Halo interface. The observed low 5797/5780 DIB ratio was found to be consistent with the relative high UV radiation levels typically inferred for the extra-planar warm medium.

Nearby OB associations host many bright early-type stars confined in a relatively small area of the sky. These stars have only few stellar lines in the optical spectrum contaminating the interstellar spectrum. Thus, these associations provide a setup that is perfectly suited to study the effect of varying local conditions on the DIB spectrum. One of these associations, Scorpius OB2, is a young (5-20 Myr), low-density $\left(\approx 0.1 M_{\odot} \mathrm{pc}^{-3}\right)$ grouping of stars divided in three subgroups (de Zeeuw et al. 1999; Kouwenhoven et al. 2005). Scorpius (Upp Sco) region is the subgroup near the Ophiuchus star forming region and the $\rho$ Oph cloud at a distance of $145 \pm 2 \mathrm{pc}$ (de Zeeuw et al. 1999). Combining 2MASS extinction maps with Hipparcos and Tycho parallaxes, Lombardi et al. (2008) found a distance of $119 \pm 6 \mathrm{pc}$ for the $\rho$ Ophiuchi cloud (with the core at $128 \pm 8 \mathrm{pc}$ ). Mamajek (2008) suggested a mean distance of $139 \pm 6 \mathrm{pc}$ for the distance of the Ophiuchus molecular cloud, which they placed within $11 \mathrm{pc}$ of the centroid of the Upper Scorpius subgroup.

Filamentary - interstellar - material connected to the $\rho$ Ophiuchus cloud complex is observed towards Upper Scorpius (de Geus 1992). The densest part of this complex is the $\rho$ Oph dark cloud, a site of ongoing low-mass star formation (Grasdalen et al. 1973; Greene \& Young 1992; Wilking et al. 1997; Preibisch $\&$ Zinnecker 2001) that is exposed to the radiation fields and stellar winds produced by nearby early-type stars. A detailed review on the stellar population and star formation history of the Sco OB2 association is given by Preibisch \& Mamajek (2008) and Wilking et al. (2008).

The advantages of studying the properties of DIBs in the Upper Scorpius region are numerous. It is in close vicinity and it has been extensively studied in the past. Detailed information is available on both the stellar content (spectral types, photometry, distances, kinematics, etc.) and the conditions of the surrounding interstellar medium (dust emission and absorption, IR-to-farUV extinction curves, UV emission, molecular content, etc.). It exhibits a significant variation in local environmental conditions which should translate into changing properties of the DIBs (if they depend on these conditions) when probing different parts of the Upp Sco region.

Previous studies of interstellar gas and dust in the Upp Sco region focused on the $\rho$ Oph cloud and a few other nearby bright B stars. Snow et al. (2008) give a concise summary of different studies of the Upp Sco region covering a range of topics including UV extinction, atomic and molecular hydrogen, atomic and molecular gas, astrochemistry, and DIBs. For example, $\mathrm{H}_{2}$ observations show that sightlines in this region have both low $(\leq 0.1)$ and high $(\sim 0.3-0.6)$ molecular fractions $f_{\mathrm{H}_{2}}$ (e.g. Savage et al. 1977).

In this paper we investigate the behaviour of five well-known DIBs (at 5780, 5797, 6196, 6379, and $6613 \AA$ ) and the molecular lines of $\mathrm{CH}, \mathrm{CH}^{+}$, and $\mathrm{CN}$ in the sightlines towards 89 B-type stars in the direction of Upp Sco (Fig. 1). These targets, within a field of $20^{\circ} \times 20^{\circ}$, provide a unique and detailed view of the gas and dust in this nearby association. In Sect. 2 we introduce our sample and provide information on the reduction of the obtained spectra. Section 3 briefly discusses line-of-sight reddening and dust towards Upp Sco. In Sect. 4 we present the observational details of atomic and molecular lines as well as diffuse bands. We explore the results in Sect. 5, where we discuss first the relation between DIB strength, the dust tracer $E_{(B-V)}$, and the molecular content. Then, we demonstrate that the DIB ratio 5797/5780 may be useful to distinguish between lines-of-sight probing diffuse cloud edges and those penetrating denser cloud cores. The observed differences in physical properties of both types of sightlines are often attributed to the skin effect, the increase in effective shielding of molecules from UV radiation as one moves deeper into an interstellar cloud (Krełowski \& Westerlund 1988; Herbig 1995; Cami et al. 1997). Furthermore, we have studied the effect of local environmental conditions, such as density and UV field strength on DIB strengths and ratios. The line strengths of $\mathrm{CH}, \mathrm{CH}^{+}$, and $\mathrm{CN}$ can be used to characterise the physical and chemical conditions in the respective sightlines. We have constructed a simple dust cloud model to derive the intensity of the interstellar radiation field (ISRF) from the observed $\mathrm{CH}$ and $\mathrm{CN}$ line strengths. The paper concludes with a summary of the main results (Sect. 6).

\section{Optical spectra of B-type stars in Upp Sco}

The observed targets cover a region in the sky of approximately $20^{\circ} \times 20^{\circ}$ (i.e. $\sim 50 \times 50 \mathrm{pc}$ at a distance of $145 \mathrm{pc}$ ). Within this relatively small region 89 sightlines are measured and analysed. The positions of the observed targets in the Upper Scorpius region are shown on a $100 \mu \mathrm{m}$ image (far-infrared dust reddening map; Fig. 1), a reprocessed composite of the COBE/DIRBE and IRAS/ISSA maps with the zodiacal foreground and confirmed point sources removed (Schlegel et al. 1998).

Seven out of eight stars that generate $90 \%$ of the local interstellar radiation field (ISRF) are located in this region. The dust in these lines-of-sight imposes $E_{(B-V)}$ values from $\sim 0.02$ up to $\sim 0.99$ mag (see Sect. 3), implying local variations in the cloud column or volume - density and structure, and subsequently the attenuation of the ISRF. Therefore this large dataset is extremely valuable to investigate the effects of environmental conditions on the DIB carriers on a local scale.

Echelle spectra were obtained with the FEROS instrument on the ESO $1.52 \mathrm{~m}$ telescope at La Silla from 26-30 April 1999. The spectra were taken at a resolving power of $R \approx 48000 \mathrm{cov}$ ering a spectral range of 3800 to $8500 \AA$. The data were reduced using the FEROS context within the ESO-MIDAS data reduction package. Data reduction was performed in a standard fashion, the CCD images were first bias subtracted and subsequently the Echelle orders were straightened, extracted, unblazed (flat fielded), rebinned (wavelength calibrated) and finally merged. We extracted and normalised the spectral ranges of interest. Final spectra have signal-to-noise ratios between 100 and 400 in the wavelength regions of the measured lines. Typically, the $\mathrm{S} / \mathrm{N}$ values are lower for the blue region ( 3900-4500 $\AA$ ) with respect to the red part of the spectrum. Furthermore, exact values differ for each line-of-sight (due to differences in exposure time, visual magnitude of the star and the weather conditions). The $\mathrm{S} / \mathrm{N}$ is reflected in the reported equivalent width uncertainties.

Table 1 summarises the basic data for the observed targets: Henry-Draper (HD) and Hipparcos (HIP) number, spectral type, right ascension and declination, colour $B-V$, intrinsic colour $(B-V)_{0}$, reddening $E_{(B-V)}$ (see Sect. 4), total-to-selective visual extinction $R_{\mathrm{V}}$, and Hipparcos distance (pc). 


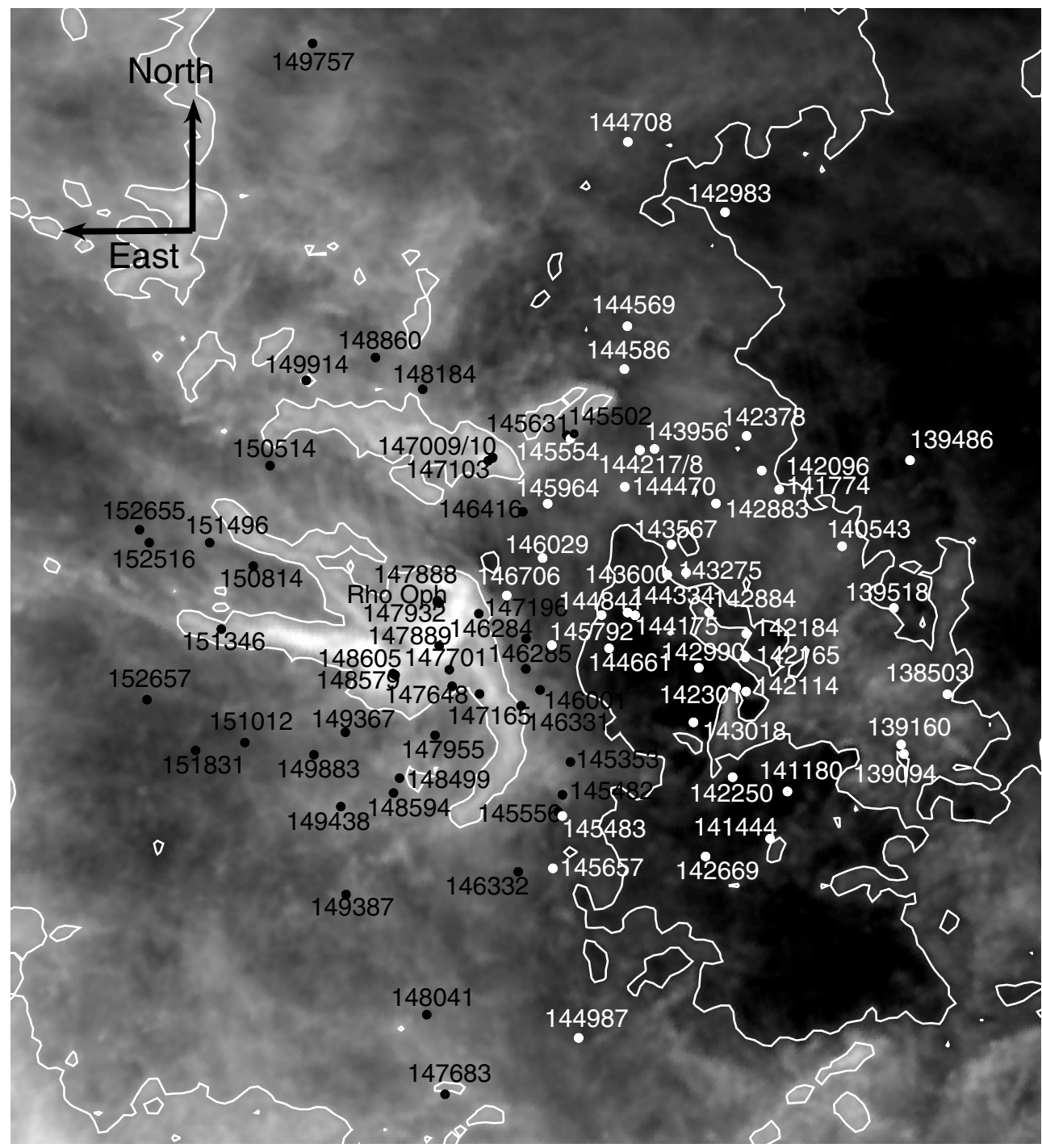

Fig. 1. The positions (black and white dots) of the 89 mainly B-type members of Upp Sco are shown (with HD numbers) on top of a $100 \mu \mathrm{m}$ infrared dust map of this region (Schlegel et al. 1998). The north-east arrows are $3^{\circ}$ in length. The $\rho$ Oph cloud can be identified by the bright filamentary emission located just left to the center of the figure. The dust emission (on a logarithmic grey scale) is proportional to the reddening $E_{(B-V)}$ of sightlines penetrating these clouds; the lowest intensities correspond to $E_{(B-V)} \sim 0.02$ mag (black) and highest intensities to $\geq 2 \mathrm{mag}$ (white). The well-known targets $\sigma$ Sco (HD 147165, in the $\rho$ Oph cloud) and $\zeta$ Oph (HD 149757, top left) are included in our study.

\section{Line-of-sight reddening and dust}

For each target we derive the reddening $E_{(B-V)}$ from the $B$ and $V$ photometry (taken from Tycho-2 and converted to Johnson system) after assigning the intrinsic colour $(B-V)_{0}$ (from Fitzgerald 1970) according to the spectral type of the target (as provided by the Michigan Spectral Catalog of HD stars; Houk 1982; Houk \& Smith-Moore 1988). Visual inspection of the spectral range from 4000 to $5000 \AA$, used for the classification of OB-type stars (Walborn \& Fitzpatrick 1990), gives results in good agreement with the spectral types listed in the Michigan Spectral Catalog. The adopted magnitudes and results for $E_{(B-V)}$ are listed in Table 1 . The total error for $E_{(B-V)}$ is $\sim 0.03 \mathrm{mag}$, which is derived from the error of the Tycho- $2 B$ and $V$ photometry ( $0.02 \mathrm{mag})$, the assumed uncertainty $(\sim 0.01 \mathrm{mag})$ in the transformation to the Johnson system, the colour range of spectral sub-types, and the uncertainty in the spectral classification (both $\sim 0.01$ mag for our B stars).

Two structures of interstellar medium are observed towards the Upp Sco complex. A recent study of the distribution and motions of the interstellar gas in the $\rho$ Oph region provides evidence for a low density/extinction ISM component, at a distance of 50-80 pc, located in front of the $\rho$ Oph complex (Snow et al. 2008). This nearest sheet-like structure was also observed at a distance of $\sim 60 \mathrm{pc}$ towards the Sco-Cen region by Corradi et al. (2004). This structure has a very low column density and an almost negligible effect on the observed reddening.

The second structure is located at a distance of $\sim 110-150 \mathrm{pc}$, consisting of diffuse extended portions of the dense $\rho$ Oph cloud at $122 \pm 8 \mathrm{pc}$ (Snow et al. 2008). This is consistent with a mean thickness of $\sim 30$ pc found by Lombardi et al. (2008). Combined with the gas densities measured by Zsargó \& Federman (2003), which suggest a cloud thicknesses between 1 and $15 \mathrm{pc}$, this implies that these clouds are not spread homogeneously throughout the Upp Sco region but form a patchy complex of scattered and loosely connected clouds.

Note that the column density of this dust sheet $\left(N(\mathrm{H}) \sim 3.2-50 \times 10^{20} \mathrm{~cm}^{-2}\right.$; Bohlin et al. 1978; Diplas \& Savage 1994) located at approximately $125 \mathrm{pc}$ is an order of magnitude higher than that of the nearer sheet. For additional 


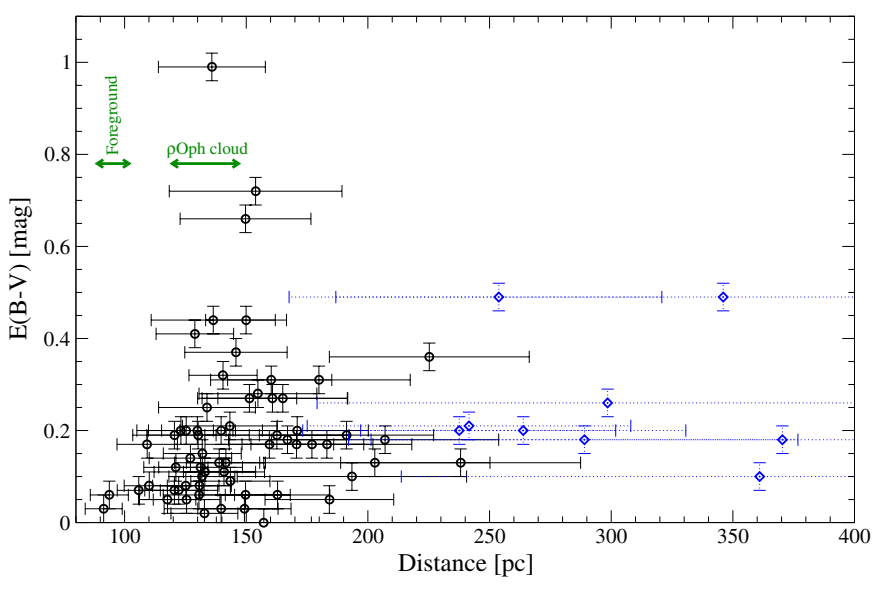

Fig. 2. The colour excess $E_{(B-V)}(\mathrm{mag})$ is plotted against the distance of the observed targets (Perryman et al. 1997). Stars with distance errors larger than $50 \mathrm{pc}$ are shown as squares in grey/blue. The low $E_{(B-V)}$ below $120 \mathrm{pc}$ indicates that there is little foreground material in front of Upper Scorpius. The increased scatter at $140 \pm 20 \mathrm{pc}$ reflects the density variation associated primarily with the $\rho$ Oph cloud complex. Beyond that distance, no substantial increase of reddening is observed up to $400 \mathrm{pc}$. A similar figure for the $\rho$ Oph cloud region was shown in Lombardi et al. (2008) (also based on optical photometry, with a partial overlap in the selected sky region).

information on interstellar material observed towards the $\rho$ Oph molecular cloud complex see also Motte et al. (1998).

The above is supported by the measured colour excess $E_{(B-V)}$ as a function of target distance (Fig. 2). Six stars are probably in front of Upp Sco while about 10 to 15 of these are background stars. The strong increase of the reddening around a distance of $140 \mathrm{pc}$ suggests that most material contributing to the extinction is associated with the Upp Sco complex, with the observed scatter resulting from variations in the - column or volume - density within this region. The reddening values extracted directly from the dust reddening map (Schlegel et al. $1998)$ are compared with the $E_{(B-V)}$ values obtained from optical photometry and stellar classification for the individual sightlines (Fig. 3). We note, however, that extinction maps based on infrared emission or optical/near-infrared star counts show systematic offsets with respect to each other and are unreliable at small scales $\left(\leq 5^{\prime}\right)$, with typical $1 \sigma$ uncertainties of $1.2 \mathrm{mag}$ in $A_{\mathrm{V}}$ (e.g. Schnee et al. 2005). For all sightlines the reddening inferred from far-infrared emission is higher than that derived from optical photometry and spectroscopy, which suggests that, for most sightlines, the infrared emission also traces dust that is located behind the observed star. Some caution is required comparing these two results as some variation would be expected due to calibration/systematic and statistical errors on both values. Nevertheless, most sightlines with $E_{(B-V) \text { optical }}<0.3 \mathrm{mag}$ have $E_{(B-V) \text { infrared }}<0.6 \mathrm{mag}$, which is fully consistent with a dust sheet associated to the Upp Sco OB association, with both stars and dust inter-dispersed with each other. For sightlines in the direction of the dense $\rho$ Oph cloud much higher values for $E_{(B-V)}(>1 \mathrm{mag})$ are inferred from the dust map with respect to the optical photometric data. Logically, stars visible in this direction are likely situated at the front side of this dense cloud (the stars at the back will be much fainter/invisible due to higher extinction).

The contribution from foreground material to the observed total reddening is very small $\left(E_{(B-V)} \lesssim 0.02 \mathrm{mag}\right)$. Therefore, we conclude that the dust distribution inferred from both the

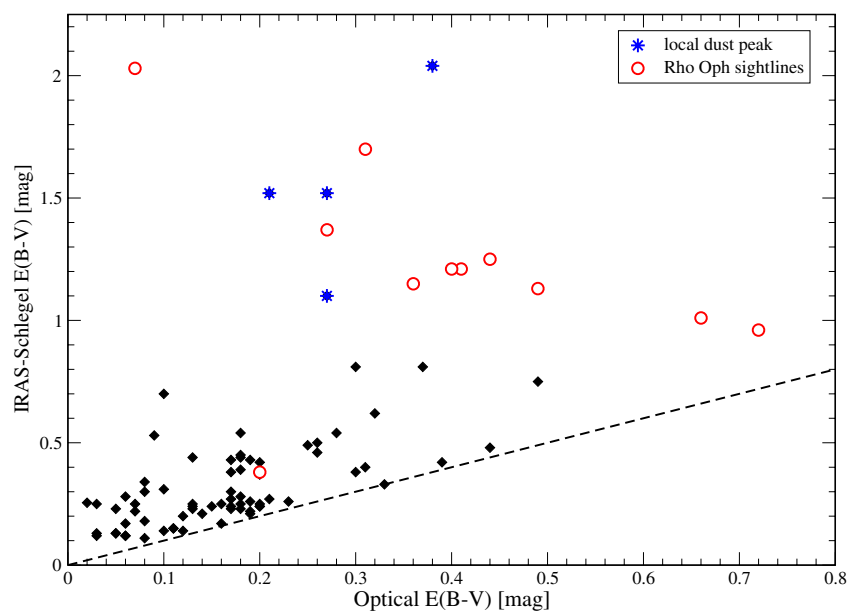

Fig. 3. Comparison of the amount of dust, as indicated by $E_{(B-V)}$, inferred from optical photometry observations and stellar spectral classification with those inferred from infrared dust emission (IRAS $100 \mu \mathrm{m}$ map; Schlegel et al. 1998). $\rho$ Oph sightlines are indicated separately as well as 4 sightlines connected to an infrared dust emission structure north of $\rho$ Oph. The dashed line indicates the 1-to-1 relation between the two measurements.

$100 \mu \mathrm{m}$ infrared emission and the line-of-sight reddening is predominantly due to the Upper Scorpius complex. In other words, the low-density foreground dust sheet contributes very little to the total observed values for the infrared emission and the reddening.

\section{Interstellar absorption lines}

In this section we present the properties of the interstellar absorption lines observed towards the 89 Upper Scorpius targets. We determined equivalent widths for the five strong DIBs at 5780, 5797, 6196, 6379, and $6613 \AA$, for the di-atomic lines of $\mathrm{CH}, \mathrm{CH}^{+}$, and $\mathrm{CN}$ as well as for the $\mathrm{K}_{\mathrm{I}}$ and $\mathrm{Ca} \mathrm{I}$ lines (Table 2). The Na I D doublet is omitted because it is saturated for the majority of sightlines. Line profiles and central heliocentric velocities for the atomic and di-atomic lines are given in Fig. D.1 and Table 5, respectively. To illustrate, the velocity absorption profiles of the atomic and molecular absorption lines towards HD 147889 are shown in Fig. 4.

\subsection{Diffuse interstellar bands}

Although more than 300 DIBs are known we focus here on the five strong and narrow bands at 5780, 5797, 6196, 6379, and $6613 \AA$. The strength and width of these features facilitates the measurement of modest column densities of their carriers in slightly reddened sightlines. Additionally, the Galactic relationships between DIB strength and reddening are well established for these DIBs both in the Galaxy and beyond. The equivalent width, $W$, is measured via a straight line continuum integration across the absorption feature (see Appendix. A). For the DIB measurement we do not expect significant contamination from stellar atmosphere lines (see Appendix. B). The measured equivalent widths, or $2 \sigma$ upper limits, are listed in Table 2 for the five DIBs towards the 89 targets in Upp Sco. This is the first consistently measured data set containing this many sightlines within one region.

To ascertain the accuracy and consistency of our results we compare our measured values with those available in the 
D. A. I. Vos et al.: Diffuse interstellar bands in Upper Scorpius

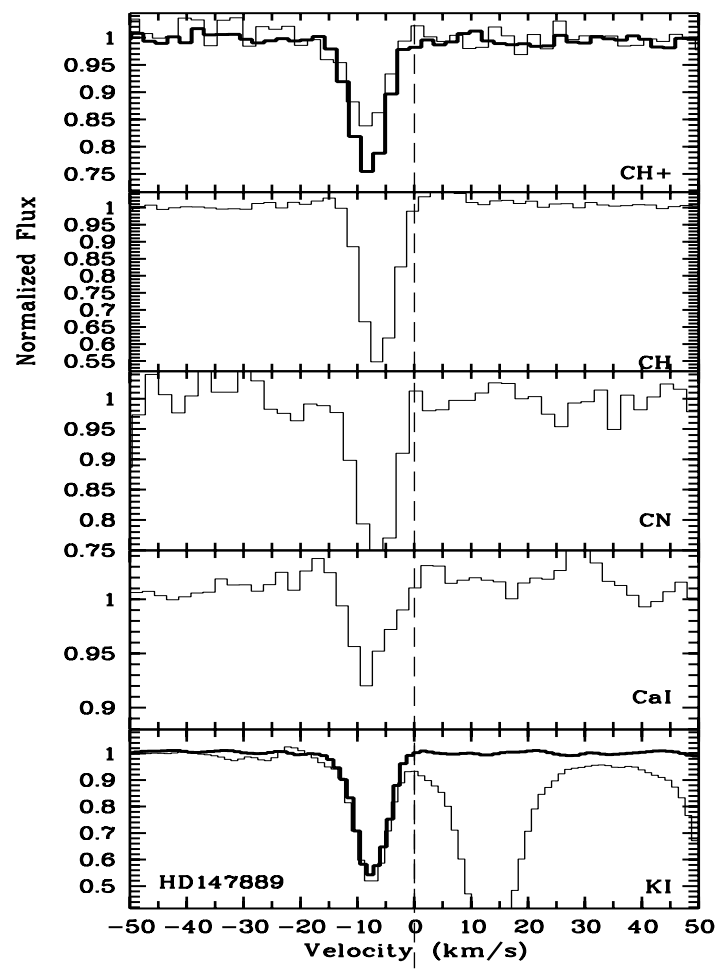

Fig. 4. Example of the velocity profiles of interstellar absorption lines. The line-of-sight shown is that towards HD 147889. From top to bottom the $\mathrm{CH}^{+}$(both at 3957.7 and $4232.5 \AA$, latter as thick line), $\mathrm{CH}$, CN R(0), CaI, and $\mathrm{K}_{\mathrm{I}}$ line (both 7665 and 7699, latter as thick solid line). The strong feature at $\sim 15 \mathrm{~km} \mathrm{~s}^{-1}$ in the bottom panel is a telluric absorption line. Similarly, velocity profiles for all sightlines are included in Appendix D.

literature (Herbig 1993; Seab \& Snow 1995; Megier et al. 2001; Galazutdinov et al. 2002; Thorburn et al. 2003; Galazutdinov et al. 2004; Megier et al. 2005; Sollerman et al. 2005; and Friedman et al. 2011). The result is shown in Fig. 5 for the 5780 and 5797 DIBs, and in Fig. 6 for the 6196, 6379, and $6613 \AA$ DIBs. The correlation between values from this work and from literature are good (for the 5 DIBs, the correlation coefficients $r$ range from 0.92 to 0.99 ). The linear regressions reveal a small non-zero offset, indicating that our values are systematically lower by a few percent. We note that for individual cases the values between different studies vary significantly by as much as $20 \%$. Small inconsistencies (between all studies) arise naturally from differences in the data quality $(\mathrm{S} / \mathrm{N}$, resolution) as well as differences in the adopted methods for equivalent width measurements (adopted stellar continuum, contamination from nearby weak features, adopted integration limits, inclusion or removal of underlying broad bands). In conclusion, the measured equivalent widths are consistent with previous studies but do show a small systematic offset.

Cami et al. (1997) found that the $\lambda \lambda 5797,6379$, and 6613 DIBs show a good correspondence to each other, with $r \sim 0.8$. These authors also found that the $\lambda 5780$ DIB is moderately correlated with the $\lambda 6613 \mathrm{DIB}(r=0.65)$ and weakly to the 6379 DIB ( $r=0.47)$. Recently, Friedman et al. (2011) found high values for $r$ (ranging from 0.93 to 0.99 ) for the Galactic DIB pairs in Table 3. McCall et al. (2010) reported a nearly perfect correlation $(r=0.99)$ between the $\lambda \lambda 6196$ and 6613 DIBs toward 114 Galactic diffuse cloud sightlines. In this work we find $r=0.8$ for $\lambda \lambda 6196-6613$ DIB pair, which is
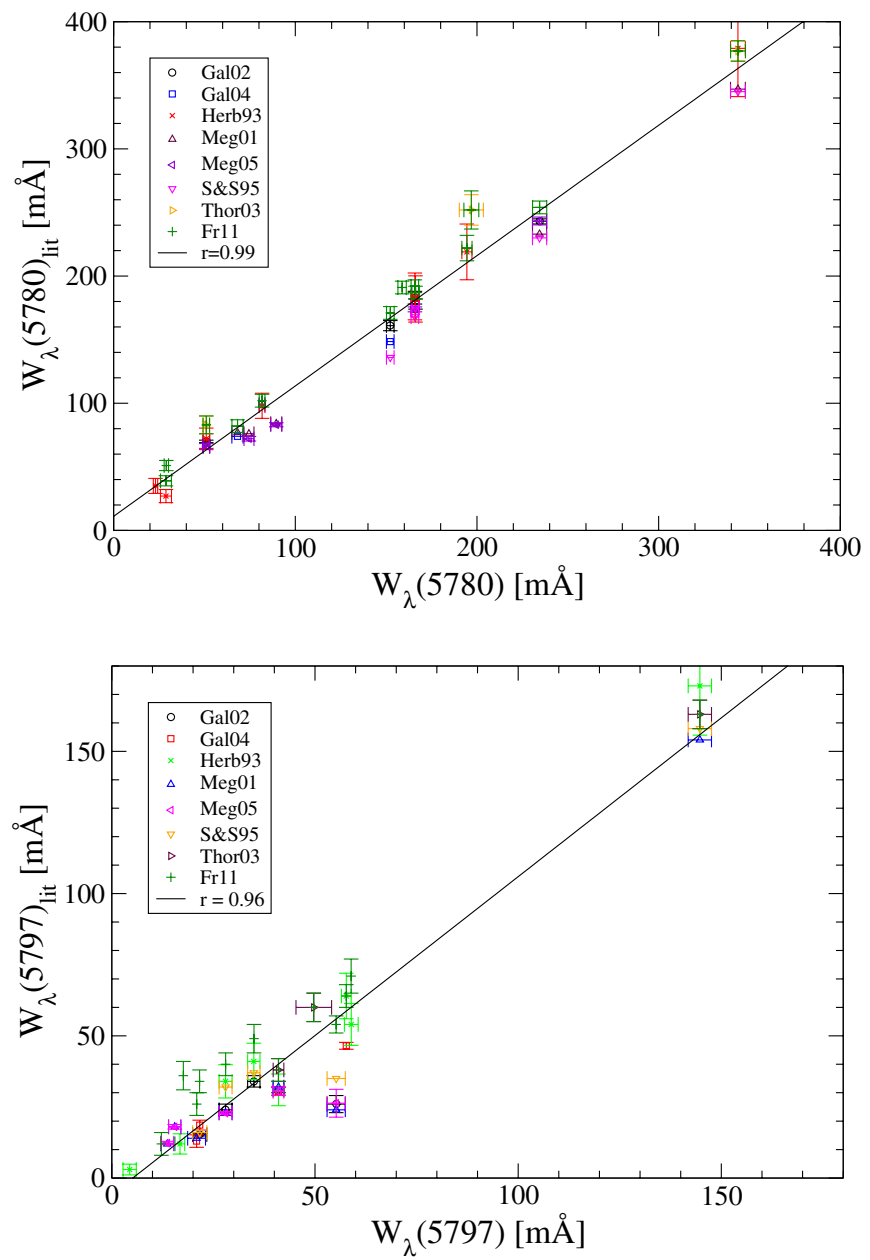

Fig. 5. Equivalent widths of the $\lambda \lambda 5780$ and 5797 DIBs previously measured for sightlines included in this survey compared to values measured in this work. For several sightlines several literature values are available, illustrating the "intrinsic" scatter in equivalent widths due to measurement methods. Literature values are taken from Herbig (1993); Seab \& Snow (1995); Galazutdinov et al. (2002); Thorburn et al. (2003); Galazutdinov et al. (2004); Sollerman et al. (2005); Megier et al. (2001, 2005); and Friedman et al. (2011). Linear regressions are shown in each panel. Correlation coefficients $r$ are 0.99 and 0.96 for the 5780 and $5797 \AA$ DIBs, respectively. Slopes and intercepts of these regressions are 1.02 and $11 \mathrm{~m} \AA$ for the $5780 \AA \mathrm{DIB}$ and 1.12 and $-6 \mathrm{~m} \AA$ for the $5797 \AA$ DIB, respectively.

less than for other pairs. The correlation coefficient between the five DIBs measured in this study are given in Table 3 . In line with previous results, the $\lambda 5797$ DIB has a good correlation with both $\lambda \lambda 6379$ and 6613 DIBs, however, it shows a poor correlation with both $\lambda \lambda 5780$ and 6196 DIBs. The $\lambda \lambda 6379$ and 6613 DIB pair shows the strongest correlation, with $r=0.92$. In fact, the $\lambda 6613$ DIB correlates well with all four DIBs. The $\lambda 5780$ DIB shows a good correlation with the $\lambda 6613$ DIB $(r=0.85)$. Unexpectedly, the other two DIB family members of $\lambda 6613$ (i.e. $\lambda \lambda 5797$ and 6379 DIBs) have a weaker correlation. Restricting the computation of $r$ to the 13 sightlines present in both Friedman et al. (2011) and this work, increases $r$ for our data (but lowers $r$ slightly for the Friedman sample). For example, for the $\lambda \lambda 5780-5797$ DIB pair $r=0.75$ (this work) and $r=0.86$ (Friedman); for the $\lambda \lambda 6196-6613$ DIB pair $r=0.97$ (this work) and $r=0.99$ (Friedman), and for the $\lambda \lambda 5780-6196$ DIB pair both studies give $r=0.93$ (for the 

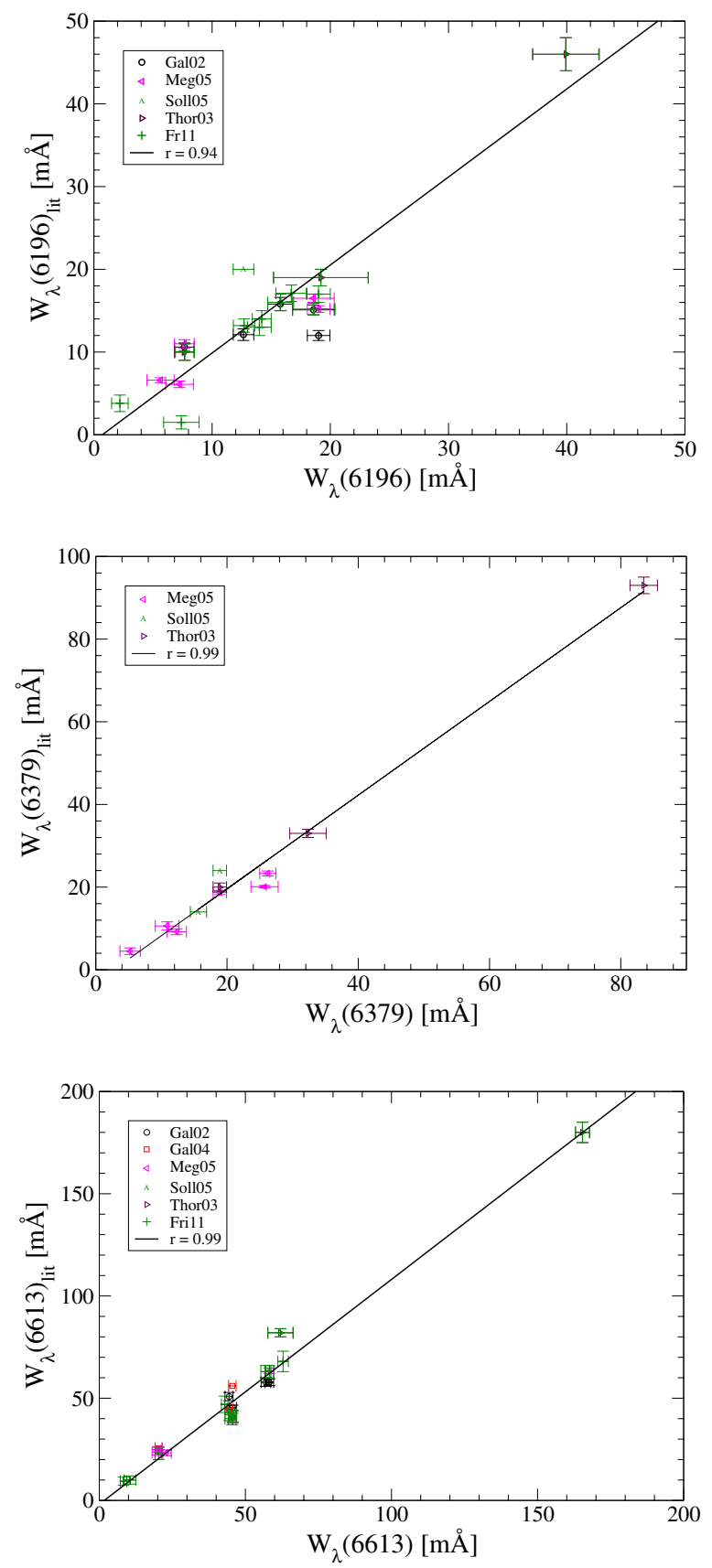

Fig. 6. The equivalent widths of the 6196,6379 , and $6613 \AA$ DIBs in lines-of-sight previously measured and included here are compared with the measurements obtained in this work. Literature values are taken from Herbig (1993); Seab \& Snow (1995); Galazutdinov et al. (2002); Thorburn et al. (2003); Galazutdinov et al. (2004); Sollerman et al. (2005); Megier et al. (2001, 2005). Linear regressions with the corresponding correlation coefficients $r$ are shown in each panel. Slopes and intercepts of these regressions are 1.06 and $-0.8 \mathrm{~m} \AA$, 1.13 and $-3.1 \mathrm{~m} \AA$, and 0.995 and $-1.9 \mathrm{~m} \AA$, for the 6196, 6379, and $6613 \AA$ DIBs, respectively.

complete dataset Friedman report $r=0.97)$. The higher Pearson correlation coefficient (independent of quoted error bars) for the Friedman data suggests that the overall uncertainties on the measurements are lower than for this work, resulting in an improved correlation. Partly this is due to the fact that our sample includes a large fraction of sightlines with low values for $E_{(B-V)}$, and thus weak DIBs. On the other hand, restricting the comparison to the
Table 3. Pearson correlation coefficients $r$ between the observed DIBs.

\begin{tabular}{lccccc}
\hline \hline DIB & 5780 & 5797 & 6196 & 6379 & 6613 \\
\hline 5780 & 1 & 0.72 & 0.74 & 0.75 & 0.85 \\
5797 & & 1 & 0.69 & 0.87 & 0.85 \\
6196 & & & 1 & 0.81 & 0.80 \\
6379 & & & & 1 & 0.92 \\
6613 & & & & & 1 \\
\hline
\end{tabular}

Upp Sco sightlines in common lowers $r$ in both samples (probably as there are fewer data points), and also reduces the difference between the two sets. This could be partly due to an increased effect of local variations in the DIB spectrum on the correlation coefficient (such effects would be averaged out in a larger Galactic survey probing many different regions as opposed to probing a peculiar region like Upp Sco).

\subsection{Molecular lines}

We have measured equivalent widths and heliocentric radial velocities for the $\mathrm{CH}\left(\lambda_{\text {rest }}=4300.313 \AA\right), \mathrm{CH}^{+}\left(\lambda_{\text {rest }}=\right.$ $4232.548 \AA)$, and $\mathrm{CN} \mathrm{R}(0)\left(\lambda_{\text {rest }}=3874.608 \AA\right)$ lines (Tables 2 and 5, respectively). In a few (about 5) cases, the CN (3874.608 $\AA$ ), $\mathrm{CH}(3886.410 \AA)$, and $\mathrm{CH}^{+}(3957.70 \AA)$ lines are tentatively detected (see e.g. Fig. 4). These lines are weak and have large $(>50 \%)$ uncertainties. It may be that the strongest $\mathrm{CH}$ line is saturated, which can occur for individual components with $W(\mathrm{CH}) \geq 20 \mathrm{~m} \AA$ (Van Dishoeck \& Black 1989). The $\mathrm{CN} \mathrm{R}(0)$ transition is also prone to saturation for (individual) components with $W>6 \mathrm{~m} \AA$, leading to underestimated column densities, though the corrections are less than about $20 \%$ up to $W=15 \mathrm{~m} \AA$ (Słyk et al. 2008). The CN lines toward HD 147683, HD 147701, HD 147889, and HD 147932 likely suffer from saturation. For the lines-of-sight including the strongest $\mathrm{CN}$ lines in our sample, HD 147932, HD 147701, and HD 147889, the column densities would need to be corrected by a factor 1.27, 1.7, and 2.1, respectively (following Słyk et al. 2008; adopting a value of $1 \mathrm{~km} \mathrm{~s}^{-1}$ for the Doppler broadening). Saturation also occurs for $\mathrm{CH}^{+}$if $W \geq 20$ or $\geq 40 \mathrm{~m} \AA$ for components with $b=1$ or $2 \mathrm{~km} \mathrm{~s}^{-1}$, respectively (Allen 1994). Only a few sightlines have measured total $W$ larger than these limits, and even for these cases the individual (unresolved) velocity components are not expected to be strongly saturated as noted above. For the sightlines towards HD 147683, HD 147889, HD 147933, and HD 149757 the equivalent width ratio between the (tentatively) detected weaker and stronger lines of both $\mathrm{CH}$ and $\mathrm{CH}^{+}$are close to - within the uncertainties - the expected ratio of $\sim 3.9$, and $\sim 1.9$, respectively. Only for the latter two sightlines are these ratios significantly lower $(\sim 1.5)$ indicative of some saturation.

For certain spectral types stellar line contamination can complicate measurements. However, for the majority of spectra presented here this problem could be well resolved (see also Appendix B). Equivalent widths for $\mathrm{CH}$ and $\mathrm{CH}^{+}$given by Federman et al. (1994); Megier et al. (2005), and Weselak et al. (2008b) for nine Upp Sco sightlines in common with this work are consistent with our values. Furthermore, the reported $W(\mathrm{CN})$ are consistent with values given by Słyk et al. (2008) for six targets in common, although their reported error bars are smaller. The velocity profiles for $\mathrm{CH}, \mathrm{CH}^{+}$, and $\mathrm{CN}$ are also shown, for the relevant sightlines, in Fig. D.1. 


\subsection{Atomic lines}

Inspection of the Na I (5889.951 \& $5895.924 \AA$; Morton 2003) and K I (7664.91 \& $7698.974 \AA$; Morton 2003) doublets shows that most (75 of 89) sightlines are dominated by one strong velocity component. This strong component displays asymmetries and broadening for a number of sightlines suggesting that in reality multiple unresolved narrow components may be present (see e.g. Sect. 5.5 and Snow et al. 2008). The obtained spectral resolution is not sufficient to resolve hyperfine splitting (order of $\sim 1 \mathrm{~km} \mathrm{~s}^{-1}$ ). For 10 sightlines (all with low reddening; $\left.E_{(B-V)} \lesssim 0.2\right)$ two or three weaker components, clearly separated in velocity space, could be discerned in the $\mathrm{Na}$ I profiles. For a small number of sightlines we also detect Ca I at $4226.73 \AA$. Equivalent widths, heliocentric radial velocities, and profiles for $\mathrm{K} \mathrm{I}$ and $\mathrm{Ca} \mathrm{I}$ are included in Tables 2 and 5, and Fig. D.1, respectively. Equivalent widths and profiles are not provided for the highly saturated $\mathrm{Na}$ I doublet as these preclude any column density measurements. However, approximate central velocities are included in Table 5. Similarly, the Ca II line is saturated, but also suffers from stellar contamination and reduced spectral quality in the blue.

\section{Results and discussion}

In the following we present and discuss the relation between equivalent widths of the observed DIBs, molecules, and the line-of-sight reddening. We investigate whether the correlations we found can be explained in terms of the skin effect and explore the spatial variation of DIB strength and strength ratios. Furthermore, we discuss the velocity structure of the ISM, as well as a model of the dust sheet and the inferred effective interstellar radiation field (ISRF). The ISRF strength, $I_{\mathrm{UV}}$, and molecular hydrogen fraction, $f_{\mathrm{H}_{2}}$, are both discussed in view of $\sigma$ and $\zeta$-type clouds.

\subsection{DIBs and dust}

In Fig. 7 we show $W_{\lambda}(5780)$ (top) and $W_{\lambda}(5797)$ (bottom) against $E_{(B-V)}$. Both the average Galactic and Upp Sco relationships are shown. Several conclusions can be drawn immediately from this initial result: 1) a linear model does not adequately describe the relation between the measured values $\left(\chi^{2} \gg 1\right)$; 2) the average DIB strength per unit reddening in Upp Sco is similar to the Galactic average; 3 ) there is a positive trend between the amount of DIB carriers and the amount of dust in the diffuse ISM; 4) there is a significant scatter from this mean linear relationship (which is also observed for the Galaxy-wide surveys), especially for the $\lambda 5780 \mathrm{DIB}$. This results in a poor $\left.\chi_{\text {red }}^{2}>5\right)$ In particular, for sightlines with $E_{(B-V)} \approx 0.2$ to $0.3 \mathrm{mag}$ (which would typically be expected to be single diffuse cloud sightlines) there is marked range in strength of the DIBs (for both $\lambda \lambda 5780$ and 5797 DIBs the strength can vary by factor of about four to five). The scatter (standard deviation) around the mean is equally high for higher $E_{(B-V)}$, but for those multiple cloud components are more likely to contribute and confuse the true variations in individual clouds. The strength-reddening relations for the $\lambda \lambda 6196,6379$, and 6613 DIBs are similar to that for $\lambda 5797$, albeit with different slopes and an increased scatter (see Fig. 8). The linear fit method using uncertainties in both parameters is an implementation of the routine FITEXY from Numerical Recipes (Press et al. 1992) where $\chi_{\text {red }}^{2}=\chi^{2} /(N-2)$, with $N$ the number of data points. A good fit will have $\chi_{\text {red }}^{2} \approx 1$. Despite significant intrinsic scatter in the DIB versus reddening relations the
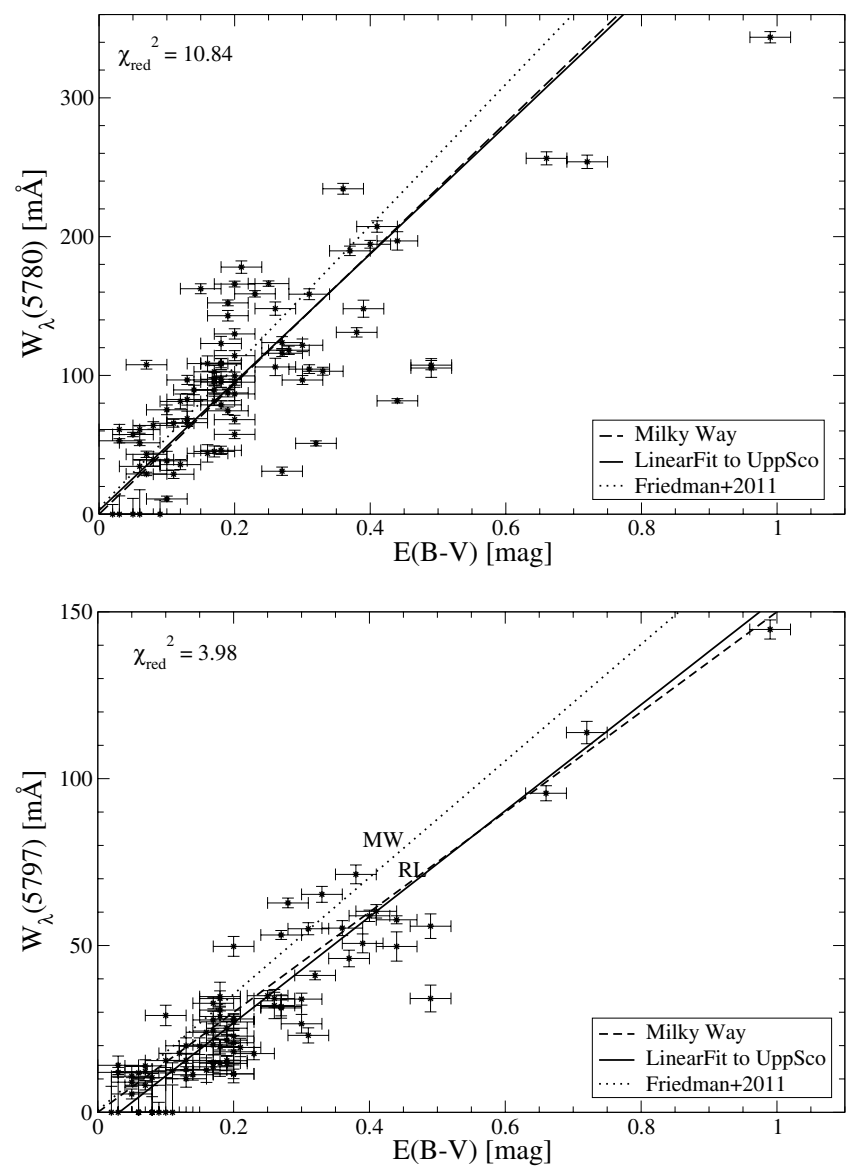

Fig. 7. Equivalent width versus $E_{(B-V)}$ for the 5780 (top) and $5797 \AA$ (bottom) DIBs. The average Galactic relationships (dashed: Cox et al. 2005; and dotted: Friedman et al. 2011) and the linear least-squares fit for Upp Sco (solid; Sect. 5) are shown. Reduced chi-squared values $\left(\chi_{\text {red }}^{2}\right)$ for the latter are indicated in the respective panels. Intercepts and slopes for the linear least-squares fits are given in Table 4. To avoid biases, the regressions were not forced to go through the origin, and upper limits for the Upp Sco data were not taken into account.

least-square linear fit results are given in Table 4 to facilitate estimates of interstellar line-of-sight reddening from observed band strengths.

These deviations could reveal the effects of local conditions on the balance between DIB carrier formation and destruction (including changes in e.g. ionisation and hydrogenation state), and therefore the abundance and physical properties of the DIB carrier. The generally positive correlation between DIB carriers and reddening suggests a link between the presence of dust grains and the molecules responsible for the diffuse bands. Figures C.1 and C.2 illustrate that there are large variations, particularly at intermediate $E_{(B-V)} \approx 0.2-0.3 \mathrm{mag}$, in the DIB strengths normalised by the amount of dust in the sightline. At lower $E_{(B-V)}$ the measurements are inaccurate, and at higher $E_{(B-V)}$ the presence of multiple clouds in the line-of-sight appears to reduce the effect of variations in individual clouds on the composite, total line-of-sight DIB spectrum. The behaviour of the DIBs in relation to molecular tracers and the local environmental conditions will be discussed in the next sections. The different behaviour of the $\lambda \lambda 5780$ and 5797 DIBs is used as a tool to study the deviations of both DIBs from the mean trend with $E_{(B-V)}$. 

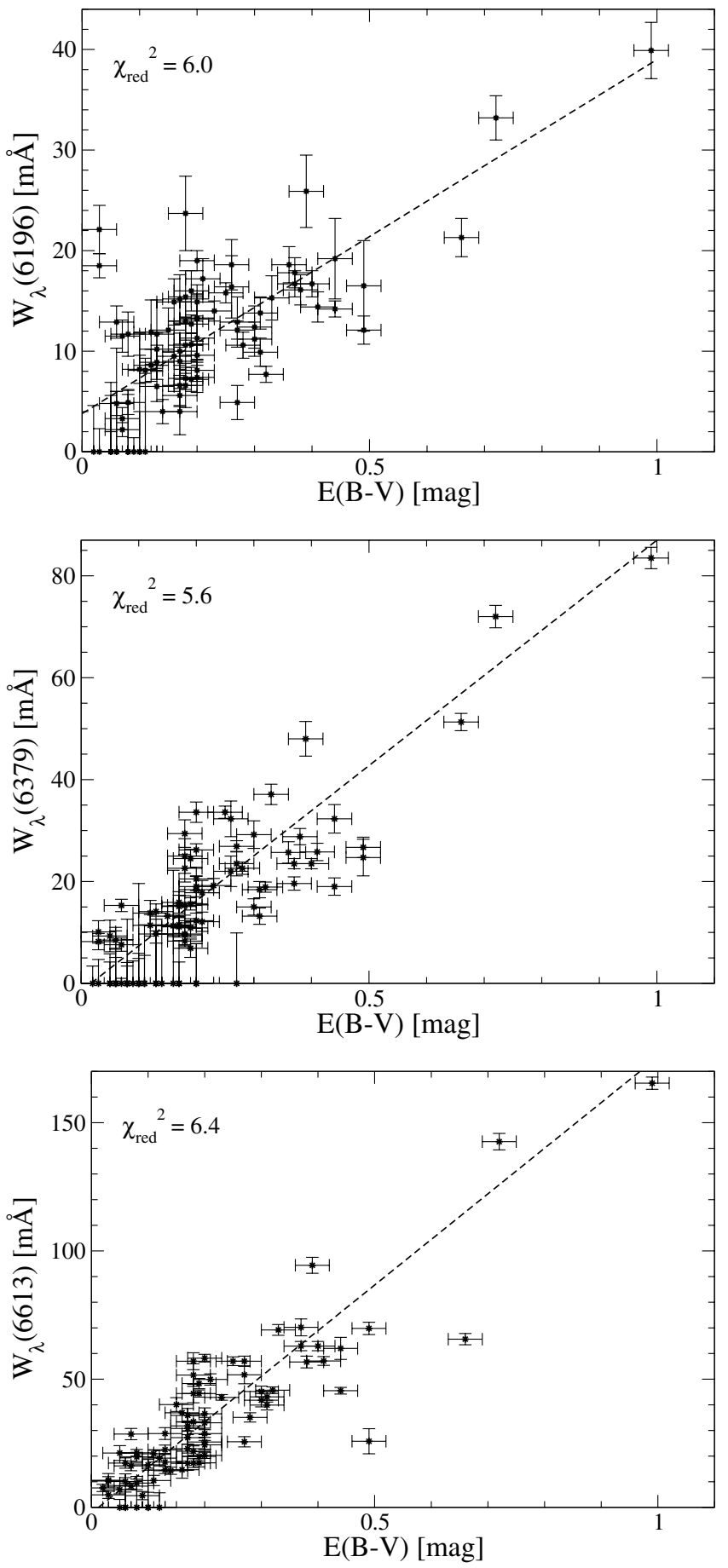

Fig. 8. Equivalent width versus $E_{(B-V)}$ for the 6196, 6379, and $6613 \AA$ DIBs observed towards the Upp Sco lines-of-sight. The reduced chi-squared $\left(\chi_{\text {red }}^{2}\right)$ for the linear fits (dashed lines) are indicated in the respective panels. The linear fit parameters are given in Table 4.

\subsection{The skin effect}

DIB carriers seem to reflect the evolutionary cycle of molecular carbon species (such as aromatic molecules) through formation, ionisation, recombination, and destruction (Cami et al. 1997; Ruiterkamp et al. 2005).

Uncharged aromatic molecules exhibit strong absorption bands in the UV and visible (blue) range while their cations and anions show specific transitions in the visible (green-yellow) and
Table 4. Slopes and intercepts of the linear least-square fits.

\begin{tabular}{lcccc}
\hline \hline Correlated parameters & $\begin{array}{c}\text { Intercept } \\
(\mathrm{m} \AA)\end{array}$ & $\begin{array}{c}\text { Slope } \\
\left(\mathrm{m} \AA / E_{(B-V)}\right)\end{array}$ & $\chi_{\text {red }}^{2}$ & $\mathrm{r}$ \\
\hline$W(5780)-E_{B-V}$ & $2.7 \pm 3.3$ & $462.0 \pm 12.7$ & 10.8 & 0.79 \\
$W(5797)-E_{B-V}$ & $-5.0 \pm 1.1$ & $159.0 \pm 4.1$ & 4.0 & 0.92 \\
$W(6196)-E_{B-V}$ & $3.8 \pm 0.4$ & $35.2 \pm 1.8$ & 6.0 & 0.72 \\
$W(6379)-E_{B-V}$ & $-1.5 \pm 0.8$ & $88.5 \pm 0.8$ & 5.6 & 0.85 \\
$W(6613)-E_{B-V}$ & $-2.2 \pm 1.2$ & $177.8 \pm 4.6$ & 6.4 & 0.86 \\
\hline$W(5780)_{\sigma}-E_{B-V}$ & $3.0 \pm 7.8$ & $640.2 \pm 43.6$ & 3.3 & \\
$W(5780)_{\zeta}-E_{B-V}$ & $-23.8 \pm 5.0$ & $419.2 \pm 13.7$ & 9.3 & \\
$W(5797)_{\sigma}-E_{B-V}$ & $-1.7 \pm 1.8$ & $127.0 \pm 9.6$ & 2.8 & \\
$W(5797)_{\zeta}-E_{B-V}$ & $-0.5 \pm 1.9$ & $153.3 \pm 5.3$ & 5.1 & \\
\hline
\end{tabular}

Notes. Uncertainties in both coordinates are taken into account. Nondetections and upper limits were excluded from the fit procedure. The fits were not forced to go through the origin, though it can be noted that in most cases the derived intercept is within $2 \sigma$ of the origin. These relations can be used to derive estimates for the (interstellar) line-ofsight reddening from measurements of the diffuse band strengths.

near-infrared (Salama et al. 1999). Each DIB carrier is thus influenced by the interstellar radiation field in a particular way, since its molecular properties such as ionisation potential and electron affinity are unique.

Interstellar clouds are exposed to the interstellar radiation field which drives their photochemistry (Snow \& McCall 2006). The UV radiation is attenuated (by dust) increasingly from cloud edge to core, giving different steady-state solutions for the photochemical reactions (like the ionisation-state) in different parts of the diffuse cloud. Thus interstellar species are subjected to stronger radiation at the edge than in the centre of the cloud.

Especially the (molecular) DIB carriers are believed to be sensitive to UV radiation. The signatures of more stable DIB carriers (such as corresponding to the $\lambda 5780 \mathrm{DIB}$ ) show a relative higher intensity in lower density, higher $I_{\mathrm{UV}}$ regions with respect to less stable DIB carriers which are more rapidly destroyed at high $I_{\mathrm{UV}}$ (e.g. $\left.15797 \mathrm{DIB}\right)$. These reach higher intensity only in more UV protected denser regions (where more stable DIB carriers like the $\lambda 5780 \mathrm{DIB}$ are less efficiently ionized and thus reduced in strength). For a sightline probing Upper Scorpius a larger amount of dust is expected to correlate with, on average, higher densities, especially as there often is only one apparent strong interstellar velocity component.

The effect of shielding (to a certain degree) of molecules from strong UV radiation is often referred to as the skin-effect (e.g. Krełowski \& Westerlund 1988; Herbig 1995). The skineffect reflects the life cycle and charge distribution of DIB carriers, which can can lead to an interpretation of high DIB carrier concentrations in the outer cloud layers. However, DIB carriers are also expected to be present in high concentrations in denser regions although in a different charge state (neutral) that can only be observed in the UV.

Cami et al. (1997) inferred that the $\lambda 5780$ DIB carrier reaches its maximum abundance when exposed to the interstellar UV radiation field (typically near the edge of a cloud), whereas the $\lambda 5797$ DIB carrier is more easily ionised and destroyed. Even more, at very low $E_{(B-V)}(<0.1 \mathrm{mag})$ only very few DIB carriers survive due to the high rate of UV photons (Jenniskens et al. 1994). The relative abundance between the $\lambda \lambda 5780$ and 5797 DIBs reflects an interplay between neutral, ionised, and destroyed DIB carriers along the entire line of sight. This balance is affected not only by the impinging radiation field, but also by 
the carbon abundance and the dust particle size distribution (Cox \& Spaans 2006). A difference in the observed ratio of these two DIBs is thus directly related to the skin-effect.

Krelowski (1989); Sneden et al. (1991); Krełowski et al. (1992) identified two types of clouds, referred to as $\sigma$ and $\zeta$-type. $\sigma$-type clouds show atomic lines and DIBs, but the molecular lines are weak or absent, while $\zeta$-type clouds have strong diatomic lines in addition to DIBs. The main difference between both types lies in a combination of density and UV irradiation by the ISRF, with $\sigma$ clouds associated with low density and/or strong exposure to UV radiation, while $\zeta$ clouds are associated with higher densities and/or more protection from UV radiation. Therefore, differentiation between $\sigma$ and $\zeta$-type clouds is directly linked to the skin-effect described previously. For sightlines probing $\zeta$-type clouds the $5797 \AA$ DIB is deeper than the $5780 \AA$, while for $\sigma$-type clouds the reverse is observed. Therefore, the $W(5797) / W(5780)$ ratio has been used to distinguish between UV exposed $(\sigma)$ and UV protected $(\zeta)$ sightlines. The nomenclature for the $\sigma$ and $\zeta$ type sightlines is historical and based on the representative lines-of-sight towards $\sigma$ Sco and $\zeta$ Oph, respectively (Krełowski \& Westerlund 1988; Krełowski et al. 1992; Krełowski \& Sneden 1995). Note that both sightlines are included in our analysis.

In this work we re-establish this classification, assuming a relatively equal distribution of sightlines probing dense versus diffuse clouds. Sightlines are classified $\sigma$ when the ratio is lower than the weighted mean of the ratio minus $1 \sigma$, while ratios higher than the weighted mean plus $1 \sigma$ are classified as $\zeta$. The remaining sightlines are classified as intermediate. The results of this selection for individual lines-of-sight are included in Table 2. Figure 9 shows that the application of our classification to the data in Fig. 7 improves the relation (reduced scatter) between DIB strength and reddening. Indeed, Fig. 9 shows that $W(5780)-E_{(B-V)}$ has an improved reduced $\chi^{2}$ for the $\sigma$ and $\zeta$ sightlines respectively, though only a marginal improvement is found for $W(5797)-E_{(B-V)}$ (where higher $W(5797)$ tend to correspond to $\zeta$-type environments) revealing the $\lambda 5780 \mathrm{DIB}$ is primarily giving rise to variations in the $W(5797) / W(5780)$ ratio.

Note that the original classification is based on the central depth, $A$, of the two DIBs. If $A_{5797}>A_{5780}$ (corresponding to $W(5797) / W(5780) \gtrsim 0.4)$ the line-of-sight is considered as $\zeta$-type. Increasing our selection threshold for the DIB ratio to 0.4 would imply that the "intermediate" sources would be included in the $\sigma$-group as well as a few $\zeta$-types. However, sightlines with both low $\left(f_{\mathrm{H}_{2}}<0.3\right)$ and high $\left(f_{\mathrm{H}_{2}}>0.4\right)$ molecular content currently classified $\zeta$ would also be re-assigned as $\sigma$-type. We note that it is impossible to make a sharp distinction between $\sigma$ and $\zeta$-type sightlines as there is - as expected - a smooth transition of physical conditions characterising both types.

Figure 10 shows $W(5797) / W(5780)$ as a function of reddening. The distribution peaks at an $E_{(B-V)}$ of $\sim 0.25 \mathrm{mag}$, indicating most optimal conditions for formation of the $5797 \AA$ DIB carrier (sufficient shielding), or alternatively less optimal conditions for the carrier associated to the $5780 \AA$ DIB carrier (insufficient UV photons to transform it into its ionic form and thus not absorbing at the visible wavelength). For sightlines with $E_{(B-V)}>0.4$ mag the conditions for formation of the $\lambda 5797$ DIB are sub-optimal, but still more favourable with respect to the $\lambda 5780$ DIB carrier than for $\sigma$ sightlines. At very low $E_{(B-V)}(<0.1 \mathrm{mag})$ the $\lambda 5797$ DIB carrier is underabundant (due to more efficient destruction of molecules by the stronger ISRF) with respect to the $15780 \mathrm{DIB}$ carrier. Also, the $W(5797) / W(5780)$ ratio itself displays a bimodal distribution
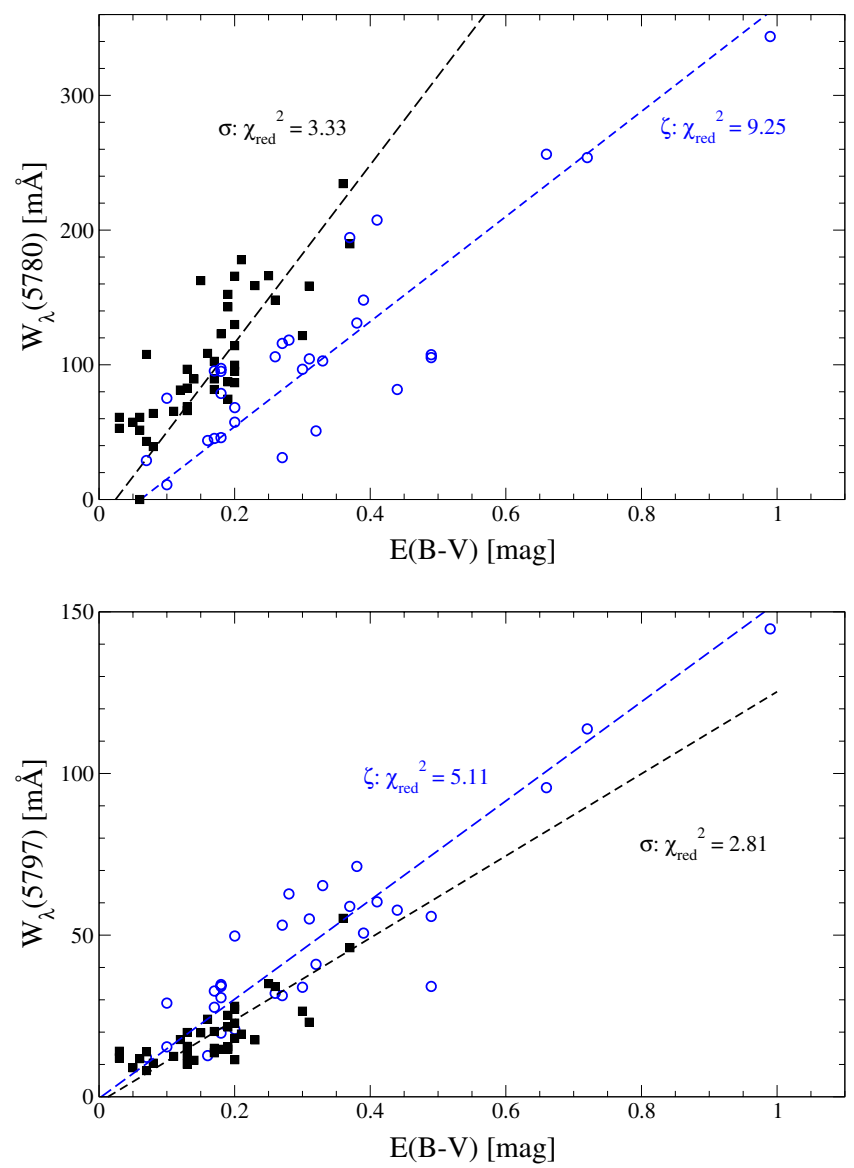

Fig. 9. $W_{\lambda}(5780)(t o p)$ and $W_{\lambda}(5797)$ (bottom) versus $E_{(B-V)}$ for the $\sigma$ and $\zeta$ subgroups, respectively. Error bars, identical to those in Fig. 7, have been omitted for clarity but taken into account for the linear fit. Parameters for the linear fit and regression are given in Table 4.

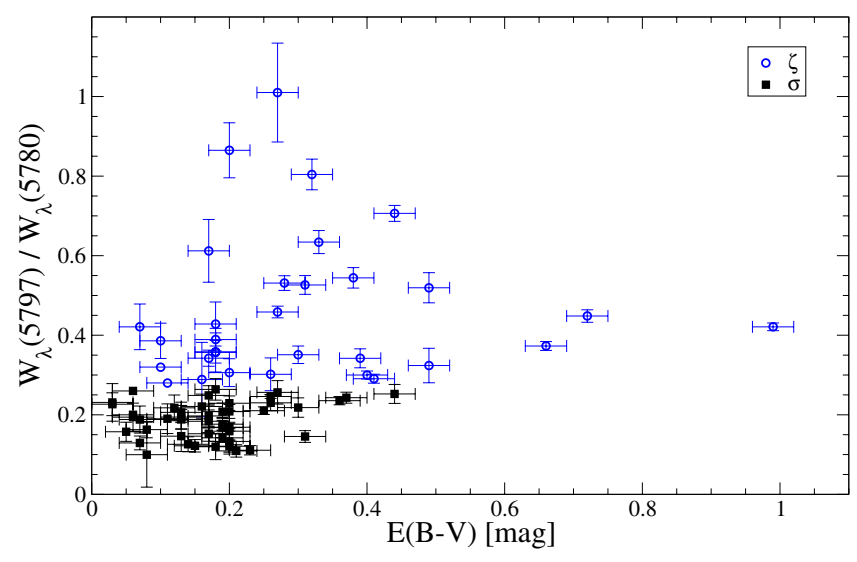

Fig. 10. The $W(5797) / W(5780)$ ratio plotted against $E_{(B-V)}$. The distribution peaks at an $E_{(B-V)}$ of $\sim 0.25 \mathrm{mag}$, indicating most optimal conditions for formation of the $5797 \AA$ DIB carrier and the destruction c.q. insufficient excitation of the molecule giving rise to the $5780 \AA$ DIB. Nonetheless, the significant scatter suggests that processes additional to dust extinction are important.

with a strong peak at about $0.2 \pm 0.05$ (Fig. 24; Sect. 5.7). The sightlines associated to this peak are predominantly $\sigma$-type (which indeed we may consider to represent typical diffuse ISM though this should be confirmed by studies of other regions). There is a second smaller peak "bump" at $\sim 0.45$, corresponding to the $\zeta$-type sightlines. Although, like the $\lambda 5797$ DIB the 

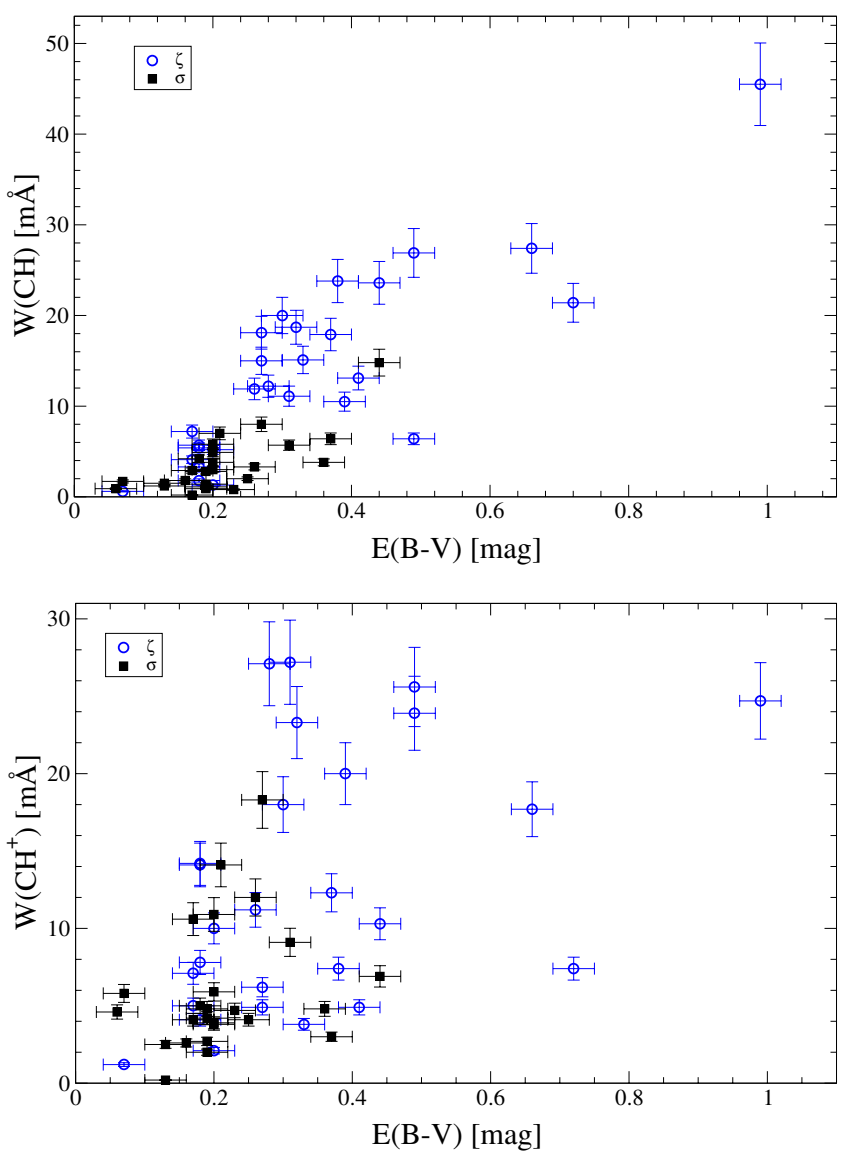

Fig. 11. Equivalent width versus $E_{(B-V)}$ for the $\mathrm{CH} 4300 \AA$ (top) and $\mathrm{CH}^{+} 4232 \AA$ (bottom) transitions. For $\sigma$ (black squares) and $\zeta$-type (blue circles) sightlines designations see Sects. 5.2 and 5.7.

$\lambda \lambda$ 6196, 6379 and 6613 DIBs also show signficant scatter on the respective $W-E_{(B-V)}$ trends, the $\lambda 5780$ DIB reveals the clearest distinction in behaviour between $\sigma$ and $\zeta$ sightlines. The link between the $W(5797) / W(5780)$ ratio and the strength of the ISRF is discussed in more detail in Sect. 5.6.

For comparison we plot also the $W(6196) / W(6613)$ ratio as a function of $E_{(B-V)}$ in Fig. C.4. This ratio is less sensitive to reddening and therefore is not such a useful tracer of local conditions such as density and UV irradiation. This is indeed expected from the recent results by McCall et al. (2010) who found an excellent correlation between the 6196 and $6613 \AA$ DIB strengths.

\subsection{Diatomic molecules and dust}

Different interstellar species are restricted to different regions (see e.g. Fig. 6 in Pan et al. 2005): $\mathrm{CN}$ and $\mathrm{CO}$ are present in dense regions, $\mathrm{CH}$ and $\mathrm{K}_{\mathrm{I}}$ are predominantly present in moderately high density regions $\left(n>30 \mathrm{~cm}^{-3}\right)$, and $\mathrm{CH}^{+}$and $\mathrm{Ca}$ I in intermediate density regions $\left(n \sim 10-300 \mathrm{~cm}^{-3}\right)$.

In Fig. 11 we show $W(\mathrm{CH})$ (top) and $W\left(\mathrm{CH}^{+}\right)$(bottom) versus $E_{(B-V)}$. It can be seen that $\mathrm{CH}$ correlates much better with $E_{(B-V)}$ than $\mathrm{CH}^{+}$, which is in line with previous observations (Crawford 1989; Krełowski et al. 1999). CH traces the dense, molecular gas and its abundance is directly proportional to $N\left(\mathrm{H}_{2}\right)$ as $N(\mathrm{CH}) / N\left(\mathrm{H}_{2}\right)=3.5 \times 10^{-8}$ (Federman 1982; Mattila 1986; Weselak et al. 2004; Sheffer et al. 2008). For 8 linesof-sight direct measurements of $N\left(\mathrm{H}_{2}\right)$ (IUE or FUSE; compiled in Friedman et al. 2011) can be compared to those derived

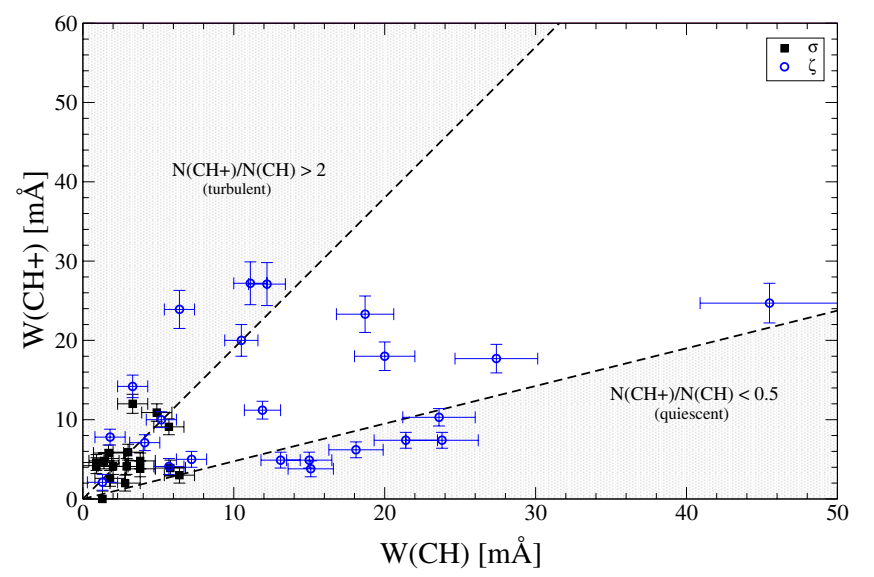

Fig. 12. $W\left(\mathrm{CH}^{+}\right)$versus $W(\mathrm{CH})$. Assuming optically thin lines the $N\left(\mathrm{CH}^{+}\right) / N(\mathrm{CH})$ ratio is equal to $0.95 \times W\left(\mathrm{CH}^{+}\right) / W(\mathrm{CH})$. Turbulent and quiescent regions are indicated by shaded areas. See text for further details. For $\sigma$ and $\zeta$-type sightlines designations see Sects. 5.2 and 5.7.

from $N(\mathrm{CH})$ in this work (Table 2). The scatter is less than $\sim 0.5$ dex, and in good agreement with the results of Sheffer et al. (2008) and references therein. Theoretically, one can thus infer the molecular hydrogen fraction $f_{\mathrm{H}_{2}}$ from $N\left(\mathrm{H}_{2}\right)$ derived from $\mathrm{CH}$ together with $N\left(\mathrm{H}_{\mathrm{I}}\right)$ derived from $W(5780)\left(\log N\left(\mathrm{H}_{\mathrm{I}}\right)=\right.$ $19.00+0.94 \log (W(5780))$; Friedman et al. 2011). The resulting values for $f_{\mathrm{H}_{2}}$ are given in Table 2. These values are consistent with - though systematically higher than - the directly measured $f_{\mathrm{H}_{2}}$ (e.g. Friedman et al. 2011; Table 2) for the eight sightlines in common. Here we have used the average Galactic relation between $W(5780)$ and $N(\mathrm{HI})$, whereas this relation may actually be lower for Upp Sco (similar to the lower gas-to-dust ratio in this region; Schlegel et al. 1998) thus leading to a higher estimate of $f_{\mathrm{H}_{2}}$. The strongly improved regression coefficient between $\mathrm{CH}$ and $E_{(B-V)}$ for the $\zeta$-type sightlines $(r=0.83)$ compared to its $\sigma$-type equivalent $(r=0.53)$ supports the interpretation that $\zeta$-type lines-of-sight trace dense gas. It is noteworthy to recall that the significant scatter for the diffuse band strengths at low $E_{(B-V)}(\sim 0.2 \mathrm{mag})$ as illustrated in Figs. 7 and 8 is not observed for $\mathrm{CH}$. $\mathrm{CH}^{+}$, on the other hand, is not a good tracer of $\mathrm{H}_{2}$ (Weselak et al. 2008a). Therefore, the low value of the correlation coefficient for $\mathrm{CH}^{+}$is not unexpected. Furthermore, note that significant amounts of $\mathrm{CH}$ are needed before $\mathrm{CN}$ is produced (Federman et al. 1984), with the latter tracing also relatively dense material (Joseph et al. 1986).

Work by Crawford (1989) suggests that the ratio of $N\left(\mathrm{CH}^{+}\right)$ and $N(\mathrm{CH})$ is indicative of the turbulent or quiescent nature of the interstellar medium in the line-of-sight. For shocked environments an offset velocity between $\mathrm{CH}$ and $\mathrm{CH}^{+}$or a velocity broadening of $\mathrm{CH}^{+}$is predicted by models. For the sightlines in this work we obtain an average $\Delta v$ of $0.3 \mathrm{~km} \mathrm{~s}^{-1}$, with individual velocity measurements that have errors of about 1 to $2 \mathrm{~km} \mathrm{~s}^{-1}$ (see also Sect. 5.5). Our data support recent surveys which find no evidence for a velocity difference between $\mathrm{CH}$ and $\mathrm{CH}^{+}$(Crane et al. 1995; Pan et al. 2005). The data do not allow for an accurate measurement and comparison of $\mathrm{CH}$ and $\mathrm{CH}^{+}$line widths. The line profiles of atomic and di-atomic species can be compared in Fig. 4 (and associated Fig. D.1). $W\left(\mathrm{CH}^{+}\right)$is plotted against $W(\mathrm{CH})$ in Fig. 12 with the turbulent $\left(N\left(\mathrm{CH}^{+}\right) / N(\mathrm{CH})>2\right)$ and quiescent $\left(N\left(\mathrm{CH}^{+}\right) / N(\mathrm{CH})<0.5\right)$ regions indicated by the shaded areas. The general correlation between $\mathrm{CH}^{+}$and $\mathrm{CH}$ (Pan et al. 2005) is poor, but it appears that two separate trends might in fact exist for the quiescent 
and turbulent regions, respectively, potentially indicative of different dominant $\mathrm{CH}^{+}$production mechanisms. The dense cloud tracer $\mathrm{CN}$ is only detected towards $\zeta$-type lines-of-sight, supporting the interpretation that the latter probe dense clouds. The $\sigma$ and $\zeta$ type sightlines show different trends for $W(\mathrm{CH})$, but not so clearly for $W\left(\mathrm{CH}^{+}\right)$.

\subsection{DIBs and small molecules}

In this section we discuss the behaviour of the $\lambda \lambda 5797$ and 5780 DIBs with respect to $\mathrm{CH}$ and $\mathrm{CH}^{+}$. Krełowski et al. (1992) found that $\mathrm{CH}$ and $\mathrm{CN}$ are only detected if the $\lambda 5797$ DIB is deeper than the $\lambda 5780$ DIB. Weselak et al. (2008b) studied $\mathrm{CH}$, $\mathrm{CH}^{+}$, and $\mathrm{CN}$ in relation to DIBs for a large, inhomogeneous sample of sightlines. These authors found a good correlation between $W(5797)$ and $N(\mathrm{CH})$, but a poor correlation between $W(\mathrm{CH}) / E_{(B-V)}$ or $W(\mathrm{CN}) / E_{(B-V)}$ versus $W(5797) / W(5780)$. The correlation of $W(5797)$ vs. $N(\mathrm{CH})$ is further improved by excluding sightlines with overabundant $\mathrm{CH}$. Their conclusion is that the $\lambda 5797$ DIB carrier is favoured in environments with higher molecular gas content. On the other hand $\mathrm{CN}$ traces a denser medium where the production of the $\lambda 5797$ DIB is apparently more inefficient.

Figures 13 and 14 show the relationship between molecular line strengths $\left(W(\mathrm{CH})\right.$ and $\left.W\left(\mathrm{CH}^{+}\right)\right)$and diffuse interstellar band strengths $\left(W_{\lambda}(5780)\right.$ and $\left.W_{\lambda}(5797)\right)$. These results are in line with Herbig (1993) and Weselak et al. (2008b), who concluded that DIB strengths correlate better with $E_{(B-V)}$ and $\mathrm{H}_{\mathrm{I}}$ than with any other feature originating from the gas phase. These DIBs have a stronger correlation with $\mathrm{CH}$ than with $\mathrm{CH}^{+}$(this work) or CN (Weselak et al. 2008b). The positive correlation with $E_{(B-V)}$ suggests that even though grains do not give rise to the diffuse bands they do play an important role in the either the DIB carrier formation - via e.g. grain surface reactions or destruction - e.g. attenuation of UV radiation - processes. The $\mathrm{CH}$ molecule and the $\lambda 5797$ DIB correlate tightly, indicating that the $\lambda 5797 \mathrm{DIB}$ carrier is most abundant in $\mathrm{CH} / \mathrm{H}_{2}$ clouds (see also Weselak et al. 2004). Some correlation is expected since both species correlate with $E_{(B-V)}$. For individual clouds a larger $W(\mathrm{CH})$ is indicative for the formation in denser clouds, which explains the tighter correlation with the $\lambda 5797$ DIB compared to the $\lambda 5780$ DIB. Note however, that the strongest molecular features potentially arise from (unresolved) multiple components of the ISM which are not necessarily denser (see e.g. velocity profiles for $\mathrm{K}_{\mathrm{I}}$ in Fig. D.1).

Figure 15 shows the $W_{\lambda}(5797) / W_{\lambda}(5780)$ ratio versus the $W(\mathrm{CH})$ (top) and $W\left(\mathrm{CH}^{+}\right)$(bottom) normalised to $E_{(B-V)}$. In agreement with Krełowski et al. (1999), a stronger correspondence is observed for the $W_{\lambda}(5797) / W_{\lambda}(5780)$ ratio versus $W(\mathrm{CH}) / E_{(B-V)}$ compared to that for $W_{\lambda}(5797) / W_{\lambda}(5780)$ versus $W\left(\mathrm{CH}^{+}\right) / E_{(B-V)}$. This confirms that $\zeta$-type clouds (dense, $\lambda 5797$ DIB favoured) are connected to a higher molecular content, implying furthermore that the DIB ratio is related to the abundance of cold cloud molecular species and properties of interstellar dust as suggested in Sect. 5.2. The poorer correlation between this DIB ratio and $W\left(\mathrm{CH}^{+}\right) / E_{(B-V)}$ then suggests that $\mathrm{CH}^{+}$forms in regions with different conditions, such as in the clouds outer edge, where the UV radiation field is much stronger.

In Fig. 16 the $W_{\lambda}(5797) / W_{\lambda}(5780)$ ratio is plotted against the $W\left(\mathrm{CH}^{+}\right) / W(\mathrm{CH})$ ratio. Again, turbulent and quiescent ISM are indicated. This plot reveals no marked correlation between these ratios. Tentatively, it shows a high DIB ratio (i.e. $\zeta$-type) for quiescent clouds and a low DIB ratio (i.e. $\sigma$-type) for turbulent clouds, which supports the idea that both $\mathrm{CH}$ and the $\lambda 5797$ DIB
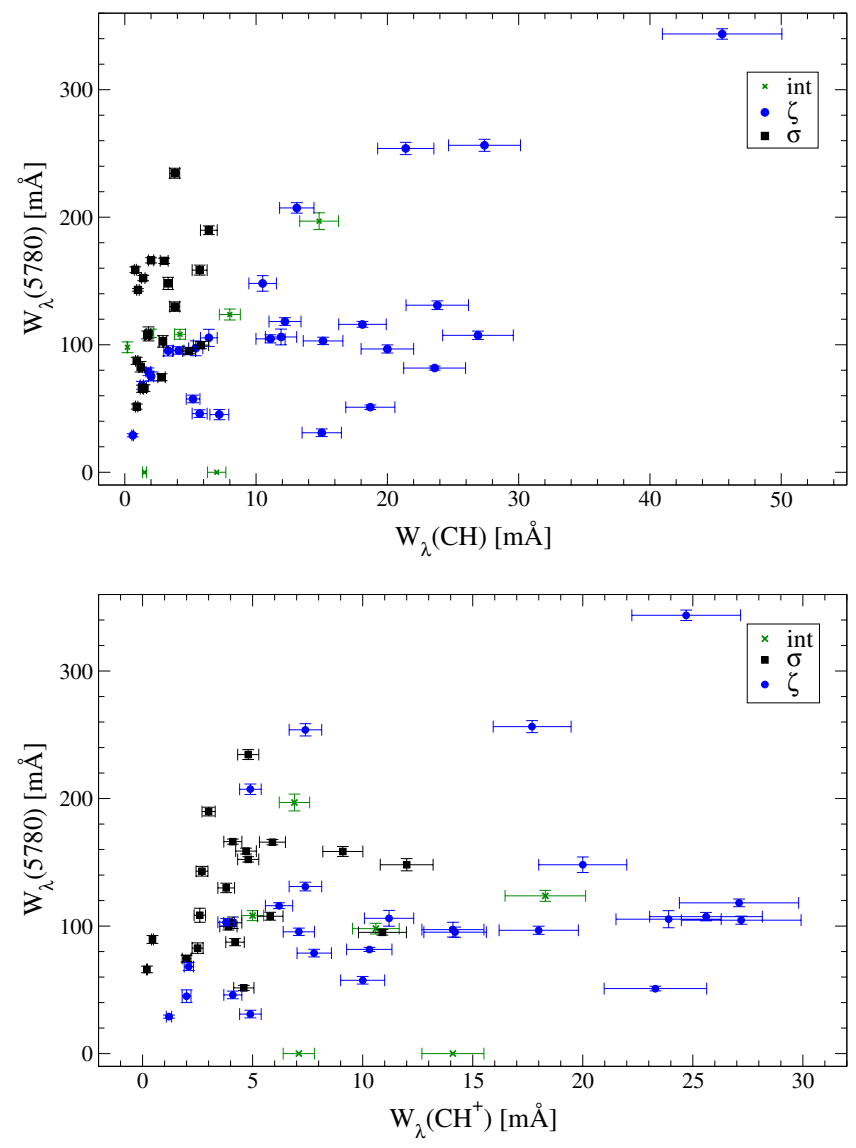

Fig. 13. $W(\mathrm{CH})$ (top) and $W\left(\mathrm{CH}^{+}\right)$(bottom) versus $W_{\lambda}(5780)$. The $\lambda 5780$ DIB shows no direct correlation with either $\mathrm{CH}$ or $\mathrm{CH}^{+}$. Looking separately at the $\sigma$ and $\zeta$-type sightlines one can distinguish different behaviour between the molecular lines and the $\lambda 5780$ DIB for both types. Sightlines classified as intermediate are indicated by green crosses. DIBs are stronger with respect to $\mathrm{CH}$ and $\mathrm{CH}^{+}$line strengths for $\sigma$-type sightlines. In other words, the $5780 \mathrm{DIB}$ carrier abundance is lower for $\zeta$-type clouds which have a higher molecular content.

trace moderately dense regions, while $\mathrm{CH}^{+}$traces the cloud edges and inter-cloud regions. Although both the $\lambda 5780$ DIB and $\mathrm{CH}^{+}$are related to the outer edges of diffuse clouds they do not reveal a strong correlation (although there appears to be a positive trend when considering only $\sigma$-type sightlines) and thus appear to react to changes in the ISRF differently. In agreement with Weselak et al. (2004) and Sect. 5.2 it seems that the Upp Sco region is somewhat turbulent, but is absent of extreme shocks. However, uncertainties in $W(\mathrm{CH})$ and $W\left(\mathrm{CH}^{+}\right)$are too large to draw firm conclusions.

\subsection{The ISM velocity distribution}

We measured the heliocentric radial velocities for both atomic and molecular lines towards Upp Sco. The strongest interstellar lines are observed at a radial velocity of about $-9 \mathrm{~km} \mathrm{~s}^{-1}$, and a weaker absorption component is detected at about $-22 \mathrm{~km} \mathrm{~s}^{-1}$ (e.g. Fig. 4). This is fully in-line with recent results reported by Snow et al. (2008) who studied 16 lines-of-sight towards the Upp Sco region. The velocity component of $-9 \mathrm{~km} \mathrm{~s}^{-1}$ corresponds to the patchy dust sheet at a distance of $110-150 \mathrm{pc}$, which is loosely connected to the $\rho$ Oph dense/molecular cloud at $122 \mathrm{pc}$ (see also Sect. 3). The other, weaker velocity 

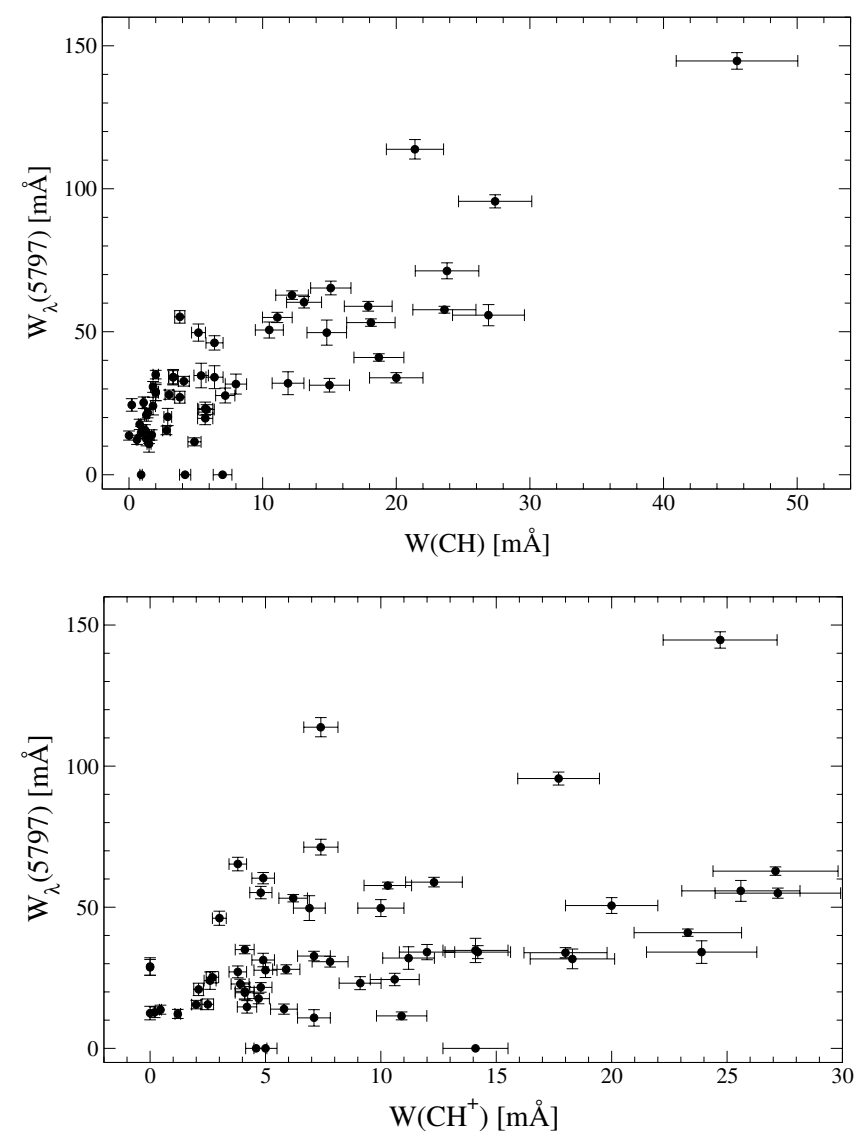

Fig. 14. $W(\mathrm{CH})($ top $)$ and $W\left(\mathrm{CH}^{+}\right)($bottom $)$versus $W_{\lambda}(5797)$. There is a moderately good correlation $(r=0.84)$ between $W(\mathrm{CH})$ and $W_{\lambda}(5797)$. There is no correlation $(r=0.47)$ between $\mathrm{CH}^{+}$and $W_{\lambda}(5797)$.

component $\left(v=-22 \mathrm{~km} \mathrm{~s}^{-1}\right)$ is linked to the tenuous low density dust layer at $\sim 50 \mathrm{pc}$. The average velocity difference between $\mathrm{CH}$ and $\mathrm{CH}^{+}$is $0.3 \mathrm{~km} \mathrm{~s}^{-1}$ (for the $\mathrm{K}_{\mathrm{I}}$ doublet lines the average velocity difference is $0.07 \mathrm{~km} \mathrm{~s}^{-1}$ ). Within the limits of the observations we confirm that there is no evidence for a $\mathrm{CH}-\mathrm{CH}^{+}$velocity offset in Upp Sco which is predicted by models for regions with strong shocks.

The relatively broad DIBs preclude a detailed radial velocity determination (for the obtained $\mathrm{S} / \mathrm{N}$, resolving power, and spectral quality). However, first order estimates (for the sightlines with strong DIBs) show no systematic differences between atomic, molecular, and DIB velocities. For the relatively narrow $5797 \AA$ DIB we measure radial velocities roughly between -20 to $0 \mathrm{~km} \mathrm{~s}^{-1}$.

This large set of radial velocity information allows us to map the velocity of the diffuse ISM clouds in front of the observed stars, very similar to the work by Snow et al. (2008). In Fig. 17 we show the color-coded interstellar radial velocity of $\mathrm{K}_{\mathrm{I}}$ and $\mathrm{Na} \mathrm{I}$ as a function of declination and right ascension. Assuming that indeed the observed sightlines probe different parts of a single dust-sheet, it thus appears that this sheet is moving differentially. The upper-left corner of the sheet (where most young OB stars are) is moving towards us while the lowerright corner remains stationary (ignoring any velocity component perpendicular to the line-of-sight). The three-dimensional kinematic motions can not be fully reconstructed with these data.
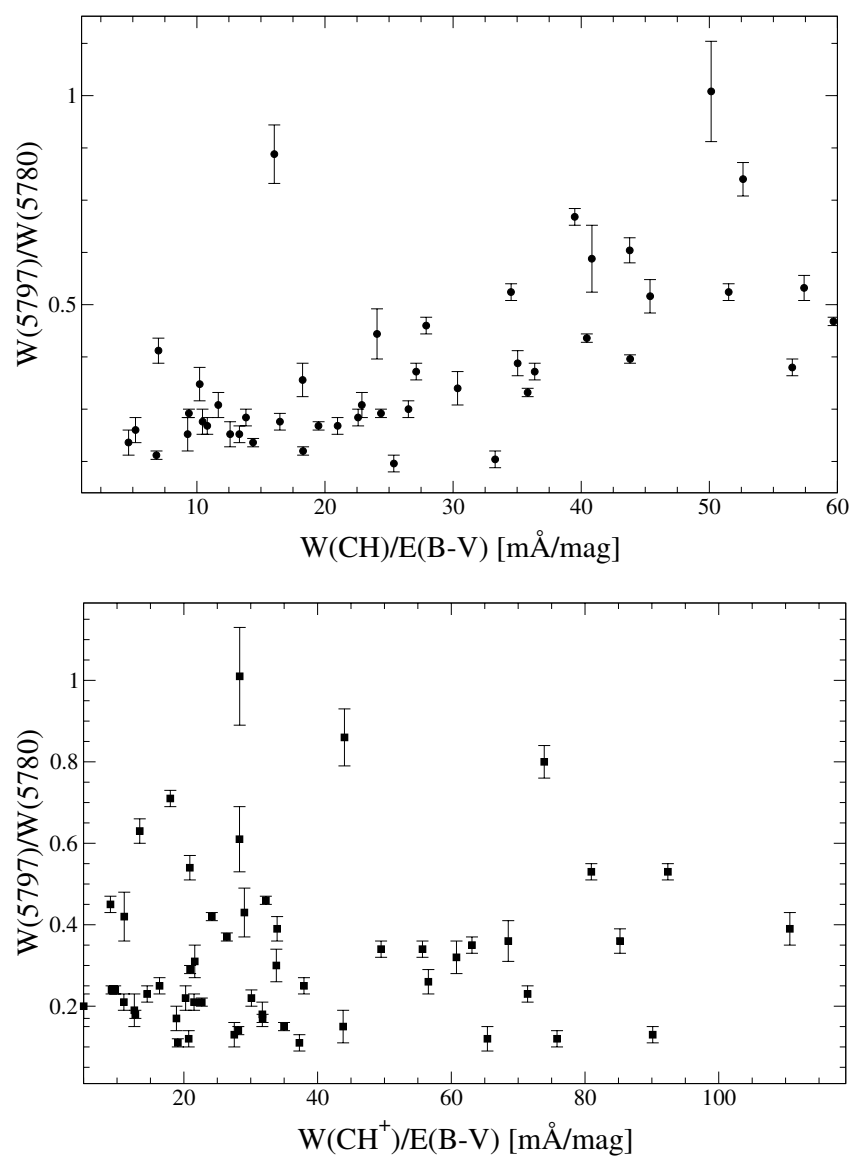

Fig. 15. $W_{\lambda}(5797) / W_{\lambda}(5780)$ against $W(\mathrm{CH}) / E_{(B-V)} \quad($ top $)$ and $W\left(\mathrm{CH}^{+}\right) / E_{(B-V)}$ (bottom). Tentatively a positive trend can be discerned between the DIB ratio and $W(\mathrm{CH}) / E_{(B-V)}(t o p)$ but not between the DIB ratio and $W\left(\mathrm{CH}^{+}\right) / E_{(B-V)}($ bottom).

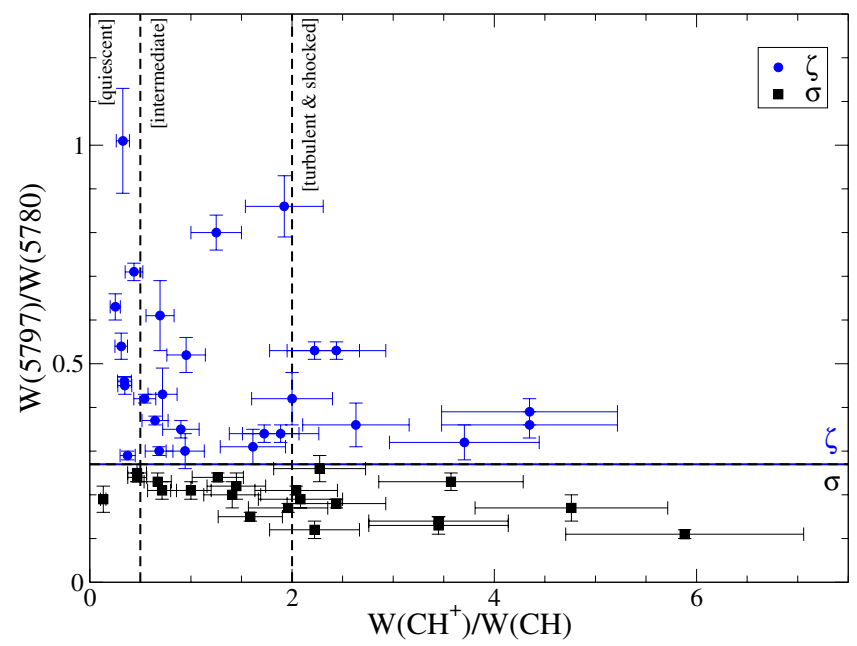

Fig. 16. $W_{\lambda}(5797) / W_{\lambda}(5780)$ vs. $W(\mathrm{CH}) / W\left(\mathrm{CH}^{+}\right)$. There is a tentative trend for decreasing DIB ratio with increasing $\mathrm{CH}^{+} / \mathrm{CH}$ ratio.

\subsection{The interstellar radiation field strength}

In order to estimate the effective interstellar radiation field for each interstellar cloud probed by the Upp Sco stars we constructed a simplified model of a sheet of dust irradiated by several OB-type stars. The thickness of the dust sheet will roughly depend on the volume and column density of H. For $n_{\mathrm{H}}=$ $100 \mathrm{~cm}^{-3}$ and $N(\mathrm{H})=5 \times 10^{21} \mathrm{~cm}^{-2}\left(E_{(B-V)} \sim 1 \mathrm{mag}\right)$ the 


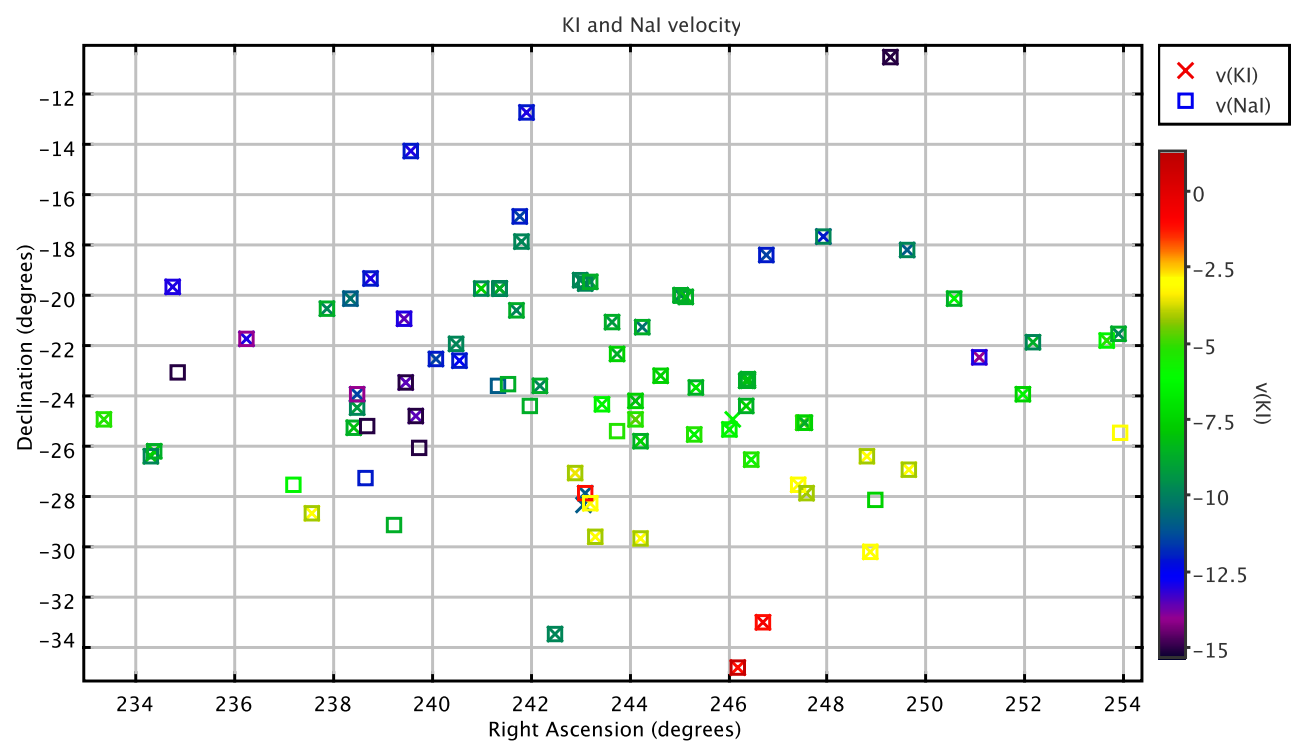

Fig. 17. The heliocentric radial peak velocity for $\mathrm{K}_{\mathrm{I}}$ and $\mathrm{Na}$ I are shown in a sky coordinate (right ascension and declination) plot. The radial velocity of the gas (in the diffuse ISM) in Upp Sco is highest in the upper-left corner, the material is approaching with velocities up to $15 \mathrm{~km} \mathrm{~s}^{-1}$ (along the line-of-sight). The gas in the lower-right corner has the lowest velocity (with respect to the Sun). (See on-line electronic version for colour figure.)

thickness is $\sim 16 \mathrm{pc}$. The dust sheet can be represented by a homogeneous thin slab at a distance of $120 \mathrm{pc}$ and a thickness of $20 \mathrm{pc}$. In this way, the distribution of individual clouds can be represented by a single sheet, which is a valid assumption because (1) $\tau_{\mathrm{UV}}>1$ so photons are scattered frequently enough to loose most of their directional memory and (2) the distribution of individual clouds has a surface area covering factor larger than unity. Property (1) assures that the radiation field strength $I_{\mathrm{UV}}$ is the roughly isotropic flux that impinges on the individual clouds making up the sheet. Aspect (2) assures that each line of sight through the representative sheet has approximately the same total extinction, relevant for the attenuation of $I_{\mathrm{UV}}$. This ensures that the radiative transfer problem to be solved is that for a slab geometry. As eight OB stars contribute over $90 \%$ of the ISRF in this region these are included as the only source of the ionising radiation (Sujatha et al. 2005). These stars illuminate the interstellar cloud from behind. In this particular model one star, HD 143275 (B0.3IV), dominates the effective ISRF, even while $\zeta$ Oph (HD 149757) has the earliest spectral type.

The radiative transfer model (Spaans 1995; Spaans 1996) takes into account both absorption and scattering. The effective optical depth $\tau_{\mathrm{V}}$ is computed from the observed $E_{(B-V)}$ and subsequently $\mathrm{e}^{-\tau_{v}}$ is multiplied by the individual stellar fluxes for an appropriate extinction curve for standard Milky Way dust (with $R_{\mathrm{V}}$ taken either as 3.1 or 4 ). The latter case is also considered since the Upp Sco region contains sightlines with high $R_{\mathrm{V}}$ values for the dust extinction (see Sect. 5.8). In addition, this method is also applied to compute the amount of back-scattered radiation.

For a sheet geometry, it is possible to express $I_{\mathrm{UV}}$ (in units of the Draine field) as a function of $R_{\mathrm{V}}$ and cloud position $R$.

$$
\begin{aligned}
I_{\mathrm{UV}}= & 4.7[(R+a) / a]^{2} \\
& \times \exp \left[-6.9\left(\frac{E_{(B-V)}}{0.20}\right)\left(\frac{R_{\mathrm{V}}}{3.1}\right)(20-R) \times 10^{-2}\right] .
\end{aligned}
$$

In this parametrisation of the radiative transfer grid the parameter $a$ depends on the distance to HD 143275 ( $a=3.4$ for a distance of $123 \mathrm{pc}) . E_{(B-V)}$ is the individual extinction of the cloud. The cloud position $R$ is set between 0 and $20 \mathrm{pc}(0 \mathrm{pc}$ being the sheet edge closest to the observer). Hence, $I_{\mathrm{UV}} \sim 1$ at the shielded edge, close to the mean Galactic value, and $I_{\mathrm{UV}} \sim 200$ at the bright edge. This latter value is relatively high and depends on the distance to HD 143275. Placing this star 1 pc further away results in $a=5.1$ and $I_{\mathrm{UV}}$ decreases by a factor two. With the nominal values, $I_{\mathrm{UV}} \leq 20$ for about one third of the sheet structure. Equation (1) allows a range of impinging field strengths which has subsequently been used to set up a grid of chemical models (including non-thermal production of $\mathrm{CH}$; Spaans 1995) for a given measured extinction, to determine which model clouds yield the best match to the available data. Thus effectively, for a given $E_{(B-V)}$ we extracted the $I_{\mathrm{UV}}$ reproducing best the observed $\mathrm{CH}$ and $\mathrm{CN}$, where the derived $I_{\mathrm{UV}}$ is also constrained by the observed upper limits for $\mathrm{CN}$. In this, $\mathrm{CH}^{+}$has been excluded because it is well known that canonical chemical models under produce its abundance by about two orders of magnitude. Turbulent dissipation and/or shocks are likely needed in the (endothermic) formation of $\mathrm{CH}^{+}$. To first order, $I_{\mathrm{UV}} / n_{\mathrm{H}}$ is the controlling parameter for the chemical and thermal balance. So an increase in density by a factor of 2 corresponds to an increase in $I_{\mathrm{UV}}$ by a factor of 2 . Due to the limited information available for each line-of-sight we adopted a generic density $n_{\mathrm{H}}=300 \mathrm{~cm}^{-3}$. This is representative of a cloud that is slightly denser than the ambient medium, the dust sheet, in which it is embedded. In other words, the clouds do not fill the region and the sheet is seen as a patchy complex of individual, but connected, clouds scattered in distance. Previous detailed modeling of the Upp Sco line-of-sight towards HD 147889 shows that this is likely a conservative lower limit for sightlines probing the denser parts of the $\rho$ Oph cloud. With detailed modeling, including observational constraints for additional species, Ruiterkamp et al. (2005) found a density of $1200 \mathrm{~cm}^{-3}$ and an $I_{\mathrm{UV}} \sim 10$ for this line-of-sight. Increasing the input density by a factor of four in the model above for HD 147899 would give a revised $I_{\mathrm{UV}}$ of 6, already in better agreement with the detailed analysis. Also, Zsargó \& Federman (2003) found C I densities between 100 
and $300 \mathrm{~cm}^{-3}$ for HD 143275 and HD 147165, but lower values, $\sim 50$ to $\sim 200$, for HD 144470 and HD 144217. However, as $\mathrm{C}_{\mathrm{I}}$ traces the purely atomic phase of clouds, it is likely that this yields lower densities than for the molecular/shielded parts as traced by e.g. $\mathrm{C}_{2}, \mathrm{CN}$ and $\mathrm{CH}$. Clearly, the simplifications introduced in the model presented in this work do not fully incorporate all the intricacies of a full-fledged analysis. However, the strength of this model, which relies only on the $\mathrm{CH}$ and $\mathrm{CN}$ abundance, is in giving statistically relevant predictions of the ISRF for a larger dataset for which only limited information is available. For accurate equivalent width measurements of both $\mathrm{CH}$ and $\mathrm{CN}$ the computed $I_{\mathrm{UV}}$ has an uncertainty of approximately $25 \%$, not including any unknown systematic effects. Uncertainties in the density, $D=\mathrm{d} n / n$, propagate into $I_{\mathrm{UV}}$ as $D^{1 / 2}$. If only $\mathrm{CH}$ is detected the value of $I_{\mathrm{UV}}$ should therefore be considered indicative only (like a model dependent lower limit). The resulting interstellar radiation field strengths are presented in Table 2.

For diffuse clouds the ISRF can also be estimated from steady-state gas phase chemistry (see e.g. Welty et al. 2006; Ritchey et al. 2006):

$I_{\mathrm{UV}} / n_{\mathrm{H}} \propto \frac{N\left(\mathrm{CH}^{+}\right)}{N(\mathrm{CH})} f_{\mathrm{H}_{2}}$

which is valid for non-thermal $\mathrm{CH}$ production and for small values of $f_{\mathrm{H}_{2}}$. On the other hand, rotational excitation modeling of $\mathrm{H}_{2}$ gives (see e.g. Jura 1975; Black \& van Dishoeck 1987; Lee et al. 2007):

$\log \left(n_{\mathrm{H}} / I_{\mathrm{UV}}\right) \propto \log f$

which is appropriate for $n\left(\mathrm{H}_{2}\right) \ll n(\mathrm{H}) \approx n_{\mathrm{H}}$ (but the linearity holds also for higher $N\left(\mathrm{H}_{2}\right)$ (e.g. Lee et al. 2007). Note that Eqs. (2) and (3) show an opposite dependence of $I_{\mathrm{UV}} / n_{\mathrm{H}}$ on $f_{\mathrm{H}_{2}}$.

In addition, $\mathrm{UV}$ pumping can produce excited $\mathrm{H}_{2}^{*}$ leading to an enhancement in the production of $\mathrm{CH}^{+}$via $\mathrm{C}^{+}+\mathrm{H}_{2}^{*} \rightarrow$ $\mathrm{CH}^{+}+\mathrm{H}$. Therefore, we compare the independently obtained values for $\mathrm{CH}^{+}$and $I_{\mathrm{UV}}$, as well as $\mathrm{CH} / \mathrm{CH}^{+}$and $I_{\mathrm{UV}}$ to investigate whether this process is important. Figure 18 illustrates that sightlines with high $\mathrm{CH}^{+}$abundances show only moderate values for $I_{\mathrm{UV}}$ (i.e. less than 10), while sightlines with high $I_{\mathrm{UV}}$ (i.e. larger than $\sim 10$ ) all show low-to-normal $\mathrm{CH}^{+}$abundances. From this relation it appears that a strong ISRF $\left(I_{\mathrm{UV}}>10\right)$ does not lead to enhanced $\mathrm{CH}^{+}$production, possibly because the molecular hydrogen abundance of these sightlines is too low. However, $W\left(\mathrm{CH}^{+}\right) / E_{(B-V)}$ peaks at $I_{\mathrm{UV}}=5$ which may reveal a delicate balance for the presence of UV pumping at intermediate $I_{\mathrm{UV}}$ and moderate $f_{\mathrm{H}_{2}}$. On the other hand, Fig. 19 shows that the $\mathrm{CH} / \mathrm{CH}^{+}$ratio drops rapidly for $I_{\mathrm{UV}}>4$. Thus despite a lower total $\mathrm{CH}^{+}$abundance (per unit reddening) for higher values of $I_{\mathrm{UV}}$ the relative production of $\mathrm{CH}^{+}$with respect to $\mathrm{CH}$ increases. This could be due to more efficient production of $\mathrm{CH}^{+}$or less efficient formation of $\mathrm{CH}$ in these low density, strongly $\mathrm{UV}$ exposed environments. The latter is indeed expected as $N(\mathrm{CH})$ correlates with $N\left(\mathrm{H}_{2}\right)$ whose relative presence reduces also with increasing $I_{\mathrm{UV}}$ (see below). UV pumping may thus contribute significantly to $\mathrm{CH}^{+}$formation only in diffuse clouds with sufficient abundance of both $\mathrm{H}_{2}$ and UV photons. Other mechanisms, like turbulent dissipation of mechanical energy, could also be important for $\mathrm{CH}^{+}$formation in this region.

Figure 20 (top panel) reveals an evident inverse relation between the strength of the ISRF, $I_{\mathrm{UV}}$, and the molecular hydrogen fraction, $f_{\mathrm{H}_{2}}$. This effect of lower $I_{\mathrm{UV}}$ for interstellar clouds with higher molecular fractions (and thus more efficient shielding of the UV radiation) is expected from Eq. (3). This figure

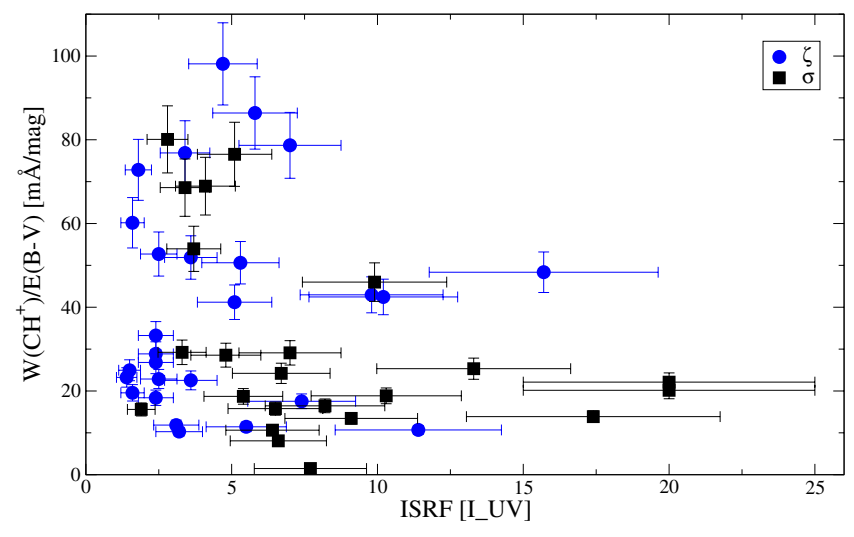

Fig. 18. The $\mathrm{CH}^{+}$line strength per unit reddening is shown as a function of the ISRF strength, $I_{\mathrm{UV}}$. Highest values for $I_{\mathrm{UV}}$ are found for low $\mathrm{CH}^{+}$abundances per unit reddening. Because $N(\mathrm{CH}) \propto N\left(\mathrm{H}_{2}\right)$ and $E_{(B-V)} \propto \mathrm{H}_{\text {I }}$ Eq. (2) gives $I_{\mathrm{UV}} / n_{\mathrm{H}} \propto N\left(\mathrm{CH}^{+}\right) / E_{(B-V)}$. There is some evidence for enhanced $\mathrm{CH}^{+}$production (UV pumping?) in clouds with moderate $I_{\mathrm{UV}} \sim 5$.

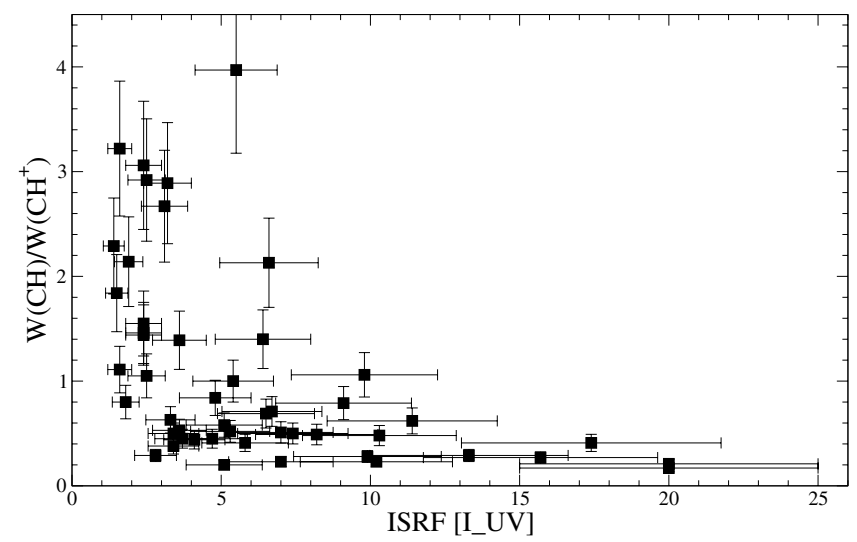

Fig. 19. The $\mathrm{CH}$ over $\mathrm{CH}^{+}$line strength ratio is plotted as a function of the ISRF strength, $I_{\mathrm{UV}}$. There is a drop in this ratio (i.e. enhanced $\mathrm{CH}^{+}$or reduced $\mathrm{CH}$ production) for stronger radiation fields $\left(I_{\mathrm{UV}}\right)$. This trend is consistent with non-thermal production of $\mathrm{CH}$, otherwise no trend would be expected.

also illustrates the general trend that the $\sigma$-type clouds have a higher $I_{\mathrm{UV}}$ and a lower molecular content $f_{\mathrm{H}_{2}}$, while $\zeta$-type sightlines have a higher molecular content and are exposed to a weaker ISRF. Note that although a few sightlines with low $f_{\mathrm{H}_{2}}$ and higher $I_{\mathrm{UV}}$ values were classified as $\zeta$-type based on the observed $W(5797) / W(5780)$ ratio, there is a clear separation based on physical conditions - between the $\sigma$ - and $\zeta$-type sightlines. Weselak et al. (2004) also show a similar distinction between $\sigma$ and $\zeta$ at $f_{\mathrm{H}_{2}} \sim 0.4$ (although their $\sigma-\zeta$ classification is based on central depth ratios resulting in a slightly different division between the two types).

The linear relation between $\log \left(f_{\mathrm{H}_{2}}\right)$ and $\log \left(n_{\mathrm{H}} / I_{\mathrm{UV}}\right)$ (i.e. Eq. (3)) in Fig. 20 (bottom panel) can be compared directly to Fig. 2 in Lee et al. (2007) showing indeed a close relation between the molecular fraction and the ratio of hydrogen density over radiation field strength, $n_{\mathrm{H}} / I_{\mathrm{UV}}$. This relation is sensitive to the total $\mathrm{H}_{2}$ column density but does not depend strongly on the hydrogen particle density, $n_{\mathrm{H}}$. Nonetheless, knowledge of the latter value (either estimated or derived from complementary data) is required to derive $I_{\mathrm{UV}}$. Non-thermal $\mathrm{H}_{2}$ excitation due to turbulence can mimic UV pumping and thus alter the relation between the model $I_{\mathrm{UV}}$ and the observed $f_{\mathrm{H}_{2}}$ (Spaans 1995). Thus, 

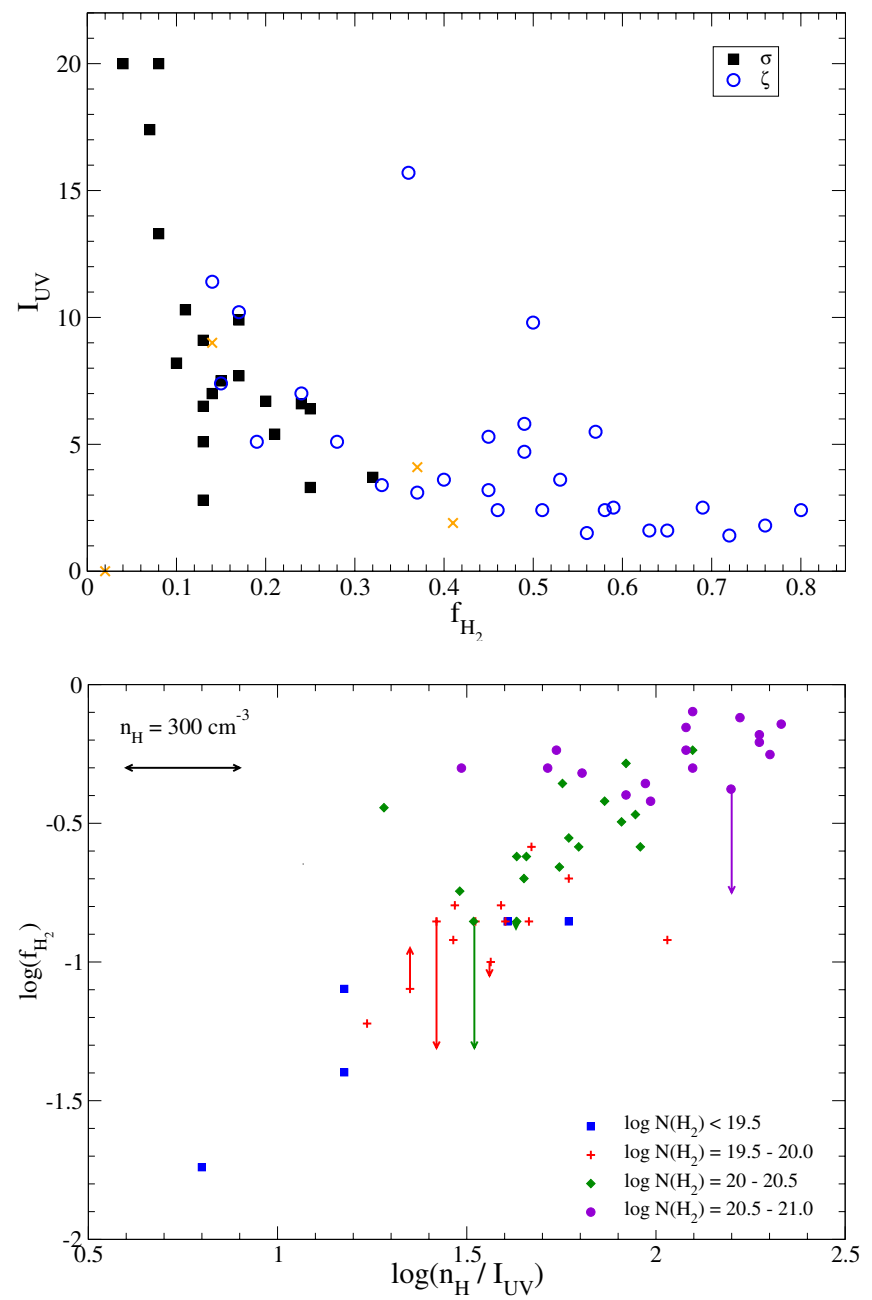

Fig. 20. (Top) the model $I_{\mathrm{UV}}$ is plotted as a function of inferred $f_{\mathrm{H}_{2}}$. (Bottom) alternatively, the molecular hydrogen fraction can be plotted as a function of the ratio of the hydrogen density of the UV radiation field strength, for our general value of $n_{\mathrm{H}}=300.0 \mathrm{~cm}^{-3}$. As expected from Black \& van Dishoeck (1987), $\log \left(f_{\mathrm{H}_{2}}\right)$ is directly proportional to $\log \left(n_{\mathrm{H}} / I_{\mathrm{UV}}\right)$, where the intercept of this relation depends on the total $\mathrm{H}_{2}$ column density (see also Fig. 2 in Lee et al. 2007). Ranges for inferred $N\left(\mathrm{H}_{2}\right)$ are indicated by different symbols. The horizontal arrow gives the change in $\log \left(n_{\mathrm{H}} / I_{\mathrm{UV}}\right)$ for an increase or decrease of $n_{\mathrm{H}}$ by a factor of 2. The vertical arrows on the data points indicate the correction of the inferred fraction to the directly observed fraction. Note that the inferred molecular fraction, $f_{\mathrm{H}_{2}}$, directly depends (non-linearly) on the ratio of $W(\mathrm{CH})$ over $W(5780)$, and the $I_{\mathrm{UV}}$ depends also on $\mathrm{CH}$, as well as $\mathrm{CN}$ and $E_{(B-V)}$.

in addition to deriving the effective ISRF strength in interstellar clouds (averaged along the line-of-sight) in Upp Sco with the model above we can use measurements of the $\lambda 5780 \mathrm{DIB}, \mathrm{CH}$ and $\mathrm{CN}$ absorption line strengths to estimate $N\left(\mathrm{H}_{2}\right), N\left(\mathrm{H}_{\mathrm{I}}\right)$, and the molecular hydrogen fraction, $f_{\mathrm{H}_{2}}$.

Figure 21 shows the dependence of the W(5797)/ $W(5780)$ ratio on the ISRF, $I_{\mathrm{UV}}$. In general, sightlines with low $I_{\mathrm{UV}}$ values are $\zeta$-type sightlines for which also $\mathrm{CN}$ has been detected. And lines-of-sight for which we find high values of $I_{\mathrm{UV}}$ have, on average, lower values for the $W(5797) / W(5780)$ ratio. The few $\zeta$-type clouds with high $I_{\mathrm{UV}}$ have in fact DIB ratios that are close to the average ratio used to discriminate between $\sigma$ and $\zeta$-type environments. On the other end, there are also a few $\sigma$-type clouds associated with a weak ISRF. We recall that a change in the density will give an equal change of $I_{\mathrm{UV}}$, which

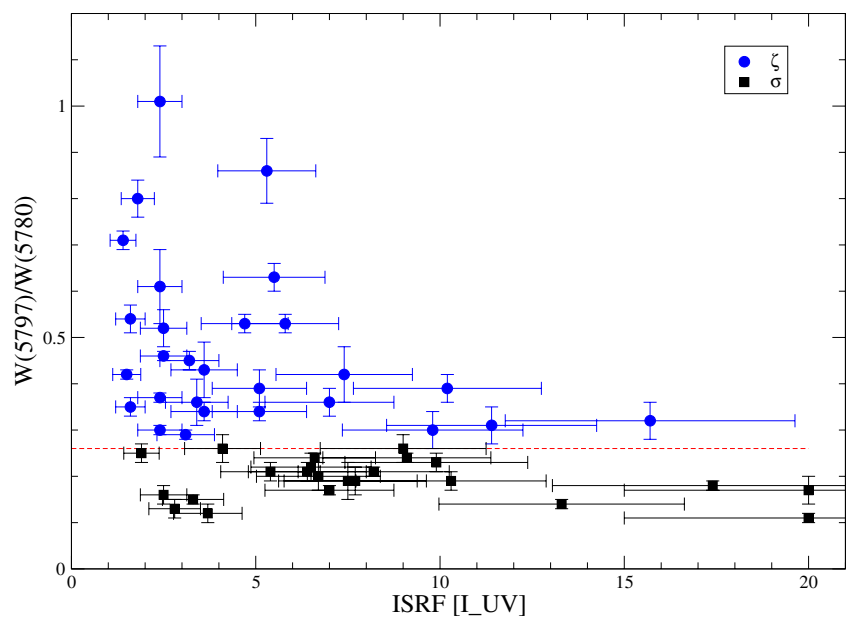

Fig. 21. The $W(5797) / W(5780)$ DIB ratio is plotted against the ISRF, $I_{\mathrm{UV}}$. High DIB ratios (i.e. $\zeta$ ) correspond to a lower $I_{\mathrm{UV}} . \sigma$-type clouds show a similar range in $I_{\mathrm{UV}}$.

could consequently shift individual sightlines to either lower or higher $I_{\mathrm{UV}}$, thus introducing additional scatter. Figure 21 is consistent with a $5797 \AA$ DIB carrier which requires sufficient protection from UV radiation in order to survive in the diffuse ISM, while conversely the $5780 \AA$ DIB carrier requires UV photons for excitation (possibly because the carrier needs to be ionised in order to absorb at $5780 \AA$ ). At this point it is important to note that the sightlines with higher inferred $I_{\mathrm{UV}}$ all rely on $\mathrm{CH}$ measurements only and should therefore be considered indicative. Also, the average $I_{\mathrm{UV}}$ values for respectively $\sigma$ and $\zeta$ type sightlines are within $1 \sigma$ of each other (where the mean of $I_{\mathrm{UV} \sigma}$ is two times the mean of $I_{\mathrm{UV} \zeta}$ ). Higher sensitivity data of CN transitions in Upp Sco are required to accurately probe $I_{\mathrm{UV}}$ throughout the region. In that case, subsequent comparisons with accurate $\mathrm{CH}^{+}$line-widths (to determine the Doppler velocity parameter $b$ ) could be used to distinguish between the production of $\mathrm{CH}^{+}$in shocks (c.q. turbulent media) and the effect of UV pumping on enhanced abundances of $\mathrm{CH}^{+}$(see also Sect. 5.3).

\subsection{Spatial distribution of DIBs and DIB ratios}

Our dataset provides a unique opportunity to investigate the scatter on the linear relation between DIB strength and reddening by dust, in particular with respect to its spatial distribution. Therefore, the equivalent width per unit reddening is plotted on the infrared dust map (Schlegel et al. 1998; Fig. 22). We show only the results for the 5780 and $5797 \AA$ DIBs. The equivalent widths for the 6196,6379 , and $6613 \AA$ DIBs behave similarly to the $5797 \AA$ DIB, but due to the larger relative uncertainties in the measured equivalent widths are not discussed further.

From Fig. 22 it is apparent that the spatial behaviour for the two DIBs is different. In order to visualize this effect we show the spatial distribution of the $W_{\lambda}(5797) / W_{\lambda}(5780)$ ratio in Fig. 23. It can be seen that $\sigma$-type sightlines are more frequently probed towards the region westward of the $\rho$ Oph cloud which has a low dust content, while predominantly $\zeta$-types are observed towards the high dust column density $\rho$ Ophiuchus cloud complex. Sujatha et al. (2005) showed that 5 of the 8 stars producing $90 \%$ of the local ISRF are situated in this low-dust region at distances between 109 and 141 pc. Here, the strong stellar winds have blown out most of the dust and are now impinging on the west side of the $\rho$ Oph cloud. 

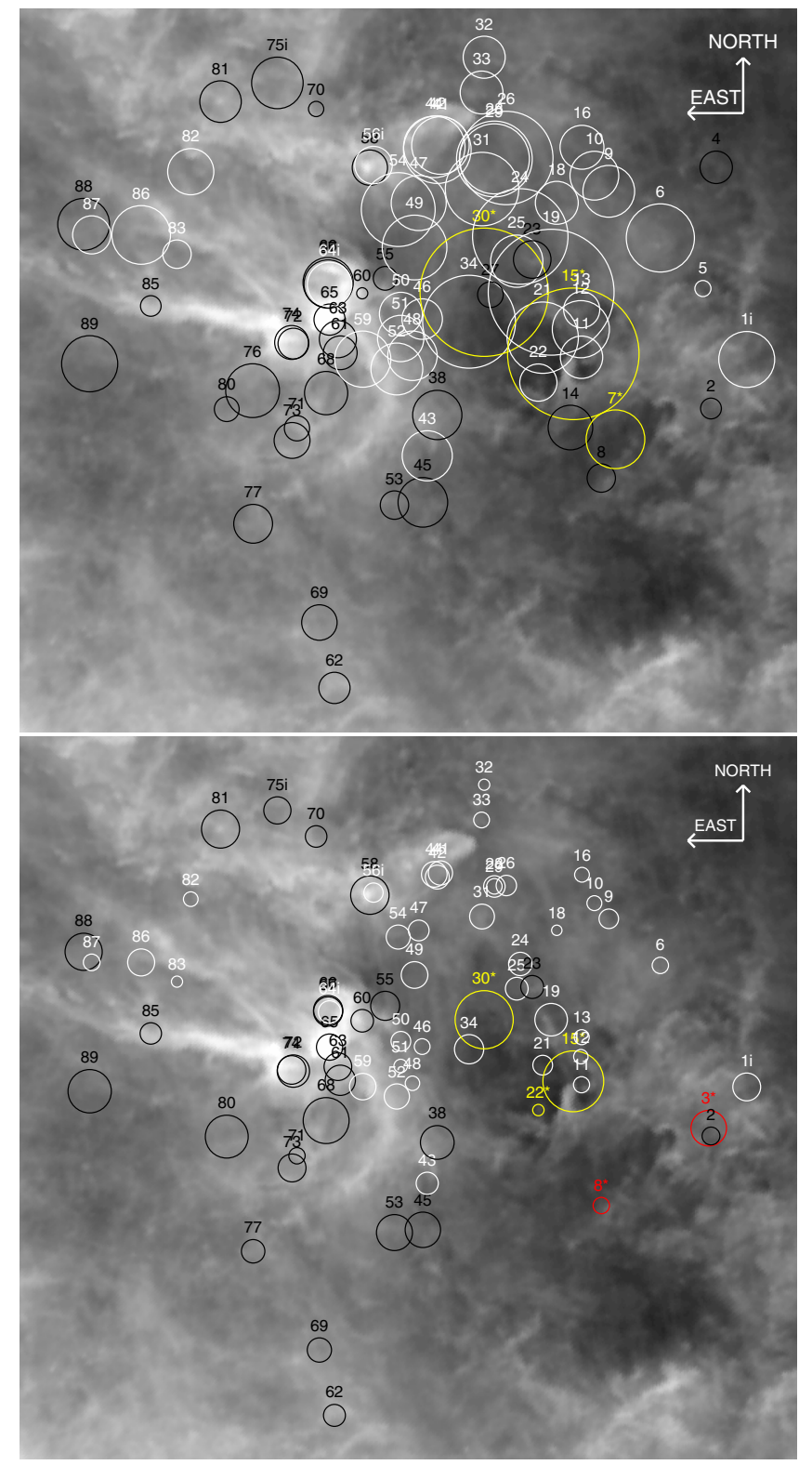

Fig. 22. Circles correspond to $W_{\lambda}(5780) / E_{(B-V)}$ (top) and $W_{\lambda}(5797) /$ $E_{(B-V)}$ (bottom). Background: IRAS $100 \mu \mathrm{m}$ dust map. Targets are indicated by their target number as defined in Table 1 . White circles represent $\sigma$-type sightlines and black circles $\zeta$-type sightlines, except those labeled " $\mathrm{i}$ " which are of intermediate type. Targets labeled with "*”" have relative errors on the ratio between 30 and $60 \%$, while errors larger than $60 \%$ are omitted. The circle sizes for $W(5797) / E_{(B-V)}$ are multiplied by a factor 2 with respect to those for $W(5780) / E_{(B-V)}$.

To study this difference we selected two regions, a region free of dust emission and one with strong dust emission, respectively. The first region is centred on the $\rho$ Oph cloud (showing a high dust column), while the second region is centred on the region west of $\rho \mathrm{Oph}$ scarce in dust emission (Fig. 23, black and white squares, respectively). In line with the observed dust density, UV field strength and molecular $\mathrm{H}_{2}$ fraction, $80 \%$ of the lines-of-sight in the selected "high-density" (i.e. higher dust column and higher $f_{\mathrm{H}_{2}}$ ) region are classified as $\zeta$ and $95 \%$ of the sightlines in the "low-density" (i.e. low dust column density and low $f_{\mathrm{H}_{2}}$ ) region are designated $\sigma$-type.

The weighted mean and the associated error of the DIB ratio, $I_{\mathrm{UV}}$, and $f_{\mathrm{H}_{2}}$ are calculated for the "low-density" and

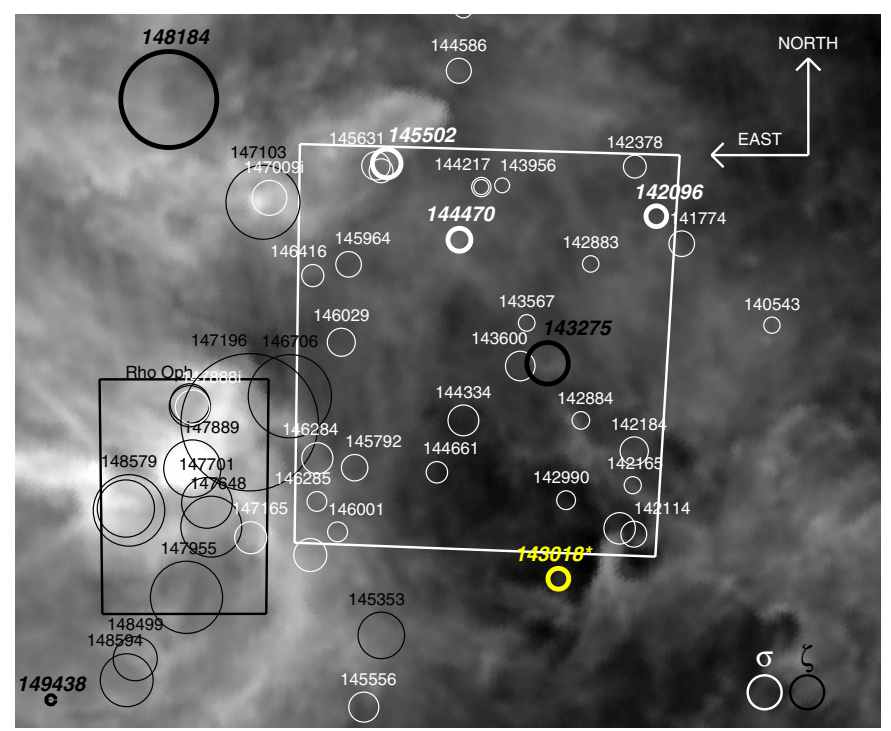

Fig. 23. The circle sizes is proportional to $W_{\lambda}(5797) / W_{\lambda}(5780)$. Black and white circles indicate $\zeta$ and $\sigma$ sightlines, respectively. Seven of the eight stars (one is outside the map) generating $90 \%$ of the local ISRF (Sujatha et al. 2005) are indicated by bold circles with italic numbers. The "high-density" ( $\rho$ Oph cloud) and "low-density" regions selected for comparison are delineated by the black and white square, respectively. Intermediate classified lines-of-sight are labeled with an "i". Note that for HD 149438 no DIBs were detected, but it is included here for its large contribution to the local ISRF. Targets labeled with "*” have relative errors on the ratio between 30 and $60 \%$, while errors larger than $60 \%$ are omitted. All other targets have errors smaller than $30 \%$ (see also Table 2).

Table 6. Weighted mean and associated error of $W(5797) / W(5780)$, $I_{\mathrm{UV}}$, and $f_{\mathrm{H}_{2}}$ for all sightlines (for which values are available) and for the "low-density" and "high-density" regions.

\begin{tabular}{lccc}
\hline \hline Weighted mean & All sightlines & "High-density" & "Low-density" \\
\hline$W(5797) / W(5780)$ & $0.26 \pm 0.01$ & $0.36 \pm 0.03$ & $0.16 \pm 0.01$ \\
$I_{\mathrm{UV}}$ & $6.4 \pm 2.5$ & $4.2 \pm 2.5$ & $8.1 \pm 6.2$ \\
$f_{\mathrm{H}_{2}}$ & $0.34 \pm 0.2$ & $0.44 \pm 0.2$ & $0.15 \pm 0.1$ \\
\hline
\end{tabular}

"high-density" selected regions, as well as for the total dataset (all sightlines with available values) and are given in Table 6. The two regions differ significantly from each other, and both regions show deviations from the overall weighted mean. For the "low-density" region the DIB ratios peak at about 0.20 and with a distribution width of about 0.05 , while the "high-density" DIB ratio distribution peaks at about 0.45 with a wider width of about 0.10 (Fig. 24). The mean value of $0.26 \pm 0.01$ for the DIB ratio has been adopted to make the distinction between $\sigma$ and $\zeta$ ) type clouds. Figure 20 shows that the mean molecular hydrogen fraction, $f_{\mathrm{H}_{2}}=0.34 \pm 0.21$ (Table 6), provides an alternate - complementary - way to distinguish between $\sigma$ and $\zeta$ sightlines (e.g. Fig. 20; top panel).

\section{8. $R_{V}$ and $W_{\lambda}(5797) / W_{\lambda}(5780)$}

For Milky Way lines-of-sight a weak relation exists between the total-to-selective visual extinction ratio, $R_{\mathrm{V}}$, and the UV extinction (Cardelli et al. 1989; Fitzpatrick \& Massa 2007). For increasing $R_{\mathrm{V}}$ the far-UV absorption decreases, suggesting that fewer small dust particles/large molecules absorb in the far$\mathrm{UV}$, and implying a dust size distribution shifted towards larger 


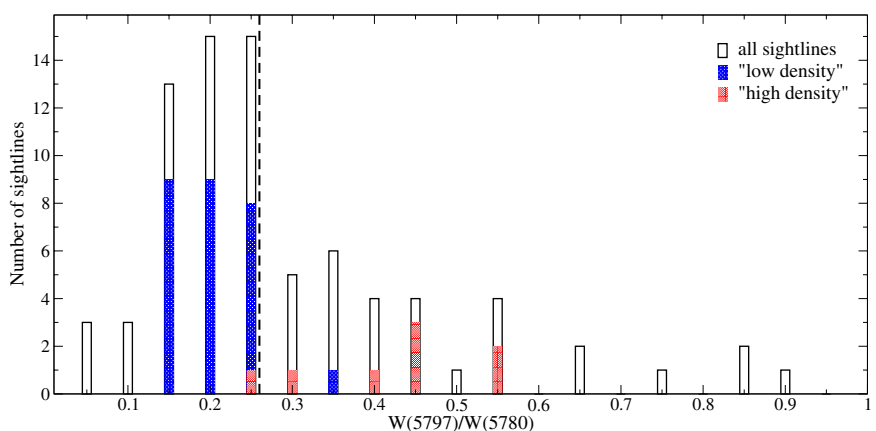

Fig. 24. Histogram of the $W(5797) / W(5780)$ ratio for the lines-of-sight in the low-density (blue) and high-density (red) region, and for all sightlines in the sample. The vertical dashed line sets the division between $\sigma$ and $\zeta$-type cloud. (See electronic version for colour version.)

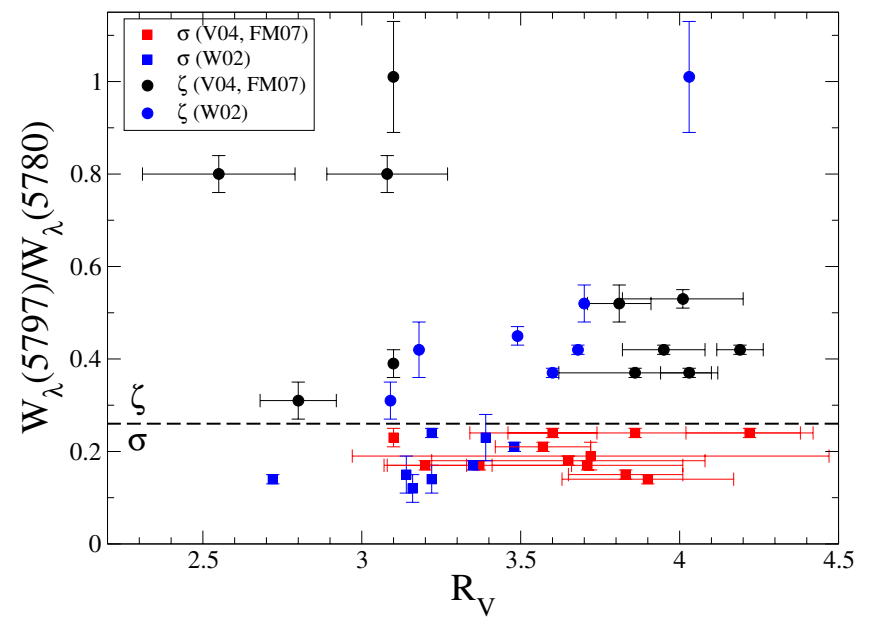

Fig. 25. $W(5797) / W(5780)$ is plotted against the total-to-selective visual extinction ratio $R_{\mathrm{V}}$ for 23 stars. The low and high density sightlines are indicated by squares and circles, respectively. Values are from Wegner (2002) [W02]; Valencic et al. (2004) [V04], and Fitzpatrick \& Massa (2007) [FM07].

grains. Therefore, $R_{\mathrm{V}}$ is sometimes used as a tracer of high density ISM, where grain growth is more significant; $R_{\mathrm{V}}=3.1$ for the average diffuse ISM, while $R_{\mathrm{V}}=4-6$ (Cardelli \& Wallerstein 1986; Cardelli 1988) for dense ISM. Whittet (1974) showed that sightlines penetrating the $\rho$ Oph cloud reveal a higher than average $R_{\mathrm{V}}$. Extinction curves with a higher $R_{\mathrm{V}}$ have less steep far-UV rise, which is associated to a lack of small particles which is often attributed to enhanced grain growth in denser clouds. However, an obvious correlation between $R_{\mathrm{V}}$ and $n_{\mathrm{H}}$, $N(\mathrm{CH}), N(\mathrm{CN})$ could not be identified. The observed differences in $R_{\mathrm{V}}$ towards Upp Sco showed that the dust size distribution is not homogeneous throughout the primordial cloud that formed the association, or that the dust has been processed differently (for example, due to destruction in UV exposed regions, or grain growth in denser areas) in the various parts of the association.

In an attempt to reveal any dependence between the UV radiation field and the dust grain size distribution we plot $W_{\lambda}(5797) / W_{\lambda}(5780)$ versus $R_{\mathrm{V}}$ for 23 targets in our sample (see Table 1). Large error bars on $R_{\mathrm{V}}$ and the large differences between values obtained by different authors prevent us from discerning any significant trends between the DIB ratio and $R_{\mathrm{V}}$. This is consistent with the earlier work of Snow \& Cohen (1974).

\section{Conclusions and summary}

Lines of sight can be designated via $W(5797) / W(5780)$ as either $\zeta$-type (sightlines penetrating cloud cores) or $\sigma$-type (sightlines probing cloud edges) (or intermediate). We investigated the spatial variation of the DIB strengths $W(\mathrm{DIB})$ and the $W(5797) / W(5780)$ DIB ratio, and their dependence on reddening in 89 lines-of-sight within a field of $20^{\circ} \times 20^{\circ}$ probing the small scale variations in the gas and dust in the Upp Sco association. This represents an in-depth multi-object study of a wellstudied interstellar cloud complex, providing a valuable statistical dataset. These data cover a wide range in dust column densities (from zero up to four magnitudes of visual extinction) to track the sensitivity of the DIB carrier molecules in relation to their local environment.

Our results provide evidence that on average the DIB strengths in Upper Scorpius are linearly proportional to the reddening, closely following the general relation observed for the Galactic diffuse ISM. In addition, we showed that the scatter on these relationships, expressed for example via the $W(5797) / W(5780)$ and $W(\mathrm{DIB}) / E_{(B-V)}$ ratios, is significant and can be attributed to variations in the local physical conditions, in particular the interstellar density and the radiation field.

We found that making a distinction between $\sigma$ and $\zeta$ type sightlines clearly improved the relation between $5780 \AA$ DIB strength and the amount of dust, $E_{(B-V)}$. The improvement for the other DIBs is less pronounced although still significant, suggesting that particularly the $5780 \AA$ DIB is sensitive to variations in the local conditions of the interstellar gas and dust.

The $\mathrm{CH}$ and $\mathrm{CH}^{+}$molecules are detected in 53 out of 89 sightlines, whereas $\mathrm{CN}$ and $\mathrm{Ca}$ I are detected in 15 and 31 sightlines, respectively. $\mathrm{CH}$ traces mainly cold (UV shielded) cloud material in the line-of-sight which is confirmed by the improved regression coefficient with respect to $E_{(B-V)}$ for $\zeta$-type sightlines $(r=0.83)$ compared to its $\sigma$-type equivalent $(r=$ $0.53)$. In line with previous results we observe no significant velocity difference between the $\mathrm{CH}$ and $\mathrm{CH}^{+}$lines in the Upp Sco region, thus finding no evidence for the production of $\mathrm{CH}^{+}$in shocks (Ritchey et al. 2011).

$\mathrm{CN}$ traces the dense cloud cores and is only detected in $\zeta$-type sightlines. The presence of (presumably very) low column densities of $\mathrm{CN}$ in $\sigma$-type sightlines remains to be confirmed.

The $W(5797) / W(5780)$ DIB ratio is more strongly correlated with $W(\mathrm{CH}) / E_{(B-V)}$ than with $W\left(\mathrm{CH}^{+}\right) / E_{(B-V)}$. This result confirms that $\zeta$-type clouds (i.e. dense and $\lambda 5797$ favoured) have a higher molecular content (e.g. higher abundances of $\mathrm{CH}$ and $\mathrm{H}_{2}$ ). The $\mathrm{CH} / \mathrm{CH}^{+}$ratio is used to discriminate between quiescent and turbulent regions, confirming the division between $\sigma$ and $\zeta$-type environments. This DIB ratio is thus related to the abundance of molecular species and dust properties inside the cloud cores.

We found a significant difference between the mean $W(5797) / W(5780)$ ratio towards the dense $\rho$ Oph cloud and towards the lower dust density region to the west. The high dust density region consists predominantly of $\zeta$-type sightlines, whereas (as expected) the low-density region contains mainly $\sigma$-type lines of sight. This distinction was used to assign all sightlines to either $\sigma$ or $\zeta$ type.

Radial velocities of atomic and molecular absorption lines reveal one main interstellar component (with possibly unresolved small-scale structure) at approximately $-9 \mathrm{~km} \mathrm{~s}^{-1}$, corresponding to the Upp Sco dust sheet at a distance of 110 to $150 \mathrm{pc}$. Another, much weaker, velocity component at $\approx-22 \mathrm{~km} \mathrm{~s}^{-1}$, is linked to the tenuous foreground dust sheet at $50 \mathrm{pc}$. 
Asymmetries and widths of the $\mathrm{Na}$ I and $\mathrm{K}$ I line profiles suggests that the main velocity component may be resolved in narrower components at higher spectral resolution. The radial velocities for individual sightlines reveal velocity gradient in the dust sheet associated to Upp Sco. This could be seen as a differentially moving sheet, with the south-west corner fixed and the north-east corner moving towards the observer. For the sightlines with the strongest DIBs, the atomic, molecular, and DIB radial velocities are similar within uncertainties, indicating that these species spatially co-exist with each other.

A simplified radiative transfer model of the main Upp Sco dust sheet was used to compute the effective interstellar radiation field (ISRF) from $\mathrm{CN}$ and $\mathrm{CH}$ observations. Five (of the eight) stars that strongly contribute to the local ISRF are located in the lower-density region of the Upp Sco cloud complex, substantiating the inferred strong radiation field. The most accurate values for $I_{\mathrm{UV}}$ are derived if both $\mathrm{CN}$ and $\mathrm{CH}$ are detected, and therefore these sightlines correspond to those probing denser $\zeta$-type regions. From the observed peak in the $\mathrm{CH}^{+} / E_{(B-V)}$ ratio for $I_{\mathrm{UV}} \approx 5$ we can infer that UV pumping plays a role in the production of $\mathrm{CH}^{+}$in the Upp Sco region for diffuse clouds with sufficiently strong UV field and significant abundance of $\mathrm{H}_{2}$. The drop in $\mathrm{CH} / \mathrm{CH}^{+}$with increasing $I_{\mathrm{UV}}$ is probably due to lower $\mathrm{CH}$ abundances for the more tenuous, UV exposed clouds. In fact the effective radiation field, $I_{\mathrm{UV}}$, is an inverse function of the molecular hydrogen fraction, $f_{\mathrm{H}_{2}}$, derived from $N(\mathrm{CH})$ and $W(5780)$. And, $\log \left(f_{\mathrm{H}_{2}}\right)$ is, as expected, proportional to $\log \left(n_{\mathrm{H}} / I_{\mathrm{UV}}\right)$. The $W(5797) / W(5780)$ ratio versus $I_{\mathrm{UV}}$ relation in Upp Sco confirms that most of the sightlines with low $I_{\mathrm{UV}}$ values are of $\zeta$-type for which also $\mathrm{CN}$ is detected, while only few $\zeta$-type sightlines have large $I_{\mathrm{UV}}$. The average $I_{\mathrm{UV}}$ for $\sigma$-type sightlines is higher than for $\zeta$-types, but the standard deviation is relatively large for the poorly determined $\sigma$-type sightlines due to limited $\mathrm{CN}$ data. Exceptionally high $\mathrm{S} / \mathrm{N}$ spectra are needed to probe the $\mathrm{CN}$ content of these most tenuous diffuse clouds.

Our results indicate that the relative abundance of the $\lambda 5780$ DIB carrier increases with respect to the $\lambda 5797$ DIB with an increase in the effective strength of the interstellar UV radiation field. A possible scenario is that a sufficiently large UV flux ionises the molecule whose cation has an electronic transition at $5780 \AA$. The $\lambda 5797$ DIB carrier, on the other hand, is more efficiently destroyed in regions with high $I_{\mathrm{UV}}$ and is only able to survive in the deeper layers of the cloud. The behaviour of the $\lambda \lambda 5780$ and 5797 DIBs is thus strongly dependent on the local effective UV radiation field (and consequently $f_{\mathrm{H}_{2}}$ ) as is to be expected for molecular carriers.

The scatter on the relation between DIB strength and reddening can ultimately be translated to monitor the life cycle of the carriers as they get formed, excited, and destroyed throughout the complex interstellar cloud structure associated with the $\rho$ Oph cloud and the Upp Sco association. The main driver for this cycle is the UV radiation field. More information is needed on the exact conditions of these diffuse clouds to disentangle additional (secondary) drivers. Measurements of additional atomic and molecular lines, such as $\mathrm{C}_{2}, \mathrm{CN}, \mathrm{H}_{2}$, and $\mathrm{CO}$, can improve the constraints on the chemical networks and physical conditions of individual clouds and thus improve our insight in the effects of these parameters on the abundance of DIB carriers. In addition to the two strong narrow DIBs at 5780 and $5797 \AA$ discussed in this work, accurate - high $\mathrm{S} / \mathrm{N}$ - observations of additional narrow and broad diffuse bands are essential to fully probe the effects of changing environmental conditions on the ensemble of carriers giving rise to the DIB spectrum. Moreover, additional detailed studies of the Upp Sco region need to be complemented with similar studies of other specific Galactic and extra-galactic regions to probe a variety and range of different conditions that affect formation and destruction of the DIB carriers.

Acknowledgements. We thank Jos de Bruijne for obtaining the FEROS spectra. D.A.I.V. thanks Paul Groot and Gijs Nelemans for stimulating discussions. P.E. is supported by the NASA Astrobiology Institute. Furthermore, we thank the referee for a very thorough reading of the manuscript and many interesting and helpful suggestions that helped to improve this paper.

\section{References}

Allen, M. M. 1994, ApJ, 424, 754

Black, J. H., \& van Dishoeck, E. F. 1987, ApJ, 322, 412

Bohlin, R. C., Savage, B. D., \& Drake, J. F. 1978, ApJ, 224, 132

Cami, J., Sonnentrucker, P., Ehrenfreund, P., \& Foing, B. H. 1997, A\&A, 326, 822

Cardelli, J. A. 1988, ApJ, 335, 177

Cardelli, J. A., \& Wallerstein, G. 1986, ApJ, 302, 492

Cardelli, J. A., Clayton, G. C., \& Mathis, J. S. 1989, ApJ, 345, 245

Chalabaev, A., \& Maillard, J. P. 1983, A\&A, 127, 279

Chlewicki, G., Van der Zwet, G. P., Van Ijzendoorn, L. J., Greenberg, J. M., \& Alvarez, P. P. 1986, ApJ, 305, 455

Clayton, G. C., \& Cardelli, J. A. 1988, AJ, 96, 695

Cordiner, M. A., Cox, N. L. J., Trundle, C., et al. 2008a, A\&A, 480, L13

Cordiner, M. A., Smith, K. T., Cox, N. L. J., et al. 2008b, A\&A, 492, L5

Corradi, W. J. B., Franco, G. A. P., \& Knude, J. 2004, MNRAS, 347, 1065

Cox, N. L. J., \& Patat, F. 2008, A\&A, 485, L9

Cox, N. L. J., \& Spaans, M. 2006, A\&A, 451, 973

Cox, N. L. J., Kaper, L., Foing, B. H., \& Ehrenfreund, P. 2005, A\&A, 438, 187

Cox, N. L. J., Cordiner, M. A., Cami, J., et al. 2006, A\&A, 447, 991

Cox, N. L. J., Cordiner, M. A., Ehrenfreund, P., et al. 2007, A\&A, 470, 941

Crane, P., Lambert, D. L., \& Sheffer, Y. 1995, ApJS, 99, 107

Crawford, I. A. 1989, MNRAS, 241, 575

de Geus, E. J. 1992, A\&A, 262, 258

de Zeeuw, P. T., Hoogerwerf, R., de Bruijne, J. H. J., Brown, A. G. A., \& Blaauw, A. 1999, AJ, 117, 354

Diplas, A., \& Savage, B. D. 1994, ApJ, 427, 274

Ehrenfreund, P., Cami, J., Jiménez-Vicente, J., et al. 2002, ApJ, 576, L117

Federman, S. R. 1982, ApJ, 257, 125

Federman, S. R., Danks, A. C., \& Lambert, D. L. 1984, ApJ, 287, 219

Federman, S. R., Strom, C. J., Lambert, D. L., et al. 1994, ApJ, 424, 772

Fitzgerald, M. P. 1970, A\&A, 4, 234

Fitzpatrick, E. L., \& Massa, D. 2007, ApJ, 663, 320

Friedman, S. D., York, D. G., McCall, B. J., et al. 2011, ApJ, 727, 33

Galazutdinov, G., Moutou, C., Musaev, F., \& Krełowski, J. 2002, A\&A, 384, 215

Galazutdinov, G. A., Manicò, G., Pirronello, V., \& Krełowski, J. 2004, MNRAS, 355,169

Galazutdinov, G., Lee, B., Song, I., Kazmierczak, M., \& Krełowski, J. 2011, MNRAS, 412, 1259

Grasdalen, G. L., Strom, K. M., \& Strom, S. E. 1973, ApJ, 184, L53

Greene, T. P., \& Young, E. T. 1992, ApJ, 395, 516

Gummersbach, C. A., \& Kaufer, A. 1996, The Hot Star Newsletter, 22, 16

Heckman, T. M., \& Lehnert, M. D. 2000, ApJ, 537, 690

Herbig, G. H. 1993, ApJ, 407, 142

Herbig, G. H. 1995, ARA\&A, 33, 19

Hobbs, L. M., York, D. G., Snow, T. P., et al. 2008, ApJ, 680, 1256

Houk, N. 1982, in Michigan Spectral Survey, Ann Arbor, Dep. Astron., Univ. Michigan, 3, 0

Houk, N., \& Smith-Moore, M. 1988, in Michigan Spectral Survey, Ann Arbor, Dept. of Astronomy, Univ. Michigan, 4, 0

Iglesias-Groth, S., Manchado, A., García-Hernández, D. A., González Hernández, J. I., \& Lambert, D. L. 2008, ApJ, 685, L55

Iglesias-Groth, S., Manchado, A., Rebolo, R., et al. 2010, MNRAS, 407, 2157

Jenniskens, P., Ehrenfreund, P., \& Foing, B. 1994, A\&A, 281, 517

Joseph, C. L., Snow, Jr., T. P., Seab, C. G., \& Crutcher, R. M. 1986, ApJ, 309, 771

Jura, M. 1975, ApJ, 197, 581

Kokkin, D. L., \& Schmidt, W. T. 2006, J. Phys. Chem. A, 110, 6173

Kouwenhoven, M. B. N., Brown, A. G. A., Zinnecker, H., Kaper, L., \& Portegies Zwart, S. F. 2005, A\&A, 430, 137

Krelowski, J. 1989, in Interstellar Dust, ed. L. J. Allamandola, \& A. G. G. M. Tielens, IAU Symp., 135, 67

Krełowski, J., \& Sneden, C. 1995, Diffuse Interstellar Bands in Individual Sightlines, ASSL, 202, 13 
D. A. I. Vos et al.: Diffuse interstellar bands in Upper Scorpius

Krełowski, J, \& Westerlund, B. E. 1988, A\&A, 190, 339

Krełowski, J., Snow, T. P., Seab, C. G., \& Papaj, J. 1992, MNRAS, 258, 693

Krełowski, J., Ehrenfreund, P., Foing, B. H., et al. 1999, A\&A, 347, 235

Larson, K. A., \& Whittet, D. C. B. 2005, ApJ, 623, 897

Lee, D.-H., Pak, S., Van Dyke Dixon, W., \& van Dishoeck, E. F. 2007, ApJ, 655, 940

Lewis, N. K., Cook, T. A., \& Chakrabarti, S. 2005, ApJ, 619, 357

Linnartz, H., Wehres, N., van Winckel, H., et al. 2010, A\&A, 511, L3

Lombardi, M., Lada, C. J., \& Alves, J. 2008, A\&A, 489, 143

Maier, J. P., Walker, G. A. H., Bohlender, D. A., et al. 2011, ApJ, 726, 41

Mamajek, E. E. 2008, Astron. Nachr., 329, 10

Mattila, K. 1986, A\&A, 160, 157

McCall, B. J., Drosback, M. M., Thorburn, J. A., et al. 2010, ApJ, 708, 1628

Megier, A., Aiello, S., Barsella, B., Casu, S., \& Krełowski, J. 2001, MNRAS, 326, 1095

Megier, A., Krełowski, J., \& Weselak, T. 2005, MNRAS, 358, 563

Morton, D. C. 2003, ApJS, 149, 205

Motte, F., Andre, P., \& Neri, R. 1998, A\&A, 336, 150

Pan, K., Federman, S. R., Sheffer, Y., \& Andersson, B. 2005, ApJ, 633, 986

Perryman, M. A. C., Lindegren, L., Kovalevsky, J., et al. 1997, A\&A, 323, L49

Preibisch, T., \& Mamajek, E. 2008, The Nearest OB Association: ScorpiusCentaurus (Sco OB2), ed. B. Reipurth, 235

Preibisch, T., \& Zinnecker, H. 2001, in From Darkness to Light: Origin and Evolution of Young Stellar Clusters, ed. T. Montmerle, \& P. André, ASP Conf. Ser., 243, 791

Press, W., Teukolsky, S., Vetterling, W., \& Flannery, B. 1992, Numerical Recipes in Fortran 77, Second Edition (Cambridge University Press)

Ritchey, A. M., Martinez, M., Pan, K., Federman, S. R., \& Lambert, D. L. 2006, ApJ, 649, 788

Ritchey, A. M., Federman, S. R., \& Lambert, D. L. 2011, ApJ, 728, 36

Ruiterkamp, R., Cox, N. L. J., Spaans, M., et al. 2005, A\&A, 432, 515

Salama, F., Galazutdinov, G. A., Krełowski, J., Allamandola, L. J., \& Musaev, F. A. 1999, ApJ, 526, 265

Salama, F., Galazutdinov, G. A., Krełowski, J., et al. 2011, ApJ, 728, 154

Savage, B. D., Drake, J. F., Budich, W., \& Bohlin, R. C. 1977, ApJ, 216, 291

Schlegel, D. J., Finkbeiner, D. P., \& Davis, M. 1998, ApJ, 500, 525
Schnee, S. L., Ridge, N. A., Goodman, A. A., \& Li, J. G. 2005, ApJ, 634, 442 Seab, C. G., \& Snow, T. P. 1995, ApJ, 443, 698

Sheffer, Y., Rogers, M., Federman, S. R., et al. 2008, ApJ, 687, 1075

Słyk, K., Bondar, A. V., Galazutdinov, G. A., \& Krełowski, J. 2008, MNRAS 390,1733

Sneden, C., Woszczyk, A., \& Krełowski, J. 1991, PASP, 103, 1005

Snow, Jr., T. P., \& Cohen, J. G. 1974, ApJ, 194, 313

Snow, T. P., \& McCall, B. J. 2006, ARA\&A, 44, 367

Snow, T. P., Destree, J. D., \& Welty, D. E. 2008, ApJ, 679, 512

Sollerman, J., Cox, N., Mattila, S., et al. 2005, A\&A, 429, 559

Spaans, M. 1995, Ph.D. Thesis, University of Leiden, Leiden, The Netherlands

Spaans, M. 1996, A\&A, 307, 271

Sujatha, N. V., Shalima, P., Murthy, J., \& Henry, R. C. 2005, ApJ, 633, 257

Thorburn, J. A., Hobbs, L. M., McCall, B. J., et al. 2003, ApJ, 584, 339

Valencic, L. A., Clayton, G. C., \& Gordon, K. D. 2004, ApJ, 616, 912

Van Dishoeck, E. F., \& Black, J. H. 1989, ApJ, 340, 273

van Loon, J. T., Smith, K. T., McDonald, I., et al. 2009, MNRAS, 399, 195

Vollmann, K., \& Eversberg, T. 2006, Astron. Nachr., 327, 862

Walborn, N. R., \& Fitzpatrick, E. L. 1990, PASP, 102, 379

Wegner, W. 2002, Baltic Astron., 11, 1

Welty, D. E., Federman, S. R., Gredel, R., Thorburn, J. A., \& Lambert, D. L. 2006, ApJS, 165, 138

Weselak, T., Galazutdinov, G. A., Musaev, F. A., \& Krełowski, J. 2004, A\&A, 414, 949

Weselak, T., Galazutdinov, G., Musaev, F., \& Krełowski, J. 2008a, A\&A, 479, 149

Weselak, T., Galazutdinov, G. A., Musaev, F. A., \& Krełowski, J. 2008b, A\&A, 484, 381

Whittet, D. C. B. 1974, MNRAS, 168, 371

Wilking, B. A., Gagné, M., \& Allen, L. E. 2008, Star Formation in the $\rho$ Ophiuchi Molecular Cloud, ed. Reipurth, B., 351

Wilking, B. A., Schwartz, R. D., Fanetti, T. M., \& Friel, E. D. 1997, PASP, 109, 549

York, B. A., Ellison, S. L., Lawton, B., et al. 2006, ApJ, 647, L29

Zhou, Z., Sfeir, M. Y., Zhang, L., et al. 2006, ApJ, 638, L105

Zsargó, J., \& Federman, S. R. 2003, ApJ, 589, 319 
Table 1. List of the observed targets.

\begin{tabular}{|c|c|c|c|c|c|c|c|c|c|c|}
\hline Nr. & HD & Hip & $\begin{array}{l}\text { Spectral } \\
\text { type }\end{array}$ & $\begin{array}{c}\text { Right } \\
\text { ascension }\end{array}$ & Declination & $\begin{array}{c}(B-V) \\
(\mathrm{mag})\end{array}$ & $\begin{array}{c}(B-V)_{0} \\
\quad(\mathrm{mag})\end{array}$ & $\begin{array}{l}E_{B-V} \\
(\mathrm{mag})\end{array}$ & $R_{\mathrm{V}}$ & $\begin{array}{c}d^{5} \\
(\mathrm{pc})\end{array}$ \\
\hline 1 & HD 138503 & 76161 & B2/B3IV & $15: 33: 18.91$ & $-25: 01: 37.0$ & -0.048 & -0.22 & 0.17 & & \\
\hline 2 & HD 139094 & 76473 & B8IV & $15: 37: 06.89$ & $-26: 29: 32.6$ & 0.082 & -0.10 & 0.18 & & 289.0 \\
\hline 3 & HD 139160 & 76503 & B9IV & $15: 37: 28.50$ & $-26: 16: 47.5$ & -0.025 & -0.07 & 0.05 & & 184.2 \\
\hline 4 & HD 139486 & 76633 & B9V & $15: 39: 00.06$ & $-19: 43: 57.2$ & 0.033 & -0.07 & 0.10 & & 193.4 \\
\hline 5 & HD 139518 & 76666 & B9.5V & $15: 39: 21.37$ & $-23: 09: 00.8$ & 0.021 & -0.04 & 0.06 & & 93.7 \\
\hline 6 & HD 140543 & 77131 & $\mathrm{~B} 1 \mathrm{Iab} / \mathrm{Ib}$ & $15: 44: 56.66$ & $-21: 48: 53.9$ & -0.013 & -0.19 & 0.18 & $3.16^{w}$ & 735.3 \\
\hline 7 & HD 141180 & 77449 & B9III & $15: 48: 42.90$ & $-27: 34: 56.3$ & 0.002 & -0.08 & 0.08 & & 442.5 \\
\hline 8 & HD 141444 & 77569 & B9.5V & $15: 50: 11.47$ & $-28: 42: 15.6$ & 0.12 & -0.04 & 0.16 & & \\
\hline 9 & HD 141774 & 77677 & B9V & $15: 51: 29.84$ & $-20: 35: 14.5$ & 0.063 & -0.07 & 0.13 & & 202.8 \\
\hline 10 & HD 142096 & 77811 & $\mathrm{~B} 3 \mathrm{~V}$ & $15: 53: 20.06$ & $-20: 10: 01.3$ & -0.032 & -0.20 & 0.17 & & 109.3 \\
\hline 11 & HD 142114 & 77840 & $\mathrm{~B} 2.5 \mathrm{Vn}$ & $15: 53: 36.72$ & $-25: 19: 37.7$ & -0.105 & -0.22 & 0.11 & & 133.0 \\
\hline 12 & HD 142165 & 77858 & B5V & $15: 53: 53.91$ & $-24: 31: 59.4$ & -0.023 & -0.16 & 0.14 & & 127.1 \\
\hline 13 & HD 142184 & 77859 & $\mathrm{~B} 2 \mathrm{~V}$ & $15: 53: 55.86$ & $-23: 58: 41.1$ & -0.052 & -0.24 & 0.19 & & 120.5 \\
\hline 14 & HD 142250 & 77900 & B7V & $15: 54: 30.11$ & $-27: 20: 19.1$ & -0.070 & -0.13 & 0.06 & & 162.9 \\
\hline 15 & HD 142301 & 77909 & B8III/IV & $15: 54: 39.53$ & $-25: 14: 37.5$ & -0.074 & -0.10 & 0.03 & $3.39^{w}$ & 139.7 \\
\hline 16 & HD 142378 & 77939 & B2/B3V & $15: 55: 00.36$ & $-19: 22: 58.5$ & -0.030 & -0.22 & 0.19 & & 191.2 \\
\hline 17 & HD 142669 & 78104 & B2IV-V & $15: 56: 53.08$ & $-29: 12: 50.7$ & -0.192 & -0.24 & 0.05 & & 125.5 \\
\hline 18 & HD 142883 & 78168 & B3V & $15: 57: 40.46$ & $-20: 58: 59.1$ & 0.002 & -0.20 & 0.20 & & 139.7 \\
\hline 19 & HD 142884 & 78183 & B8/B9III & $15: 57: 48.80$ & $-23: 31: 38.3$ & -0.018 & -0.09 & 0.07 & & 122.2 \\
\hline 20 & HD 142983 & 78207 & B8Ia/Iab & $15: 58: 11.37$ & $-14: 16: 45.7$ & -0.089 & -0.02 & -0.07 & & 157.2 \\
\hline 21 & HD 142990 & 78246 & B5V & $15: 58: 34.87$ & $-24: 49: 53.4$ & -0.100 & -0.16 & 0.06 & $3.22^{w}$ & 149.7 \\
\hline 22 & HD 143018 & 78265 & B1V & $15: 58: 51.11$ & $-26: 06: 50.8$ & -0.154 & -0.26 & 0.11 & & 140.8 \\
\hline 23 & HD 143275 & 78401 & B0.3IV & $16: 00: 20.01$ & $-22: 37: 18.2$ & -0.083 & -0.28 & 0.20 & $3.60^{c} 3.09^{w} \mathbf{2 . 8 0} \pm \mathbf{0 . 1 2 ^ { l }}$ & 123.2 \\
\hline 24 & HD 143567 & 78530 & B9V & $16: 01: 55.46$ & $-21: 58: 49.4$ & 0.078 & -0.07 & 0.15 & & 131.9 \\
\hline 25 & HD 143600 & 78549 & B9.5V & $16: 02: 13.56$ & $-22: 41: 15.2$ & 0.085 & -0.04 & 0.12 & & 121.1 \\
\hline 26 & HD 143956 & 78702 & B9V & $16: 04: 00.24$ & $-19: 46: 02.9$ & 0.140 & -0.07 & 0.21 & & 241.5 \\
\hline 27 & HD 144175 & 78809 & B9V & $16: 05: 19.15$ & $-23: 40: 08.8$ & 0.053 & -0.07 & 0.12 & & 131.2 \\
\hline 28 & HD 144217 & 78820 & $\mathrm{~B} 0.5 \mathrm{~V}$ & $16: 05: 26.23$ & $-19: 48: 19.6$ & -0.070 & -0.26 & 0.19 & $4.00^{c} 2.72^{w} \mathbf{3 . 9 0} \pm \mathbf{0 . 2 7 ^ { l }}$ & 162.6 \\
\hline 29 & HD 144218 & 78821 & $\mathrm{~B} 2 \mathrm{~V}$ & $16: 05: 26.55$ & $-19: 48: 06.7$ & -0.007 & -0.24 & 0.23 & & \\
\hline 30 & HD 144334 & 78877 & $\mathrm{~B} 8 \mathrm{~V}$ & $16: 06: 06.38$ & $-23: 36: 22.7$ & -0.077 & -0.11 & 0.03 & & 149.3 \\
\hline 31 & HD 144470 & 78933 & $\mathrm{~B} 1 \mathrm{~V}$ & $16: 06: 48.43$ & $-20: 40: 09.1$ & -0.057 & -0.26 & 0.20 & $3.75^{c} 3.35^{w} 3.37 \pm 0.29^{v} \mathbf{3 . 7 1} \pm \mathbf{0 . 3}^{f} 3.20 \pm 0.13^{l}$ & 129.9 \\
\hline 32 & HD 144569 & 78956 & B9.5V & $16: 07: 04.67$ & $-16: 56: 35.8$ & 0.160 & -0.04 & 0.20 & & 170.9 \\
\hline 33 & HD 144586 & 78968 & B9V & $16: 07: 14.93$ & $-17: 56: 09.7$ & 0.102 & -0.07 & 0.17 & & 159.5 \\
\hline 34 & HD 144661 & 79031 & $\mathrm{~B} 8 \mathrm{IV} / \mathrm{V}$ & $16: 07: 51.89$ & $-24: 27: 44.5$ & -0.052 & -0.10 & 0.05 & & 117.6 \\
\hline 35 & HD 144708 & 79005 & B9V & $16: 07: 36.42$ & $-12: 44: 43.5$ & -0.006 & -0.07 & 0.06 & & 130.5 \\
\hline 36 & HD 144844 & 79098 & B9V & $16: 08: 43.72$ & $-23: 41: 07.5$ & 0.012 & -0.07 & 0.08 & $4.04^{w}$ & 130.7 \\
\hline 37 & HD 144987 & 79199 & B8V & $16: 09: 52.59$ & $-33: 32: 44.9$ & -0.086 & -0.11 & 0.02 & & 132.8 \\
\hline 38 & HD 145353 & 79343 & B9V & $16: 11: 33.52$ & $-27: 09: 03.1$ & 0.012 & -0.07 & 0.17 & & 177.0 \\
\hline 39 & HD 145482 & 79404 & B2V & $16: 12: 18.20$ & $-27: 55: 34.9$ & -0.154 & -0.24 & 0.09 & & 143.5 \\
\hline 40 & HD 145483 & 79399 & B9Vvar & $16: 12: 16.04$ & $-28: 25: 02.3$ & -0.044 & -0.07 & 0.03 & & 91.4 \\
\hline 41 & HD 145502 & 79374 & B2IV & $16: 11: 59.73$ & $-19: 27: 38.6$ & 0.009 & -0.24 & 0.25 & $4.10^{c} 3.48^{w} \mathbf{3 . 5 7} \pm \mathbf{0 . 1 5}^{l}$ & 133.9 \\
\hline 42 & HD 145554 & 79410 & B9V & $16: 12: 21.83$ & $-19: 34: 44.6$ & 0.125 & -0.07 & 0.19 & $3.65 \pm 0.43^{f}$ & 130.2 \\
\hline 43 & HD 145556 & 79437 & B4II/III & $16: 12: 43.56$ & $-28: 19: 18.1$ & 0.015 & -0.15 & 0.16 & & \\
\hline 44 & HD 145631 & 79439 & B9V & $16: 12: 44.10$ & $-19: 30: 10.4$ & 0.133 & -0.07 & 0.20 & $\mathbf{3 . 8 2} \pm \mathbf{0 . 5 3} 3^{l 2}$ & 125.3 \\
\hline 45 & HD 145657 & 79473 & B9.5V & $16: 13: 09.51$ & $-29: 38: 50.1$ & 0.143 & -0.04 & 0.18 & & 370.4 \\
\hline 46 & HD 145792 & 79530 & B6IV & $16: 13: 45.50$ & $-24: 25: 19.5$ & -0.005 & -0.14 & 0.13 & $3.72 \pm 0.75^{v}$ & 138.9 \\
\hline 47 & HD 145964 & 79599 & B9V & $16: 14: 28.88$ & $-21: 06: 27.5$ & 0.001 & -0.07 & 0.07 & & 105.8 \\
\hline 48 & HD 146001 & 79622 & B8V & $16: 14: 53.43$ & $-25: 28: 37.1$ & 0.022 & -0.11 & 0.13 & $3.14^{w}$ & 141.6 \\
\hline 49 & HD 146029 & 79621 & B9V & $16: 14: 53.33$ & $-22: 22: 49.1$ & 0.060 & -0.07 & 0.13 & & 238.1 \\
\hline 50 & HD 146284 & 79740 & B9III/IV & $16: 16: 26.69$ & $-24: 16: 55.0$ & 0.125 & -0.08 & 0.20 & $\mathbf{3 . 1 0}{ }^{f}$ & 263.9 \\
\hline 51 & HD 146285 & 79739 & B8V & $16: 16: 25.17$ & $-24: 59: 19.5$ & 0.201 & -0.11 & 0.31 & $3.83 \pm 0.18^{f}$ & 179.9 \\
\hline 52 & HD 146331 & 79771 & B9V & $16: 16: 50.63$ & $-25: 51: 46.7$ & 0.301 & -0.07 & 0.37 & & 145.8 \\
\hline 53 & HD 146332 & 79775 & B3III & $16: 16: 52.55$ & $-29: 44: 37.4$ & 0.132 & -0.20 & 0.33 & & \\
\hline 54 & HD 146416 & 79785 & B9V & $16: 16: 58.77$ & $-21: 18: 14.9$ & 0.006 & -0.07 & 0.08 & & 125.2 \\
\hline 55 & HD 146706 & 79897 & B9V & $16: 18: 28.26$ & $-23: 16: 27.5$ & 0.103 & -0.07 & 0.17 & $3.36^{w}$ & 170.6 \\
\hline 56 & HD 147009 & 80019 & $\mathrm{RN}$ & $16: 20: 03.98$ & $-20: 02: 41.5$ & 0.256 & -0.01 & 0.27 & & 160.8 \\
\hline 57 & HD 147010 & 80024 & B9II/III & $16: 20: 05.49$ & $-20: 03: 23.0$ & 0.126 & -0.08 & 0.21 & $\mathbf{2 . 8 1} \pm \mathbf{0 . 4 5} 5^{l 2}$ & 143.3 \\
\hline 58 & HD 147103 & 80063 & B9/A0V & $16: 20: 30.26$ & $-20: 07: 03.9$ & 0.338 & -0.04 & 0.38 & & \\
\hline 59 & HD 147165 & 80112 & B1III & $16: 21: 11.32$ & $-25: 35: 34.1$ & 0.097 & -0.26 & 0.36 & $3.80^{c} 3.22^{w} 3.86 \pm 0.52^{v} \mathbf{3 . 6 0} \pm \mathbf{0 . 1 4}^{f} 4.22 \pm 0.20^{l}$ & 225.2 \\
\hline 60 & HD 147196 & 80126 & $\mathrm{~B} 5 / 8 \mathrm{~V}$ & $16: 21: 19.19$ & $-23: 42: 28.7$ & 0.127 & -0.14 & 0.27 & $4.03^{w} \mathbf{3 . 1 0} f$ & 151.3 \\
\hline 61 & HD 147648 & 80338 & B8II & $16: 24: 02.89$ & $-25: 24: 54.0$ & 0.620 & -0.10 & 0.72 & $3.49^{w}$ & 153.8 \\
\hline 62 & HD 147683 & 80405 & B4V & $16: 24: 43.72$ & $-34: 53: 37.5$ & 0.119 & -0.18 & 0.30 & & 591.7 \\
\hline 63 & HD 147701 & 80371 & B5III & $16: 24: 21.32$ & $-25: 01: 31.4$ & 0.500 & -0.16 & 0.66 & $4.05^{c} 3.60^{w} 3.86 \pm 0.24^{v} \mathbf{4 . 0 3} \pm \mathbf{0 . 0 9}^{f}$ & 149.7 \\
\hline 64 & HD 147888 & 80461 & $\mathrm{~B} 3 / \mathrm{B} 4 \mathrm{~V}$ & $16: 25: 24.28$ & $-23: 27: 36.8$ & 0.253 & -0.19 & 0.44 & $4.03^{c} 3.89 \pm 0.20^{v} \mathbf{4 . 0 8} \pm \mathbf{0 . 1 3}^{f}$ & 136.4 \\
\hline
\end{tabular}


D. A. I. Vos et al.: Diffuse interstellar bands in Upper Scorpius

Table 1. continued.

\begin{tabular}{|c|c|c|c|c|c|c|c|c|c|c|}
\hline Nr. & HD & Hip & $\begin{array}{l}\text { Spectral } \\
\text { type }\end{array}$ & $\begin{array}{c}\text { Right } \\
\text { ascension }\end{array}$ & Declination & $\begin{array}{c}(B-V) \\
(\mathrm{mag})\end{array}$ & $\begin{array}{c}(B-V)_{0} \\
(\mathrm{mag})\end{array}$ & $\begin{array}{l}E_{B-V} \\
(\mathrm{mag})\end{array}$ & $R_{\mathrm{V}}$ & $\begin{array}{c}d^{5} \\
(\mathrm{pc})\end{array}$ \\
\hline 65 & HD 147889 & 80462 & B2III/IV & $16: 25: 24.32$ & $-24: 27: 56.6$ & 0.750 & -0.24 & 0.99 & $4.20^{c} 3.68^{w} 3.95 \pm 0.13^{v} \mathbf{4 . 1 9} \pm \mathbf{0 . 0 7 3}$ & 135.9 \\
\hline 66 & HD 147932 & 80474 & B5V & $16: 25: 35.08$ & $-23: 24: 18.8$ & 0.254 & -0.16 & 0.41 & $4.58 \mathbf{4 . 5 8}^{c}$ & 128.9 \\
\hline 67 & HD 147933 & 80473 & BIV & $16: 25: 35.12$ & $-23: 26: 49.8$ & 0.170 & -0.2 & 0.4 & & \\
\hline 68 & HD 147955 & 80493 & B9.5V & $16: 25: 50.66$ & $-26: 34: 06.3$ & 0.206 & -0.07 & 0.28 & & 154.8 \\
\hline 69 & HD 148041 & 80552 & B9IV & $16: 26: 46.59$ & $-33: 04: 23.1$ & 0.316 & -0.07 & 0.39 & & 442.5 \\
\hline 70 & HD 148184 & 80569 & B2Vne & $16: 27: 01.43$ & $-18: 27: 22.5$ & 0.203 & -0.24 & 0.44 & $4.15^{c}$ & 149.9 \\
\hline 71 & HD 148499 & 80778 & B9III & $16: 29: 36.73$ & $-27: 34: 13.7$ & 0.414 & -0.08 & 0.49 & & 346.0 \\
\hline 72 & HD 148579 & 80804 & B9V & $16: 29: 59.20$ & $-25: 08: 52.0$ & 0.245 & -0.07 & 0.31 & $4.01 \pm 0.19^{f}$ & 160.3 \\
\hline 73 & HD 148594 & 80819 & $\mathrm{~B} 8 \mathrm{~V}$ & $16: 30: 15.68$ & $-27: 54: 58.5$ & 0.074 & -0.11 & 0.18 & $\mathbf{3 . 1 0}{ }^{f}$ & 166.9 \\
\hline 74 & HD 148605 & 80815 & B3V & $16: 30: 12.48$ & $-25: 06: 54.8$ & -0.132 & -0.20 & 0.07 & $3.18 \pm 0.52^{w}$ & 120.5 \\
\hline 75 & HD 148860 & 80940 & B9.5III & $16: 31: 38.71$ & $-17: 42: 48.3$ & 0.132 & -0.05 & 0.18 & & 207.0 \\
\hline 76 & HD 149367 & 81211 & B8/9IV/V & $16: 35: 15.21$ & $-26: 28: 42.1$ & 0.105 & -0.07 & 0.18 & $3.49 \pm 0.10^{w}$ & \\
\hline 77 & HD 149387 & 81236 & B7II/III & $16: 35: 29.22$ & $-30: 16: 03.5$ & 0.141 & -0.12 & 0.26 & & \\
\hline 78 & HD 149438 & 81266 & B0V & $16: 35: 52.95$ & $-28: 12: 57.7$ & -0.198 & -0.30 & 0.10 & & 131.8 \\
\hline 79 & HD 149757 & 81377 & O9.5Vn & $16: 37: 09.54$ & $-10: 34: 01.5$ & 0.020 & -0.30 & 0.32 & $3.09^{c} 2.55 \pm 0.24^{v} \mathbf{3 . 0 8} \pm \mathbf{0 . 1 9}{ }^{f}$ & 140.4 \\
\hline 80 & HD 149883 & 81487 & B9V & $16: 38: 36.29$ & $-26: 59: 21.6$ & 0.128 & -0.07 & 0.20 & & 237.5 \\
\hline 81 & HD 149914 & 81474 & B9.5IV & $16: 38: 28.65$ & $-18: 13: 13.7$ & 0.231 & -0.04 & 0.27 & & 165.0 \\
\hline 82 & HD 150514 & 81785 & B8III & $16: 42: 17.41$ & $-20: 11: 13.3$ & 0.103 & -0.10 & 0.20 & & \\
\hline 83 & HD 150814 & 81941 & B9.5V & $16: 44: 17.66$ & $-22: 31: 24.9$ & 0.129 & -0.04 & 0.17 & & 183.2 \\
\hline 84 & HD 151012 & 82069 & B9.5V & $16: 45: 48.46$ & $-26: 38: 57.9$ & 0.044 & -0.04 & 0.08 & & 110.1 \\
\hline 85 & HD 151346 & 82217 & B8II & $16: 47: 46.53$ & $-23: 58: 27.5$ & 0.386 & -0.10 & 0.49 & $3.70^{w} \mathbf{3 . 8 1} \pm \mathbf{0 . 1 0}{ }^{f}$ & 253.8 \\
\hline 86 & HD 151496 & 82271 & B9V & $16: 48: 36.25$ & $-21: 56: 28.1$ & 0.191 & -0.07 & 0.26 & & 298.5 \\
\hline 87 & HD 152516 & 82734 & B2III & $16: 54: 38.40$ & $-21: 52: 46.6$ & 0.061 & -0.24 & 0.30 & & \\
\hline 88 & HD 152655 & 82822 & B9III & $16: 55: 32.54$ & $-21: 34: 10.2$ & 0.100 & -0.08 & 0.18 & & 425.5 \\
\hline 89 & HD 152657 & 82839 & B8II & $16: 55: 44.38$ & $-25: 32: 02.1$ & 0.004 & -0.10 & 0.10 & & 361.0 \\
\hline
\end{tabular}

Notes. Columns 1 to 8 give the Henry-Draper (HD) number, Hipparcos (Hip) number, spectral type, right ascension \& declination, colour $(B-V)$, intrinsic stellar colour $(B-V)_{0}$, and reddening $E_{(B-V)}$. Spectral types are from the Michigan Spectral Catalog of HD stars (Houk 1982; Houk \& Smith-Moore 1988). Intrinsic colours, $(B-V)_{0}$, are taken from Fitzgerald 1970. $B$ and $V$ photometric magnitudes are taken from Tycho-2 catalog and converted to the Johnson colour index $B-V$ (using Bessell 2000 and Mamajek et al. 2002); subsequently $E_{B-V}=(B-V)-(B-V)_{0}$. Typical error on $E_{(B-V)}$ is $\sim 0.03 \mathrm{mag}$ (see Sect. 3). Col. 9 lists the total-to-selective visual extinction ratio $\mathrm{R}_{v}$ from Fitzpatrick \& Massa (2007) [f], Valencic et al. (2004) [v], Wegner (2002) [w], Clayton \& Cardelli (1988); Cardelli et al. (1989) [c], Lewis et al. (2005) [1], and Larson \& Whittet (2005) [12] Col. 10 gives the parallax distance $d$ (pc) from Hipparcos (Perryman et al. 1997). 


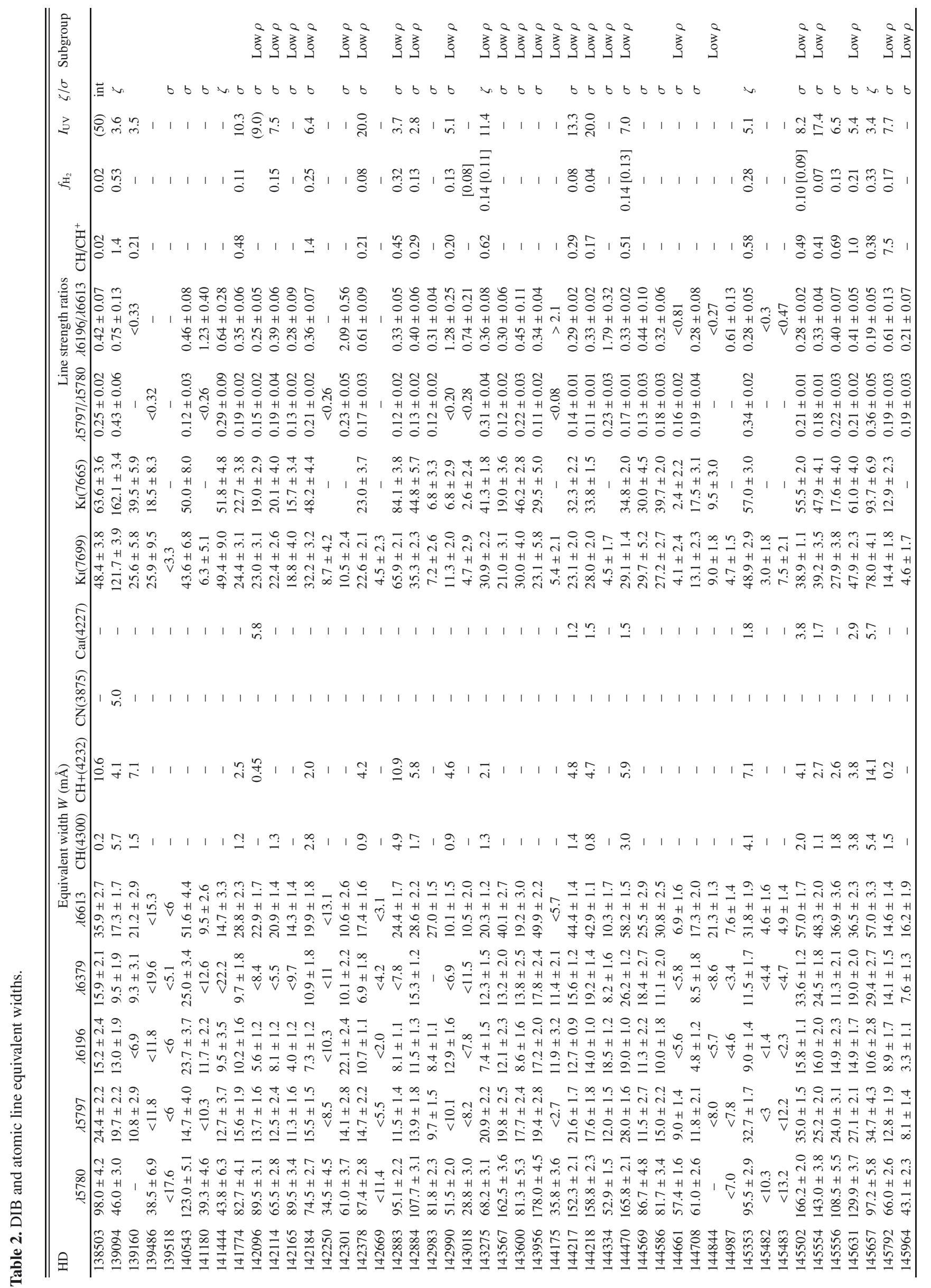


D. A. I. Vos et al.: Diffuse interstellar bands in Upper Scorpius

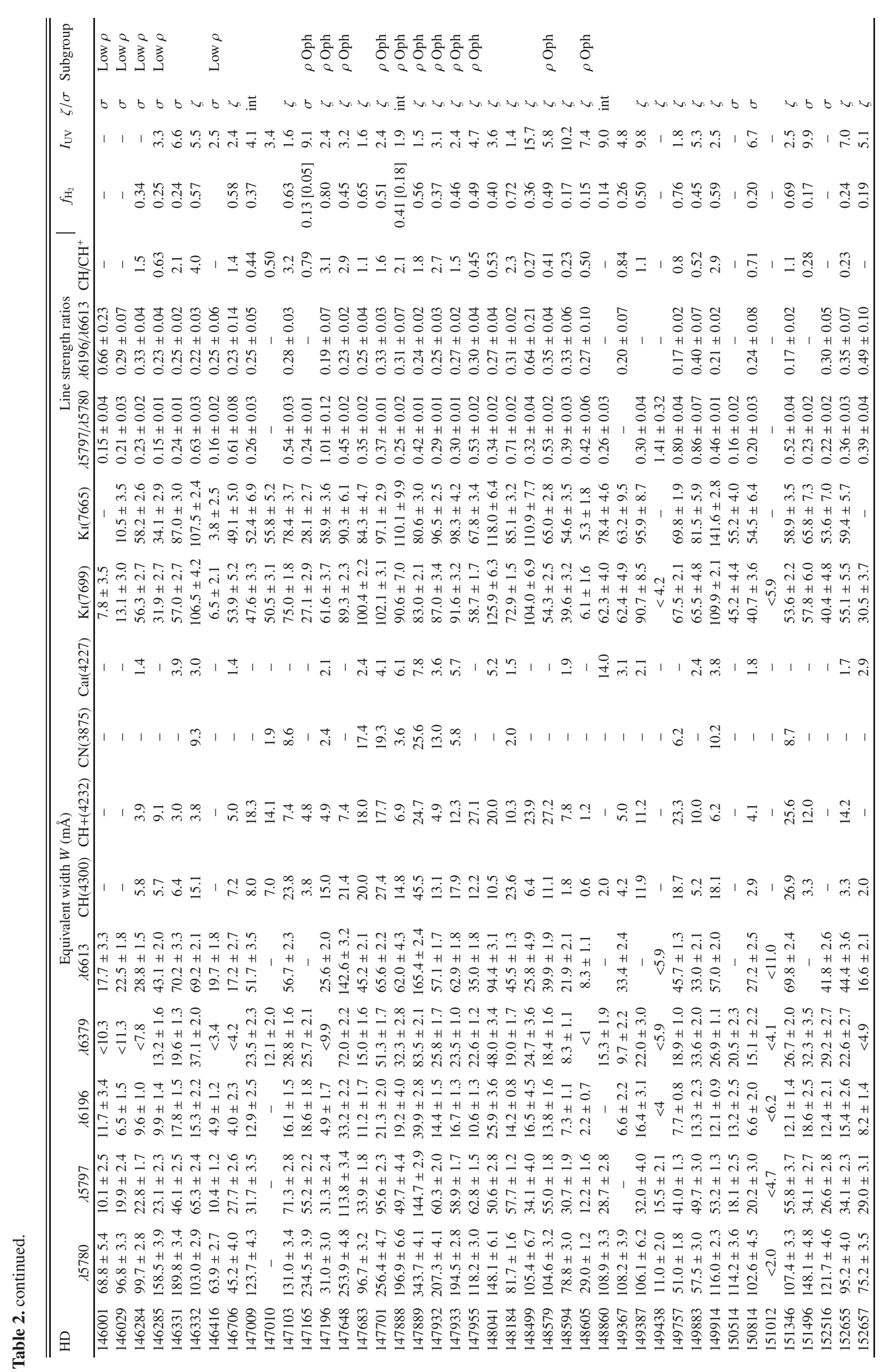

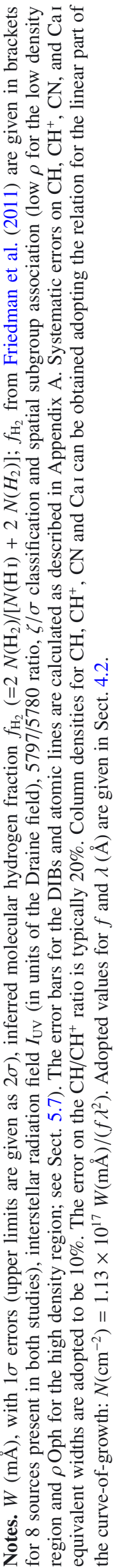


A\&A 533, A129 (2011)

Table 5. Central radial velocities (in the heliocentric frame) for selected atomic and molecular lines.

\begin{tabular}{|c|c|c|c|c|c|c|c|c|}
\hline \multirow[b]{2}{*}{ HD } & \multicolumn{8}{|c|}{ Radial velocities $\left(\mathrm{km} \mathrm{s}^{-1}\right)$} \\
\hline & $v_{\mathrm{CH}^{+}}$ & $v_{\mathrm{CH}}$ & $v_{\mathrm{CN}}$ & $v_{\mathrm{CaI}}$ & $v_{\mathrm{KI}}$ & & $v_{\mathrm{NaI}}$ & \\
\hline 138503 & -6 & -6 & & & -6 & -6 & -6 & \\
\hline 139094 & -8 & -9 & -11 & -9 & -9 & -8 & -10 & \\
\hline 139160 & -7 & -7 & & & -8 & -8 & -9 & \\
\hline 139486 & & & & & -13 & -13 & -13 & \\
\hline 139518 & & & & & & & -15 & \\
\hline 140543 & -10 & & & & -13 & -13 & -14 & \\
\hline 141180 & & & & & & & -7 & $-17,+11$ \\
\hline 141444 & & & & & -4 & -4 & -5 & \\
\hline 141774 & -10 & -9 & & & -10 & -10 & -9 & $-29,-18,+16$ \\
\hline 142096 & & & & & -11 & -10 & -11 & \\
\hline 142114 & & -8 & & & -9 & -8 & -9 & -14 \\
\hline 142165 & & & & & -10 & -9 & -10 & \\
\hline 142184 & & -12 & & & -11 & -12 & -14 & -33 \\
\hline 142250 & & & & & & & -12 & \\
\hline 142301 & & & & & & & -15 & \\
\hline 142378 & -14 & & & & -13 & -13 & -12 & \\
\hline 142669 & & & & & & & -9 & -21 \\
\hline 142883 & -15 & -14 & & & -14 & -13 & -13 & \\
\hline 142884 & -19 & -18 & & & -14 & -13 & -15 & \\
\hline 142983 & & & & & -12 & -14 & -12 & -24 \\
\hline 142990 & -17 & -15 & & & -13 & -14 & -15 & \\
\hline 143018 & & & & & & & -15 & \\
\hline 143275 & -13 & & & & -12 & -11 & -12 & \\
\hline 143567 & & & & & -10 & -10 & -10 & -22 \\
\hline 143600 & & & & & -12 & -13 & -12 & -23 \\
\hline 143956 & & & & & -8 & -8 & -10 & -22 \\
\hline 144175 & & & & & & & -11 & -21 \\
\hline 144217 & -11 & -10 & & -10 & -10 & -10 & -10 & -23 \\
\hline 144218 & -11 & & & -10 & -10 & -9 & -10 & -23 \\
\hline 144334 & & & & & & & -9 & -22 \\
\hline 144470 & -11 & -10 & & & -10 & -10 & -9 & -23 \\
\hline 144569 & -12 & & & & -12 & -11 & -12 & -27 \\
\hline 144586 & -11 & -9 & & & -10 & -10 & -10 & -26 \\
\hline 144661 & & & & & & & -9 & -22 \\
\hline 144708 & & & & & -13 & -13 & -12 & \\
\hline 144844 & & & & & -10 & -10 & -9 & -23 \\
\hline 144987 & & & & & & -10 & -10 & -22 \\
\hline 145353 & -2 & -2 & & -7 & -4 & -5 & -5 & -21 \\
\hline 145482 & & & & & & -11 & -1 & -20 \\
\hline 145483 & & & & & & -11 & & -25 \\
\hline 145502 & -11 & -9 & & -10 & -10 & -10 & -10 & -25 \\
\hline 145554 & -11 & & & -13 & -10 & -10 & -10 & -25 \\
\hline 145556 & -3 & -4 & & & -4 & -4 & -4 & -25 \\
\hline
\end{tabular}


D. A. I. Vos et al.: Diffuse interstellar bands in Upper Scorpius

Table 5. continued.

\begin{tabular}{|c|c|c|c|c|c|c|c|c|}
\hline \multirow[b]{2}{*}{ HD } & \multicolumn{8}{|c|}{ Radial heliocentric velocities $\left(\mathrm{km} \mathrm{s}^{-1}\right)$} \\
\hline & $v_{\mathrm{CH}^{+}}$ & $v_{\mathrm{CH}}$ & $v_{\mathrm{CN}}$ & $v_{\mathrm{CaI}}$ & $v_{\mathrm{KI}}$ & & $v_{\mathrm{NaI}}$ & \\
\hline 145631 & -8 & -8 & & -9 & -9 & -9 & -9 & -25 \\
\hline 145657 & -6 & -4 & & -3 & -3 & -4 & -5 & -19 \\
\hline 145792 & -5 & & & & -7 & -7 & -7 & -22 \\
\hline 145964 & & & & & -9 & -10 & -9 & -22 \\
\hline 146001 & -5 & & & & $-8 ?$ & $-9 ?$ & -6 & -21 \\
\hline 146029 & & & & & -9 & -9 & -8 & -23 \\
\hline 146284 & -8 & -8 & & -8 & -9 & -8 & -8 & -24 \\
\hline 146285 & -6 & -5 & & & -5 & -6 & -6 & -22 \\
\hline 146331 & -7 & -7 & & -9 & -8 & -8 & -9 & \\
\hline 146332 & -1 & -3 & -4 & -4 & -4 & -3 & -5 & +14 \\
\hline 146416 & & & & & -11 & -10 & -9 & -25 \\
\hline 146706 & -8 & -7 & & -7 & -8 & -8 & -8 & \\
\hline 147009 & -9 & -9 & & & -9 & -9 & -9 & \\
\hline 147010 & -10 & -10 & & & -11 & -9 & -9 & \\
\hline 147103 & -8 & -8 & -8 & & -9 & -8 & -9 & -24 \\
\hline 147165 & -7 & -7 & & & -7 & -7 & -6 & \\
\hline 147196 & -7 & -7 & -7 & & -7 & -8 & -9 & \\
\hline 147648 & -7 & -7 & & & -7 & -7 & -7 & \\
\hline 147683 & -1 & 0 & -1 & -1 & -1 & -1 & +1 & -18 \\
\hline 147701 & -7 & -6 & -6 & -6 & -7 & -7 & & \\
\hline 147888 & -9 & -8 & -9 & -9 & -9 & -8 & -9 & \\
\hline 147889 & -8 & -7 & -7 & -8 & -8 & -8 & -9 & \\
\hline 147932 & -7 & -7 & -8 & -8 & -9 & -8 & -9 & \\
\hline 147933 & -8 & -8 & -9 & -9 & -9 & -8 & -9 & \\
\hline 147955 & -6 & -5 & & & -7 & -6 & -6 & \\
\hline 148041 & -1 & -1 & & -4 & -1 & 0 & -1 & \\
\hline 148184 & -11 & -11 & -11 & -12 & -11 & -12 & -12 & \\
\hline 148499 & -4 & -4 & & & -4 & -4 & -4 & +12 \\
\hline 148579 & -5 & -5 & & & -6 & -5 & -6 & \\
\hline 148594 & -5 & -4 & & -6 & -5 & -5 & -5 & \\
\hline 148605 & -8 & & & -8 & -9 & & -8 & \\
\hline 148860 & & -12 & & -7 & -12 & -12 & -10 & -55 \\
\hline 149367 & -4 & -4 & & & -4 & -4 & -5 & \\
\hline 149387 & -3 & -3 & & -2 & -4 & -3 & -4 & \\
\hline 149438 & & & & & & $-8 ? ?$ & -8 & \\
\hline 149757 & -15 & -15 & -15 & & -15 & -15 & -15 & -27 \\
\hline 149883 & -5 & -4 & & -6 & -4 & -4 & -5 & \\
\hline 149914 & -12 & -11 & -12 & -10 & -11 & -11 & -10 & \\
\hline 150514 & -8 & & & & -7 & -7 & -9 & $-27,+7$ \\
\hline 150814 & -12 & & & & -14 & -14 & -13 & +2 \\
\hline 151012 & & $-20 ?$ & & & $-19 ?$ & $-22 ?$ & & \\
\hline 151346 & -7 & -7 & -7 & & -7 & -7 & -8 & \\
\hline 151496 & -9 & -8 & & & -9 & -9 & -10 & \\
\hline 152516 & -7 & & & & -6 & -6 & -7 & \\
\hline 152655 & -10 & -9 & & -8 & -10 & -10 & -9 & \\
\hline 152657 & & & & & & & -4 & \\
\hline
\end{tabular}

Notes. With an instrumental profile $F W H M$ of about $6-7 \mathrm{~km} \mathrm{~s}^{-1}$ and pixels of $2 \mathrm{~km} \mathrm{~s}^{-1}$, the accuracy of the measured radial velocity is $1-2 \mathrm{~km} \mathrm{~s}{ }^{-1}$. 


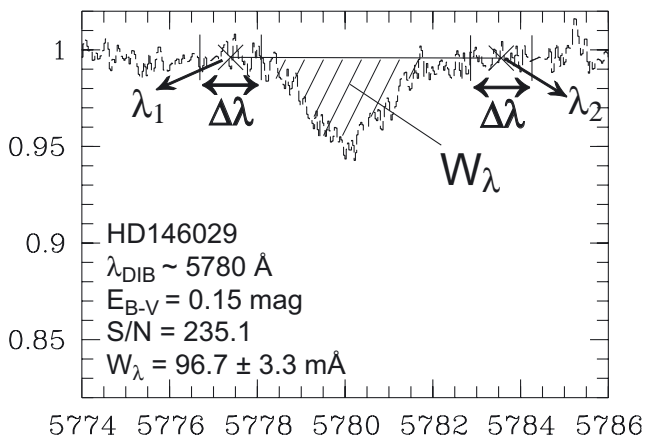

Fig. A.1. Typical example illustrating the determination of the DIB equivalent width.

\section{Appendix A: Error on equivalent width}

For the equivalent width measurement $\left(W=\int_{\lambda_{1}}^{\lambda_{2}}\left(\frac{F_{\mathrm{c}}-F_{\lambda}}{F_{\mathrm{c}}}\right)\right)$, two integration borders $\lambda_{1}$ and $\lambda_{2}$ are selected for which the average intensity in the continuum is derived in a window of width $\Delta \lambda_{\mathrm{c}}=1.4 \AA$ centred on the borders (Fig. A.1).

The broader DIBs at 5778 and $5796 \AA$ underlying the strong 5780 and $5797 \AA$ DIBs are largely removed from the spectra in the local continuum normalisation. They are very weak, even for high $E_{(B-V)}$, and do not significantly contaminate the $W$ measurements of the 5780 and $5797 \AA$ DIBs.

Taking into account both the noise of the measured flux ( $\left.\sigma_{\text {noise }}\right)$ and the uncertainty in estimating the continuum $\left(\sigma_{\mathrm{c}}\right)$, the error on the equivalent width is given according to Chalabaev \& Maillard (1983) [CM83], in our notation:

$$
\begin{aligned}
\sigma_{W}^{2}= & \sigma_{\text {noise }}^{2}+\sigma_{\text {continuum }}^{2} \\
& \text { via Eq. (A.9) from CM83: } \\
= & M \frac{\delta \lambda^{2}}{S N R}\left(\frac{F_{j}}{F_{\mathrm{c}}}\right)^{2}+\frac{\sigma\left(F_{\mathrm{c}}\right)^{2}}{F_{\mathrm{c}}}(\Delta \lambda-W)^{2} \\
= & \Delta \lambda_{\text {line }} \frac{\delta \lambda}{S N R^{2}}\left(F_{j}\right)^{2}+\frac{\delta \lambda}{\Delta \lambda_{\mathrm{c}}} \frac{1}{S N R^{2}}\left(\Delta \lambda_{\text {line }}-W\right)^{2} \\
= & \frac{\delta \lambda}{S N R^{2}}\left(\Delta \lambda_{\text {line }} F_{j}^{2}+\frac{\left(\Delta \lambda_{\text {line }}-W\right)^{2}}{\Delta \lambda_{\mathrm{c}}}\right) \\
= & \frac{\Delta \lambda \delta \lambda}{S N R^{2}}\left(F_{j}+\frac{(\Delta \lambda-W)}{\Delta \lambda^{2}}\right) \\
\approx & \frac{\Delta \lambda \delta \lambda}{S N R^{2}}\left(1+\frac{(\Delta \lambda-W)}{\Delta \lambda^{2}}\right) \\
\sigma_{W} \approx & \frac{\sqrt{\Delta \lambda \delta \lambda}}{S N R} \sqrt{2}
\end{aligned}
$$

where we used the integration range $\lambda_{2}-\lambda_{1}=M \delta \lambda=\Delta \lambda_{\text {line }}$, $S N R$ is the signal-to-noise ratio (per pixel), $\frac{\sigma_{F_{\mathrm{c}}}^{2}}{F_{\mathrm{c}}^{2}}=\frac{1}{N_{\mathrm{c}} S N R^{2}}, N_{\mathrm{c}}=$ $\Delta \lambda / \delta \lambda, \delta \lambda$ is the spectral dispersion in $\AA /$ pix, $M$ the number of pixels in the integration range, and we take $\Delta \lambda_{\mathrm{c}} \approx \Delta \lambda_{\text {line }}=\Delta \lambda$. In Eq. (A.6) the last term is approximately 2 as for shallow-weak lines $W \ll \Delta \lambda=3 \AA$. We have $\delta \lambda=0.03 \AA, F W H M \approx 1 \AA$ (typical for a narrow DIB), so that $\Delta \lambda \approx 3 F W H M$. This error estimate yields similar results as when applying the error estimate in the weak line limit by Vollmann \& Eversberg (2006) (Eq. (A.X)): $\sigma_{W}=\sqrt{2} \frac{(\Delta \lambda-W)}{S N R}=\frac{\sqrt{2}}{S N R} \sqrt{\Delta \lambda \delta \lambda}$.

For the strong narrow $\mathrm{K}_{\mathrm{I}}$ line the approximation in Eq. (A.6) is not valid and the given $\sigma_{W}$ will be an upper limit to the true $\sigma_{W}$.
Table B.1. Overview of possible significant contamination from stellar lines to the interstellar line equivalent width measurements.

\begin{tabular}{lccc}
\hline \hline Measured line & Contaminating line & Spectral types & Contamination $W$ \\
\hline $5780 \AA$ & Fe II $(5780.1 \AA)$ & B5V-A0V & $13 \mathrm{~m} \AA$ \\
& " & $<$ B5V & $<4 \mathrm{~m} \AA$ \\
& He I $(5780.5 \AA)$ & O9V & $\sim 2 \mathrm{~m} \AA$ \\
$6379 \AA$ & Ne II $(6379.6 \AA)$ & B0V, B1 V, B2V & $8,22,8 \mathrm{~m} \AA$ \\
$\mathrm{CH}(4300.3 \AA)$ & Ti II $(4300.0 \AA)$ & B5V & $4 \mathrm{~m} \AA$ \\
& $\prime \prime$ & $\mathrm{A} 0 \mathrm{~V}$ & $161 \mathrm{~m} \AA$ \\
$\mathrm{CH}^{+}(4232.5 \AA)$ & $\mathrm{Fe}$ II $(4232.8 \AA)$ & $\mathrm{B} 2 \mathrm{~V}$ & $<12 \mathrm{~m} \AA$ \\
& $\prime \prime$ & $\mathrm{B} 5 \mathrm{~V}$ & $\sim 98 \mathrm{~m} \AA$ \\
& & $\mathrm{A} 0 \mathrm{~V}$ & $\sim 300 \mathrm{~m} \AA$ \\
& $\mathrm{Si}$ II $(4233.2 \AA)$ & $\mathrm{B} 2 \mathrm{~V}$ & $<12 \mathrm{~m} \AA$ \\
\hline
\end{tabular}

\section{Appendix B: Contamination with the photospheric spectrum}

In order to evaluate possible contamination from stellar lines to the measured equivalent widths of the interstellar lines (both DIBs and molecules) we have checked synthetic spectra of $\mathrm{B}$ main-sequence (A0V, B5V, B2V, B0V, O9V) stars for the presence of atmospheric lines in the regions of interest in this study (see Gummersbach \& Kaufer 1996, for details on the models). A brief overview of the expected contamination is given in Table B.1.

For the 5797, $6196 \AA$ DIBs no contamination above the $2 \mathrm{~m} \AA$ level is expected for these B-type main-sequence stars. The N II line at $6610.6 \AA$ present in spectra of O9V to B2V stars is clearly separated from the $6613 \AA \mathrm{DIB}$ at the spectral resolving power of FEROS. Both the O II line at $4303.6 \AA$ and the Fe II line at $4303.2 \AA$ are also well resolved from the $\mathrm{CH} 4300.3 \AA$ line (the rest wavelength velocity difference is more than $\left.200 \mathrm{~km} \mathrm{~s}^{-1}\right)$. For $\mathrm{CH}(4300.3 \AA)$ the contamination in A0V spectra may be severe due to the presence of Ti II, however in that case also the Fe II line at 4303.2 $\AA$ should be clearly present (with $W \sim 200 \mathrm{~m} \AA$ ). Strong Fe I lines appear in A0V spectra at $3872.5 \AA$ and $3878.6 \AA$, however, these are also clearly separated from the interstellar CN line (3874.6 $\AA$ ). The velocity profile plots cover a region of $\pm 0.6 \AA$ around the $\mathrm{CN}$ line. Also the weak to strong ( $W_{\text {total }} \sim 34$ to $\sim 185 \mathrm{m \AA}$ ) $\mathrm{C}_{\text {II }}$ lines around 3876.0-3876.7 $\AA$ fall outside the plotted window. Only the Ne II line at $3875.3 \AA$ is relatively close to the $\mathrm{CN}$ line (with rest wavelength difference of $54 \mathrm{~km} \mathrm{~s}^{-1}$ ).

There are several ways to investigate the potential problem of contamination. For example, the line widths of all stellar lines are set by the rotational velocity $(v \sin i)$, whereas the di-atomic lines have intrinsically small widths, and can thus be distinguished from each other. Also, the radial velocities of the stellar and interstellar lines are (in virtually all cases) different. Therefore, any (di)atomic line with a velocity significantly different from e.g. that of the $\mathrm{K}_{\mathrm{I}}$ doublet (no contamination expected) is suspect. Furthermore, in the case of relatively strong $\mathrm{CH}^{+}$line, the $\mathrm{CH}^{+}$transition at $3957.7 \AA$ can be used to verify and confirm the abundance of $\mathrm{CH}^{+}$. For the relatively broad DIBs this differentiation due to velocity differences is less secure. Fortunately, the contamination expected at the DIB wavelengths is very low. 


\section{Appendix C: Correlation plots}
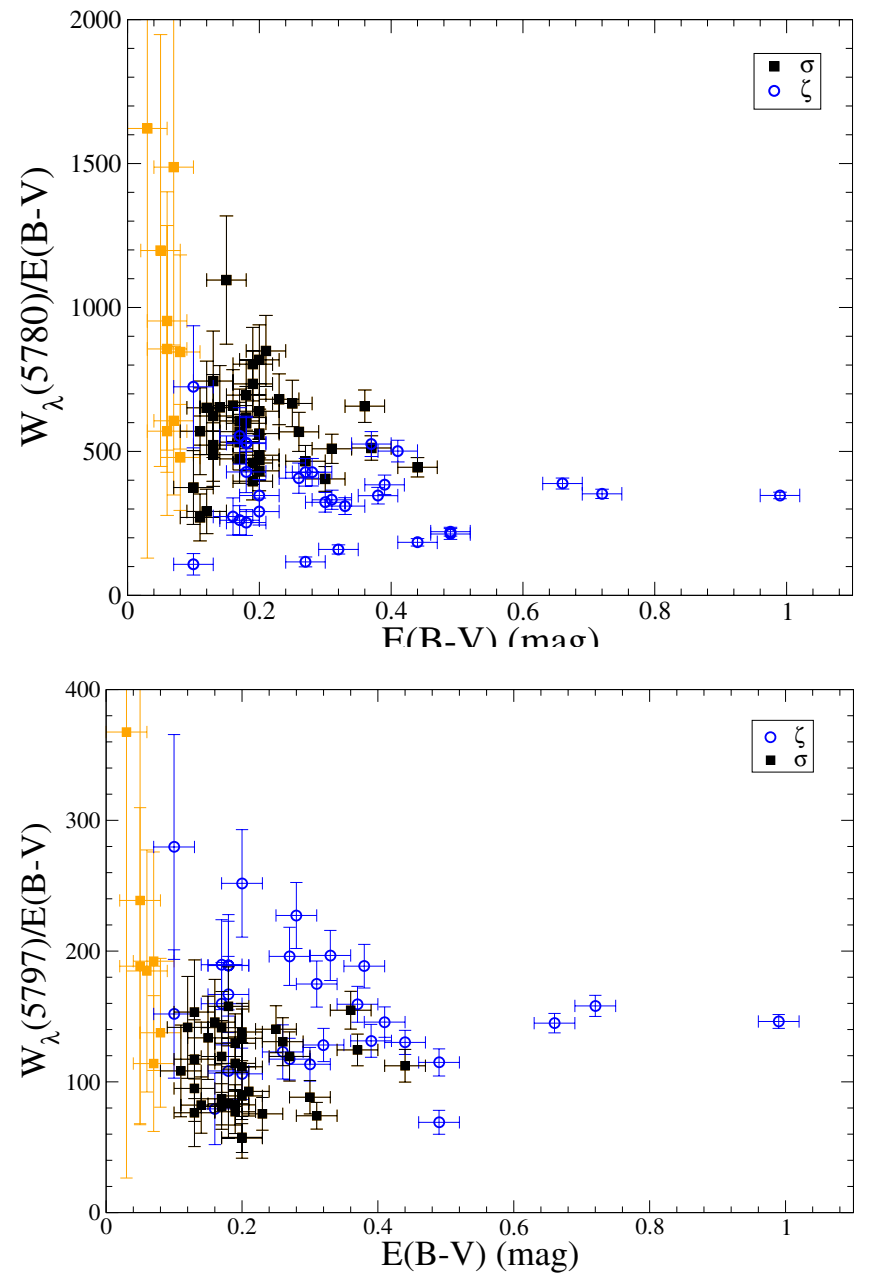

Fig. C.1. Equivalent width per unit reddening versus $E_{(B-V)}$ for the 5780 and $5797 \AA$ DIBs. Reduced chi-squared for linear fits are indicated in the respective panels. This plot reveals an intrinsic scatter on the relationship between the DIB strength and amount of dust in Upper Scorpius. Sightlines with $E_{(B-V)}<0.1 \mathrm{mag}$ (orange/grey squares) are omitted for calculating the correlation coefficient; $r_{5780}=-0.32$ and $r_{5797}=0.07$.
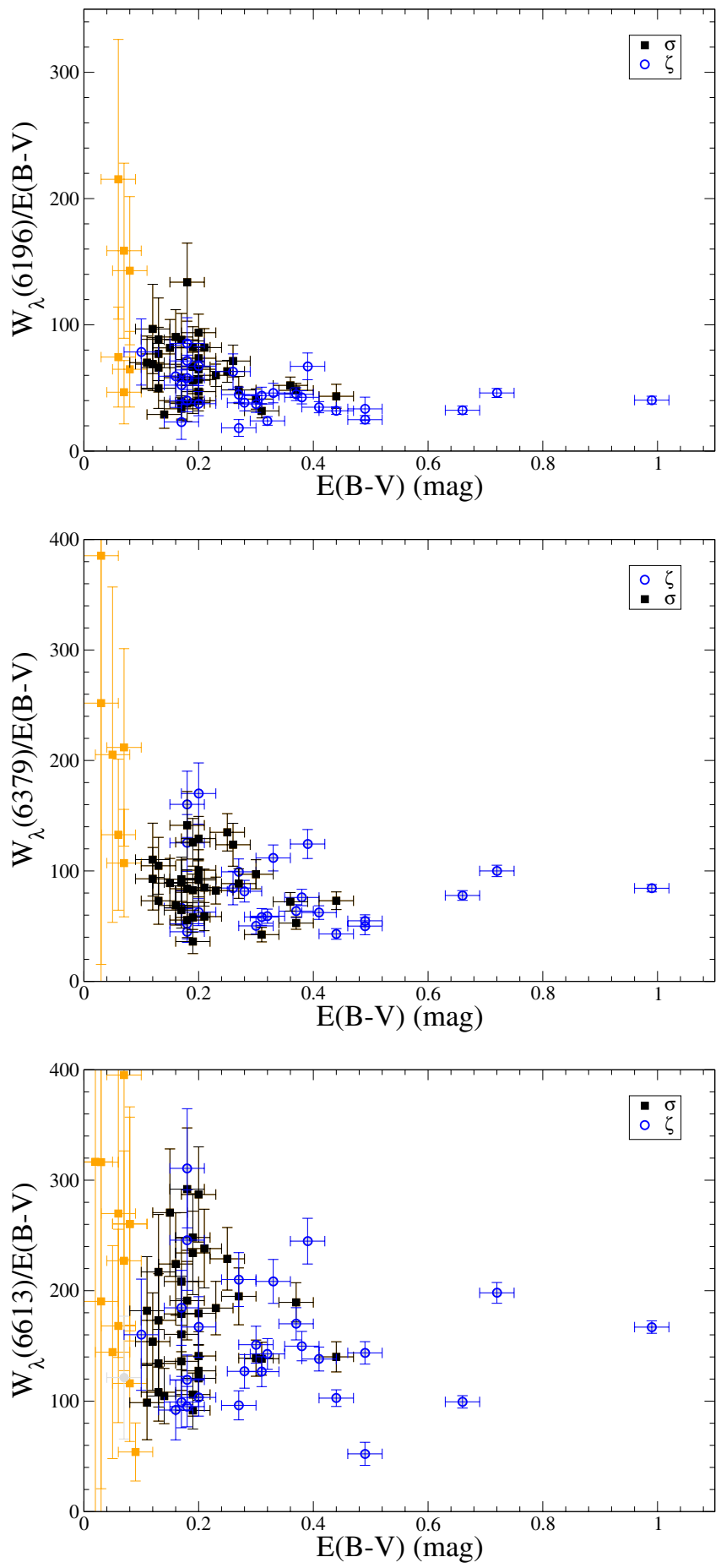

Fig. C.2. Equivalent widths per unit reddening versus $E_{(B-V)}$ for the 6196, 6379, and 6613 A DIBs. Reduced chi-square for linear fits are indicated in the respective panels. This plot reveals an intrinsic scatter on the relationship between the DIB strength and amount of dust in Upper Scorpius. Sightlines with $E_{(B-V)}<0.1 \mathrm{mag}$ (orange/grey squares) are omitted for calculating the correlation coefficient; $r_{6196}=-0.41$, $r_{6379}=-0.17$, and $r_{6613}=-0.12$. 

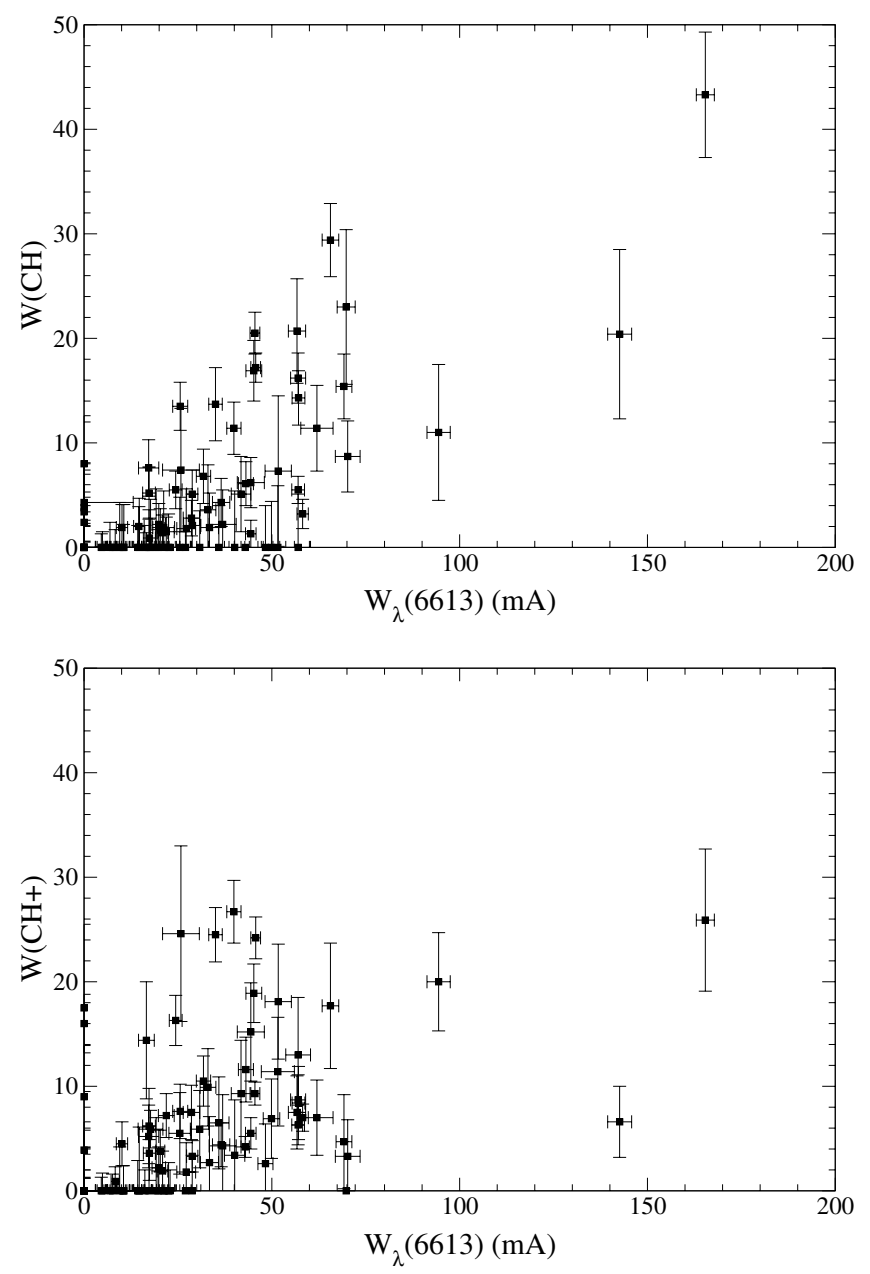

Fig. C.3. Equivalent widths for $\mathrm{CH}($ top $)$ and $\mathrm{CH}^{+}$(bottom) are plotted versus $W(6613)$, the strength of the $6613 \AA$ DIB. See Sect. 5.4.

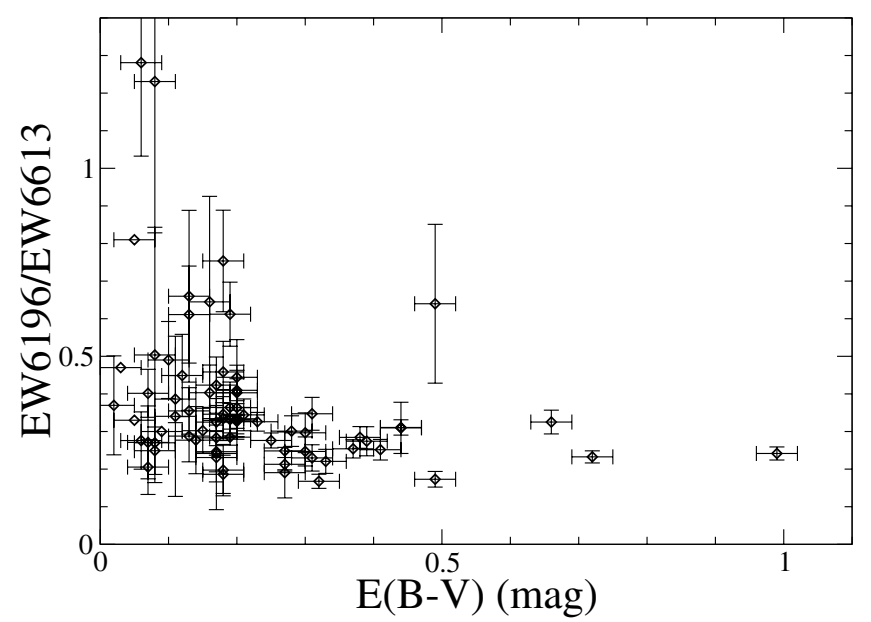

Fig. C.4. The $W(6196) / W(6613)$ ratio plotted against $E_{(B-V)}$. Contrary to the $(5797) / W(5780)$ ratio discussed in Sect. 5.7 this ratio is less sensitive to reddening. 

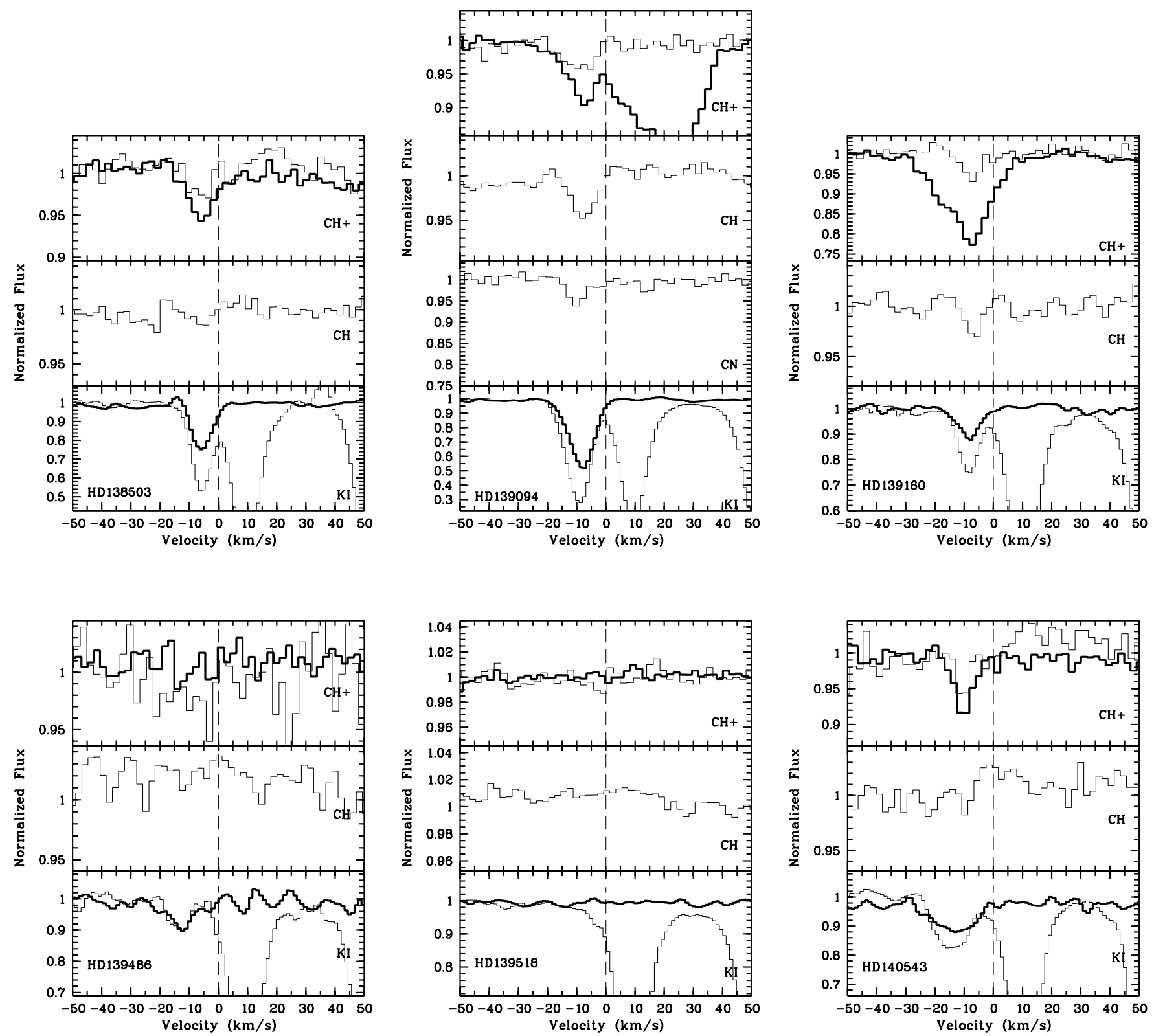

Fig. D.1. Observed profiles for the interstellar lines of $\mathrm{CH}^{+}, \mathrm{CH}$, and $\mathrm{K}_{\mathrm{I}}$ in sightlines probing the Upp Sco region. HD numbers correspond to those given in Table 1. In the bottom panel the weaker $\mathrm{K}_{\mathrm{I}}$ doublet line at $7699 \AA$ is over-plotted (thick solid line) on top of the stronger (telluric contaminated) $\mathrm{K}_{\mathrm{I}}$ doublet line at $7665 \AA$. The $\mathrm{Ca}$ I and $\mathrm{CN}$ lines are included only if potentially detected. The $\mathrm{CH}$ and $\mathrm{CH}^{+}$lines are shown for all lines-of-sight. The stronger $\mathrm{CH}^{+}$line at $4232 \AA$ is over-plotted (thick solid line) on top of the $3957 \AA$ line. 

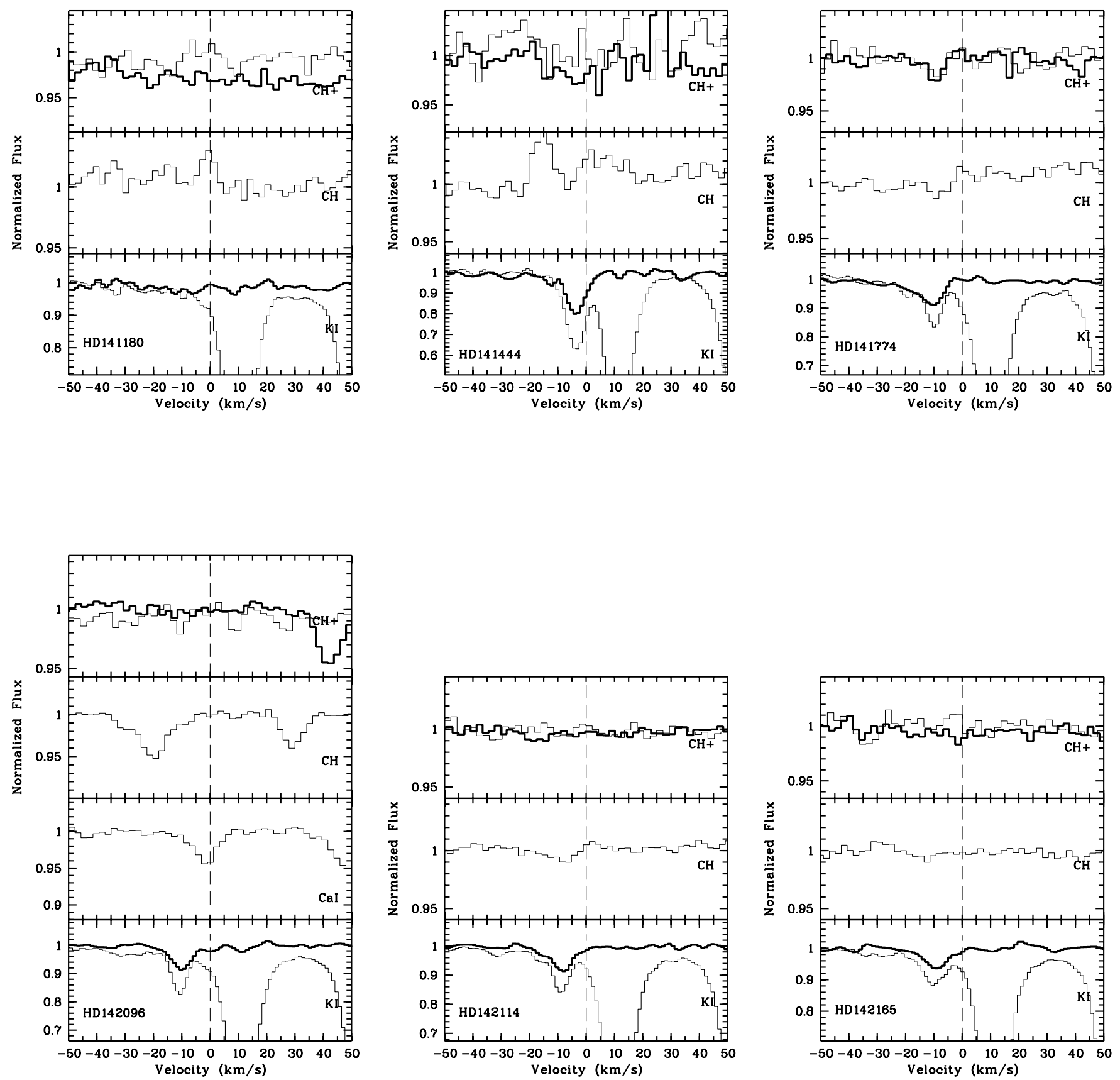

Fig. D.1. continued. 
D. A. I. Vos et al.: Diffuse interstellar bands in Upper Scorpius
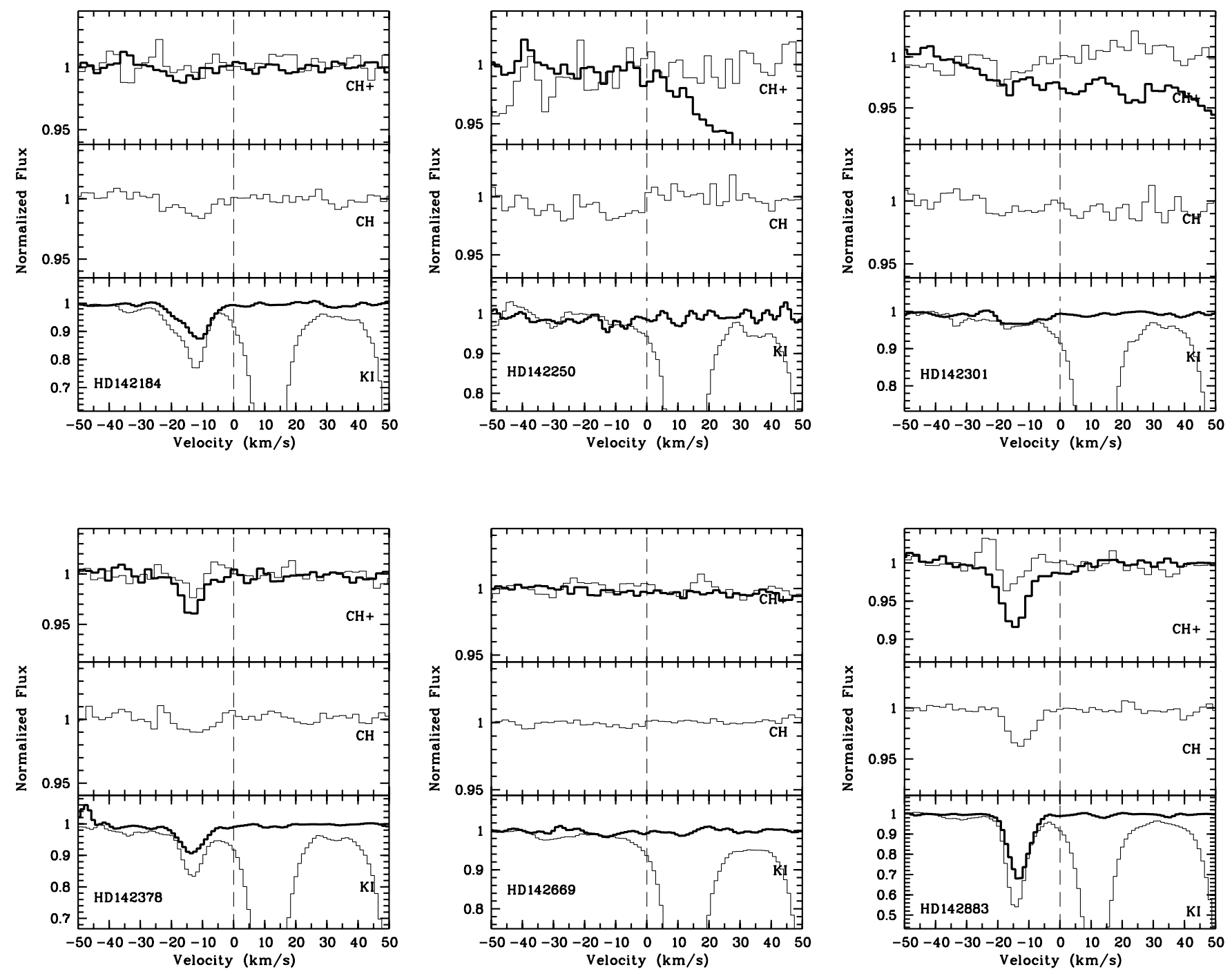

Fig. D.1. continued. 
A\&A 533, A129 (2011)
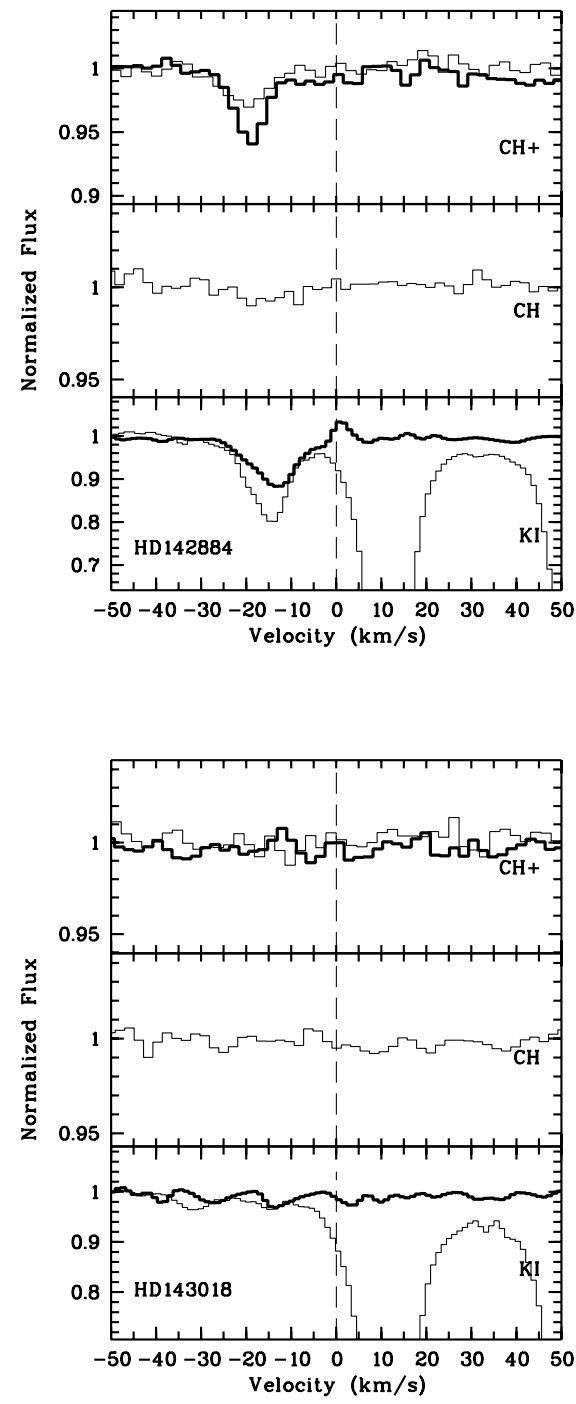

Fig. D.1. continued.
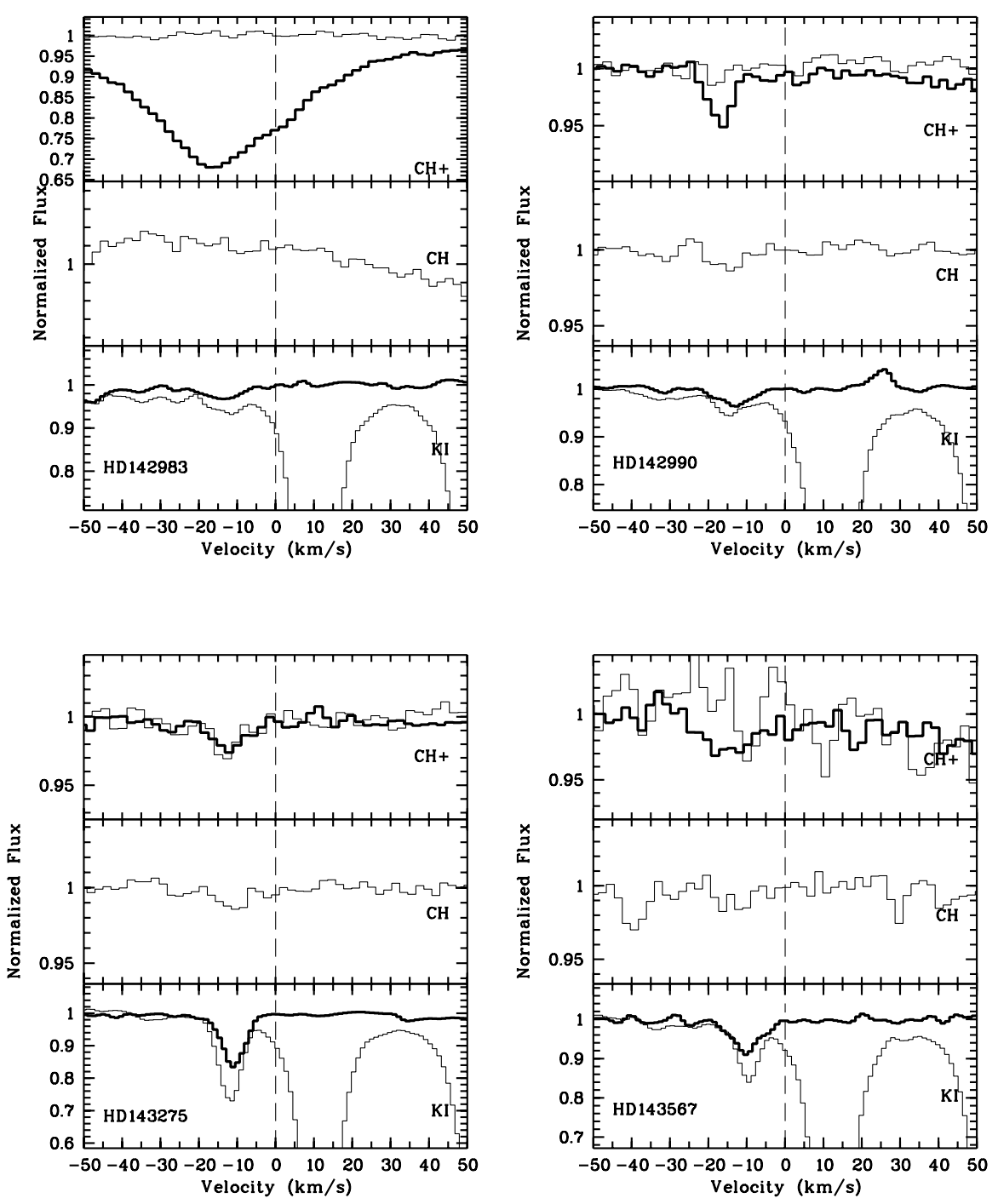
D. A. I. Vos et al.: Diffuse interstellar bands in Upper Scorpius
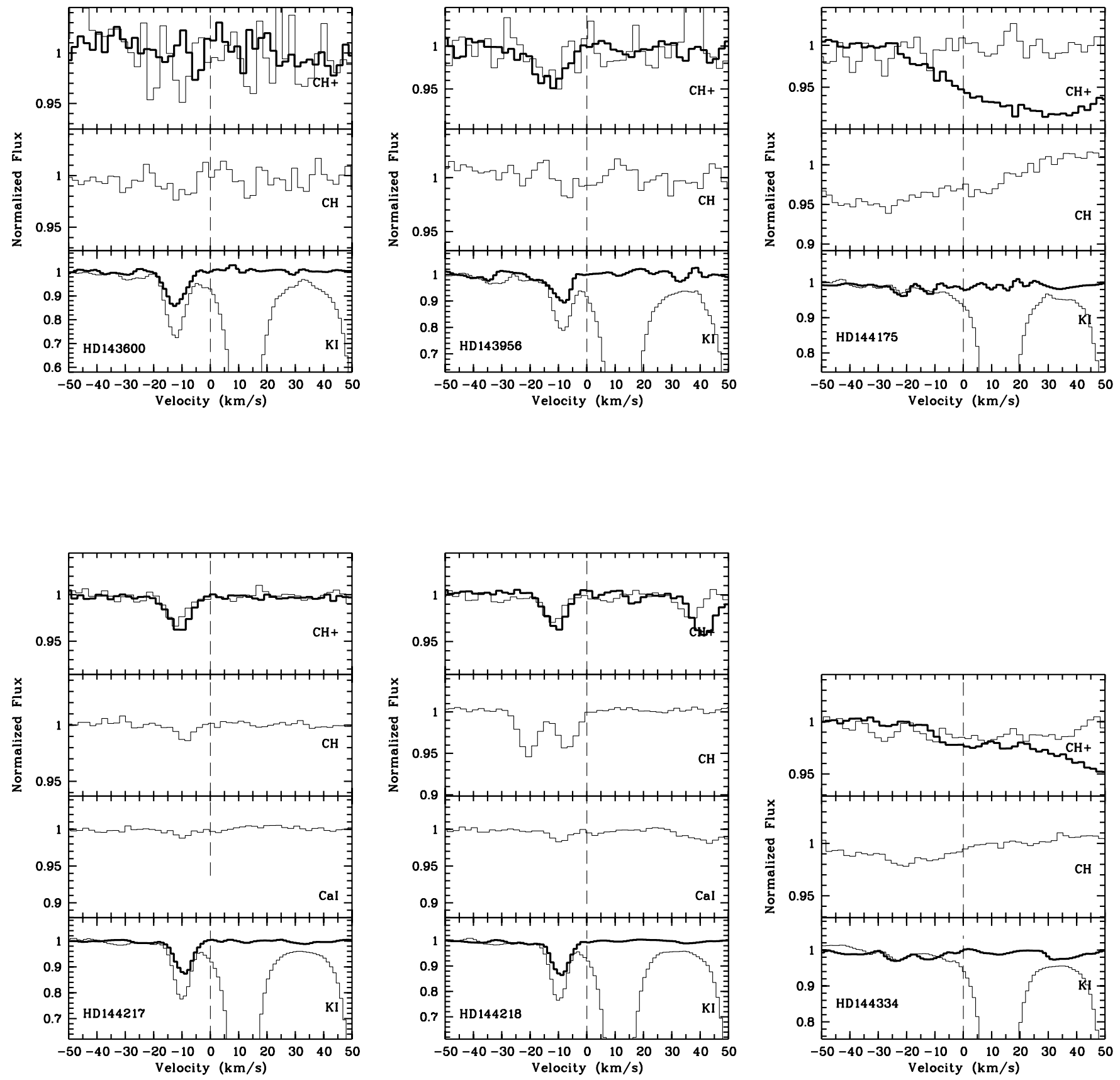

Fig. D.1. continued. 

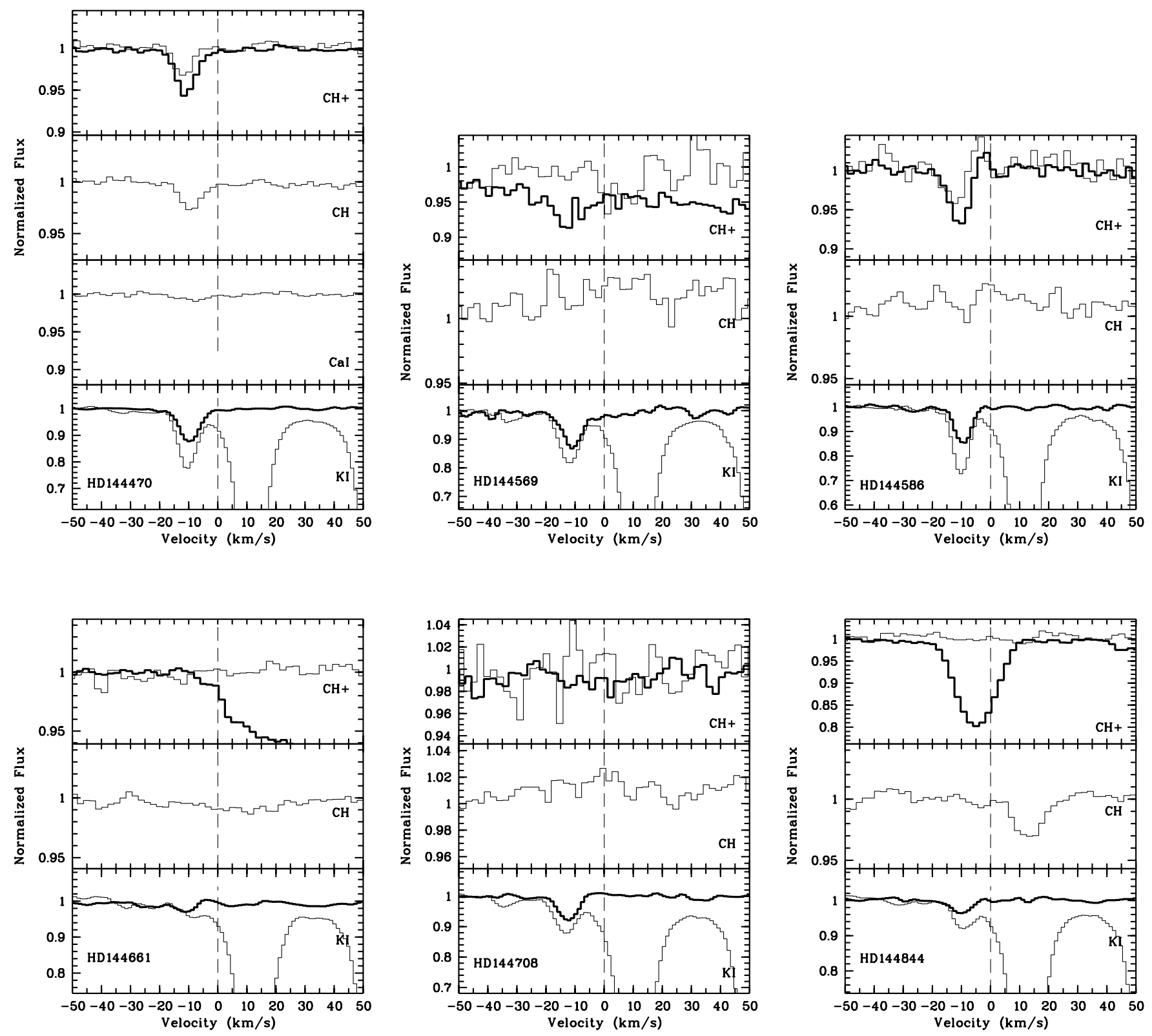

Fig. D.1. continued. 
D. A. I. Vos et al.: Diffuse interstellar bands in Upper Scorpius
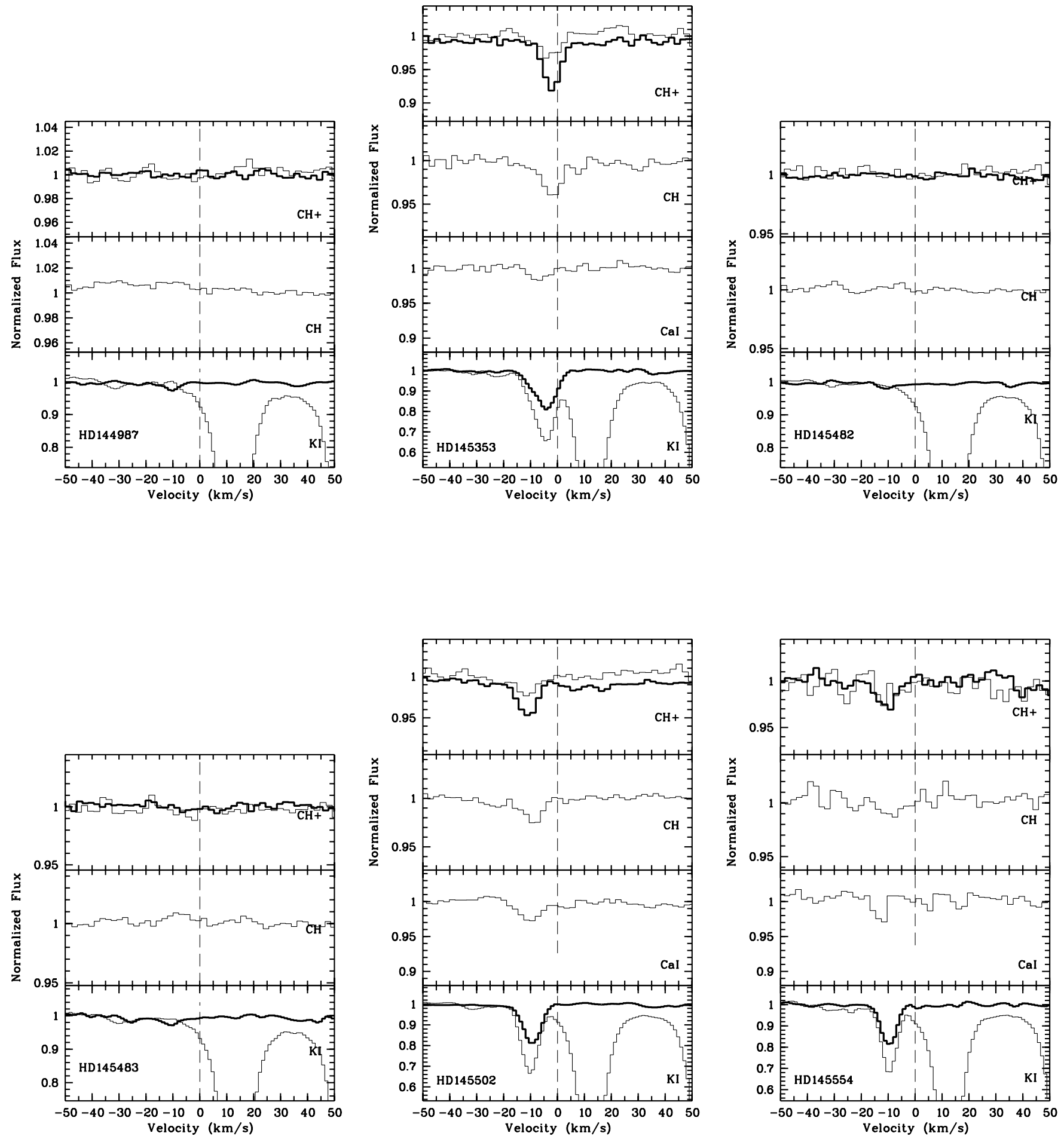

Fig. D.1. continued. 

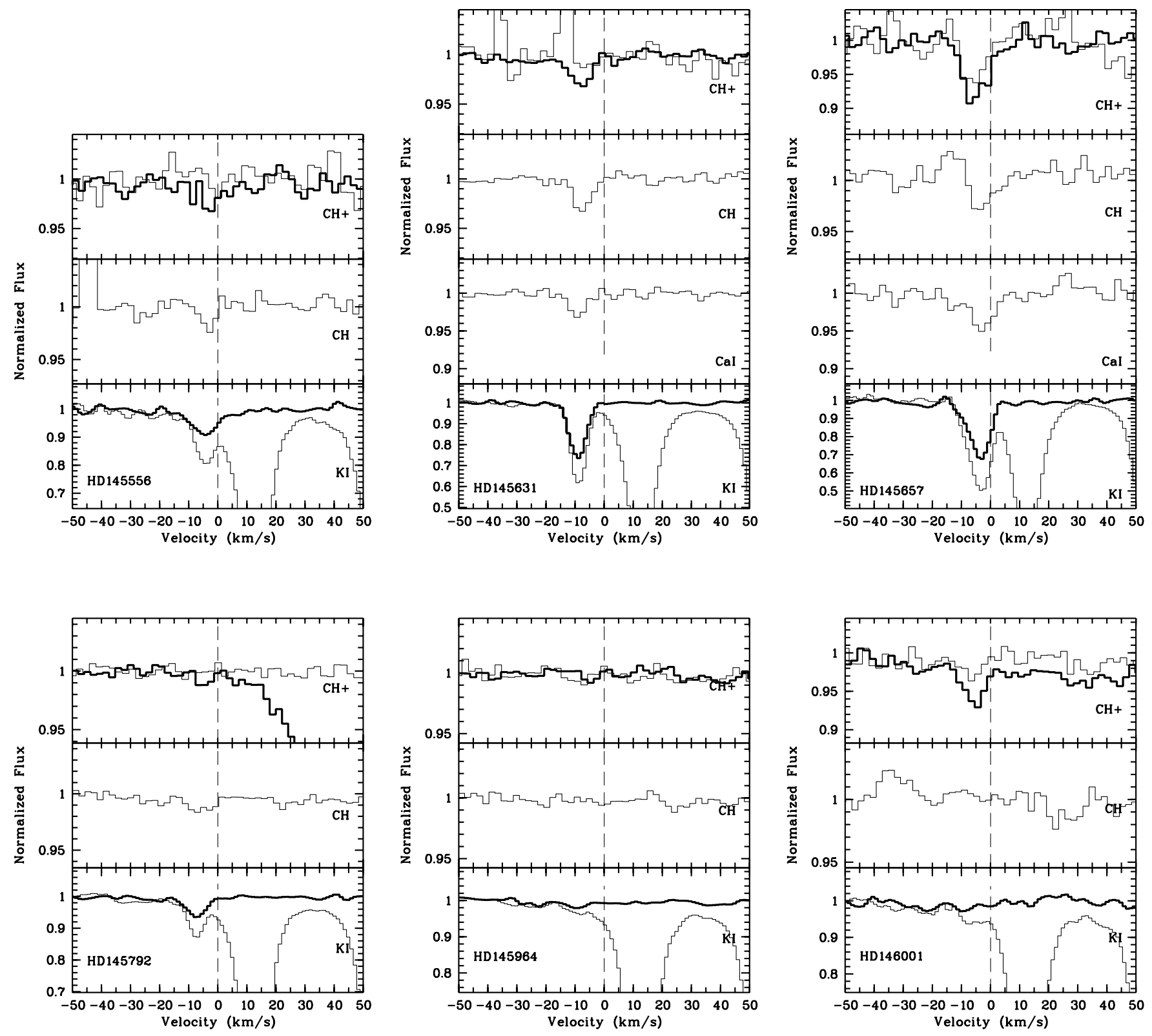

Fig. D.1. continued. 
D. A. I. Vos et al.: Diffuse interstellar bands in Upper Scorpius
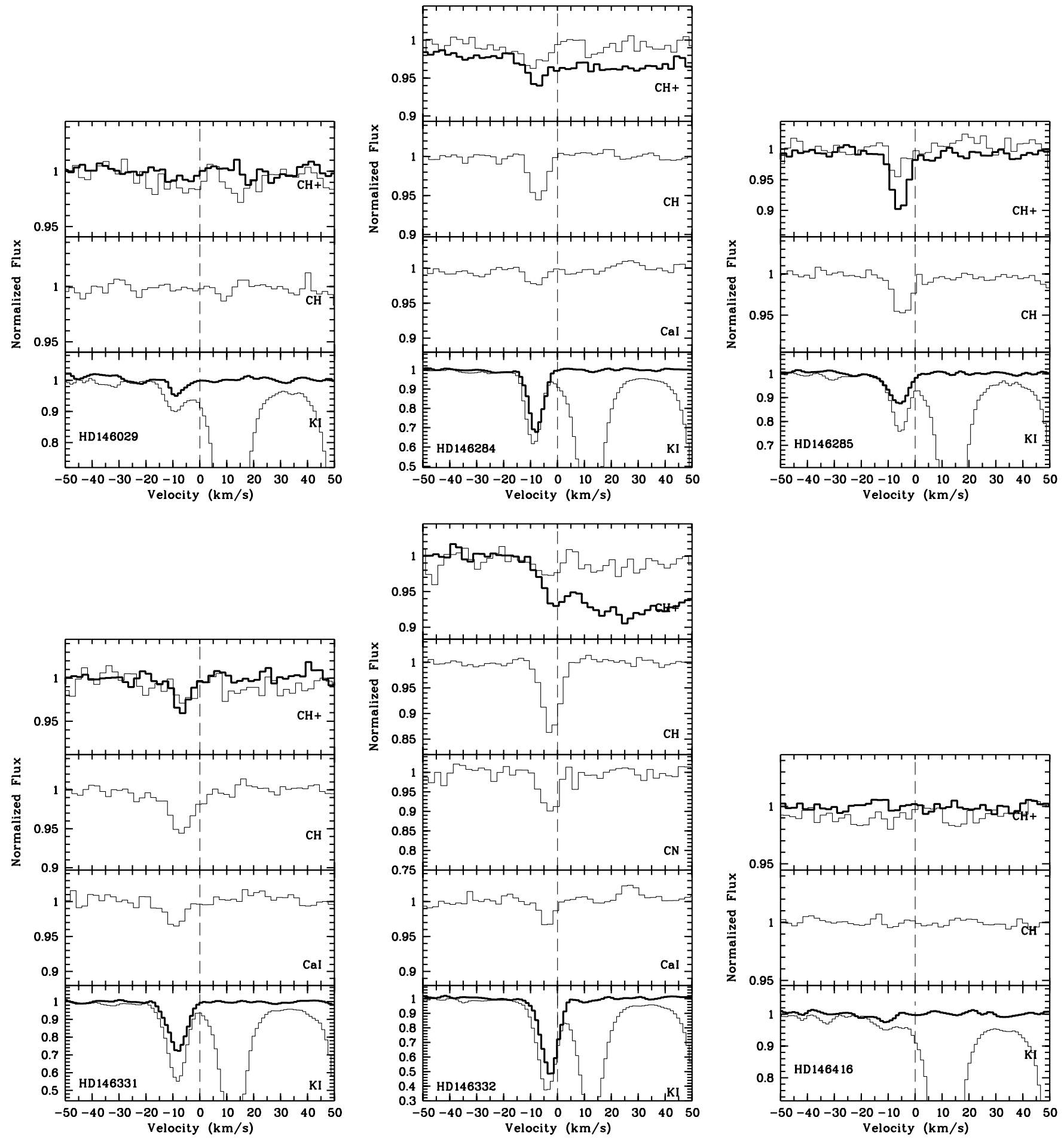

Fig. D.1. continued. 

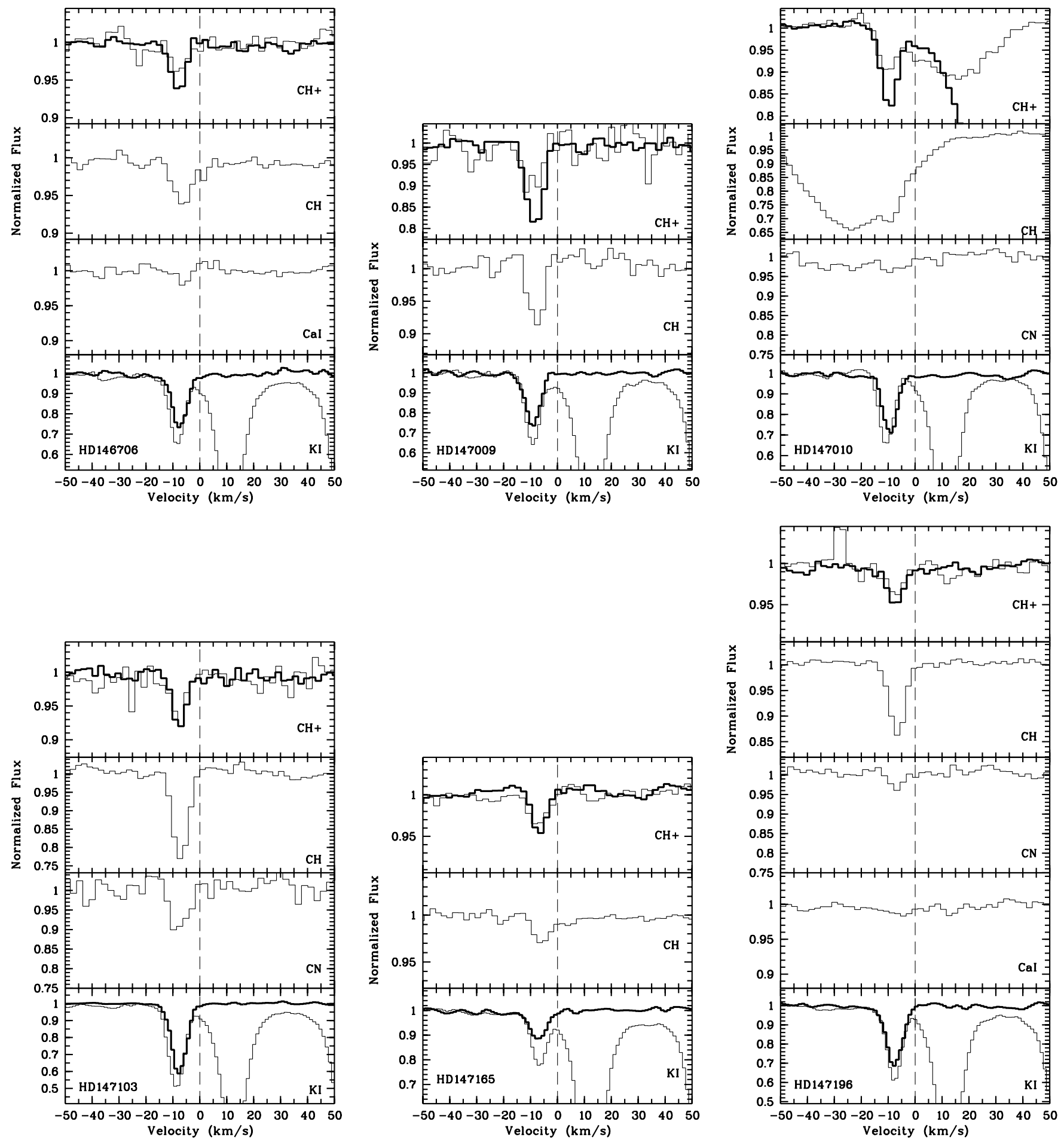

Fig. D.1. continued. 
D. A. I. Vos et al.: Diffuse interstellar bands in Upper Scorpius
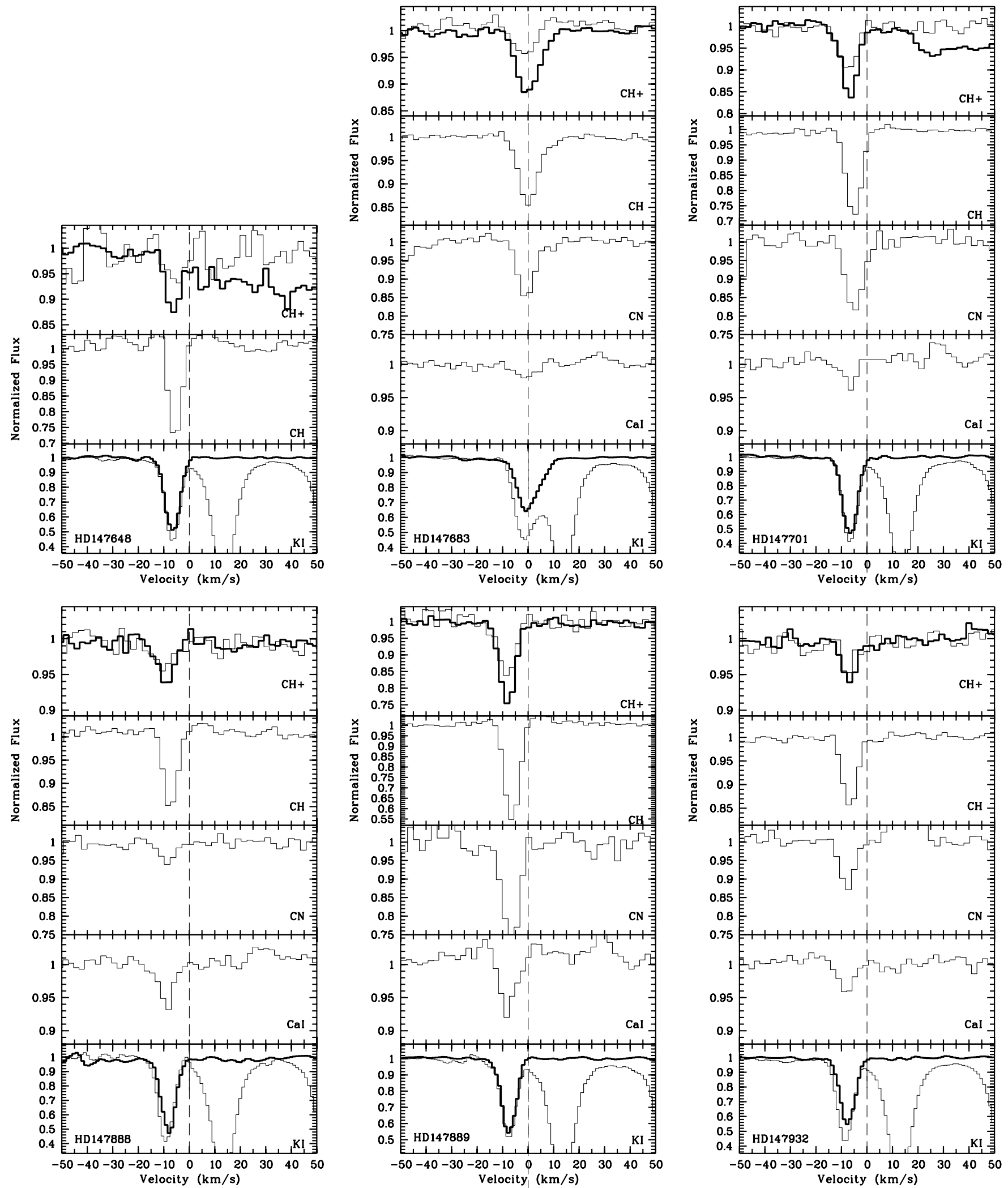

Fig. D.1. continued. 

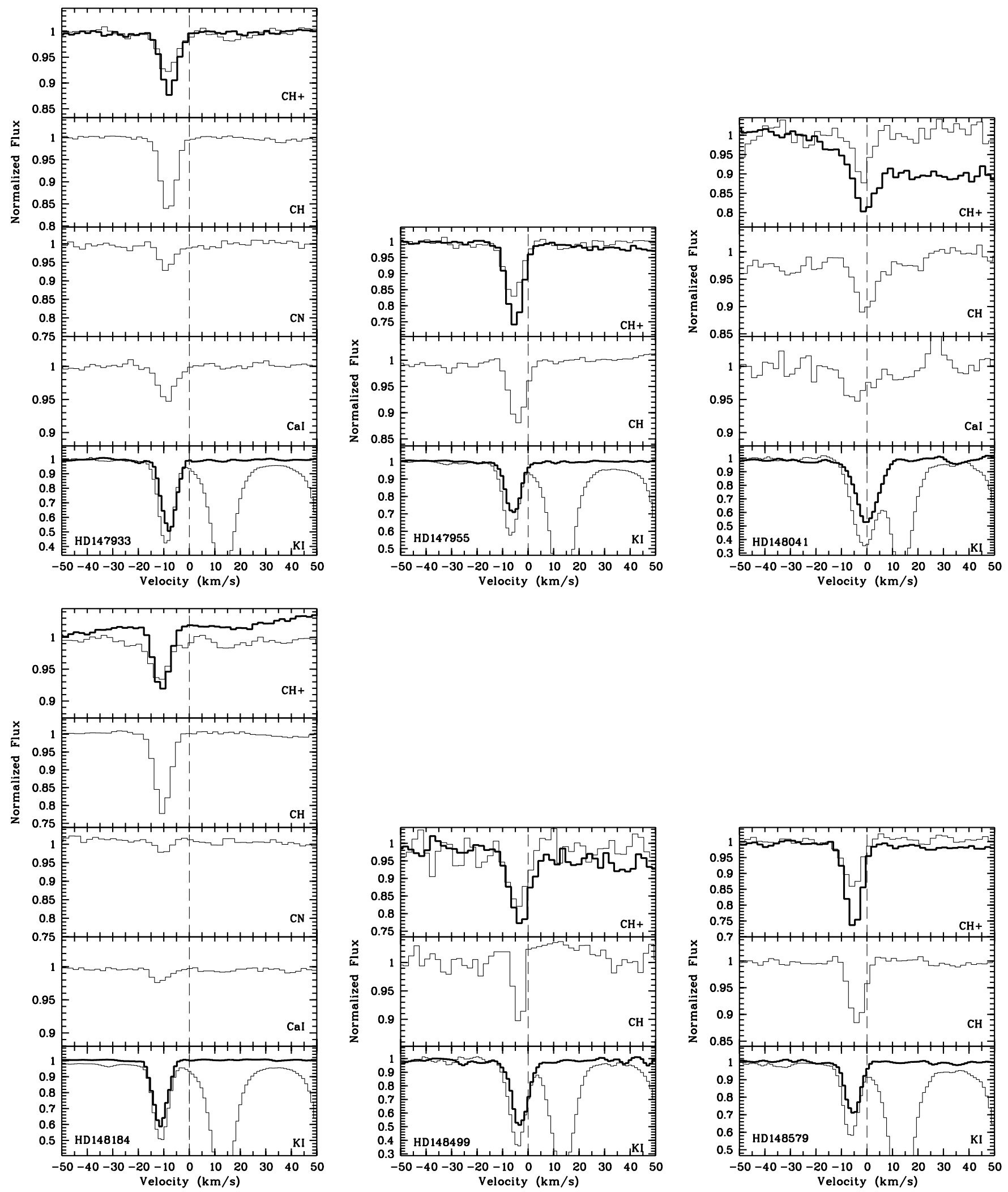

Fig. D.1. continued. 
D. A. I. Vos et al.: Diffuse interstellar bands in Upper Scorpius
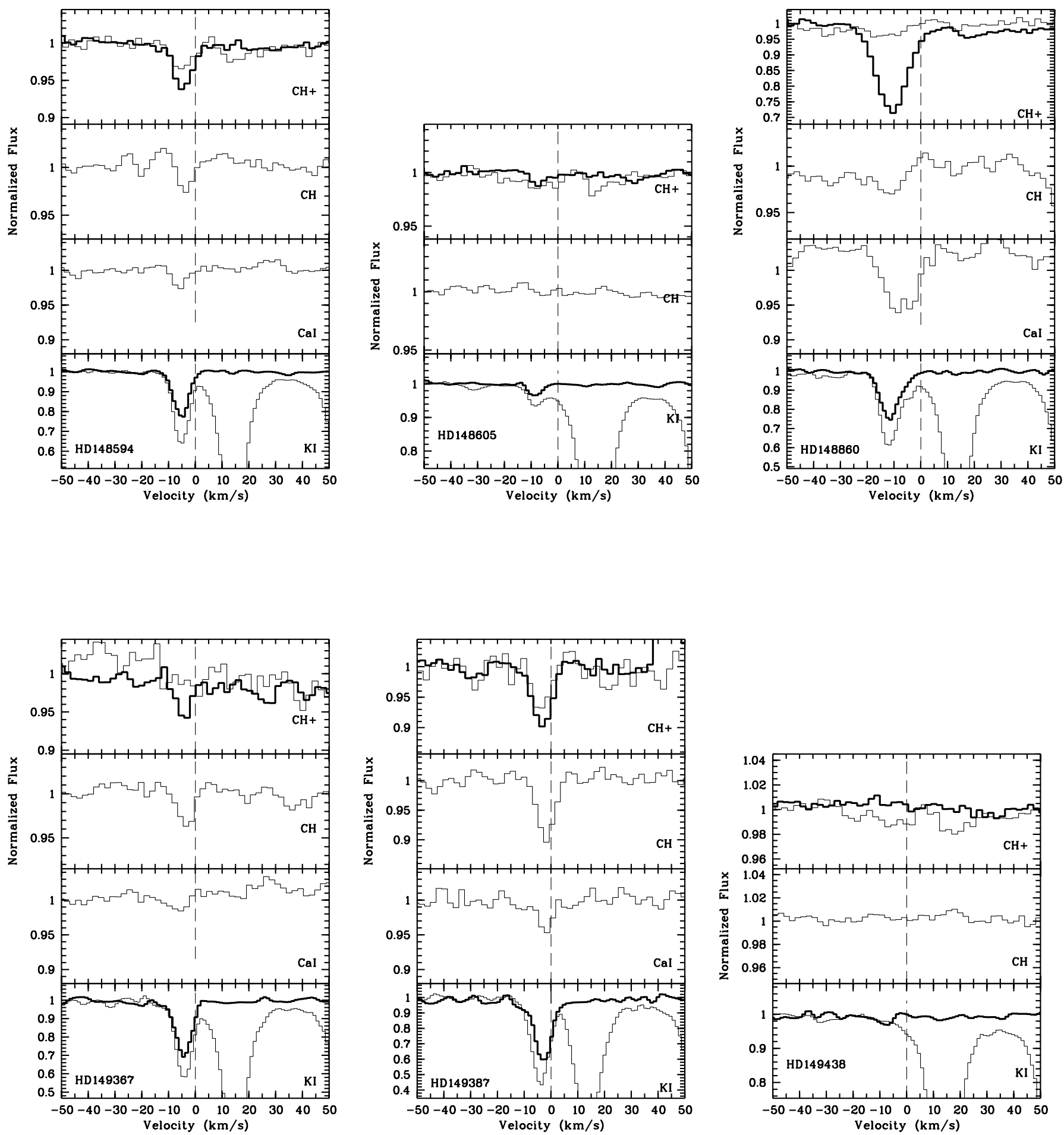

Fig. D.1. continued. 

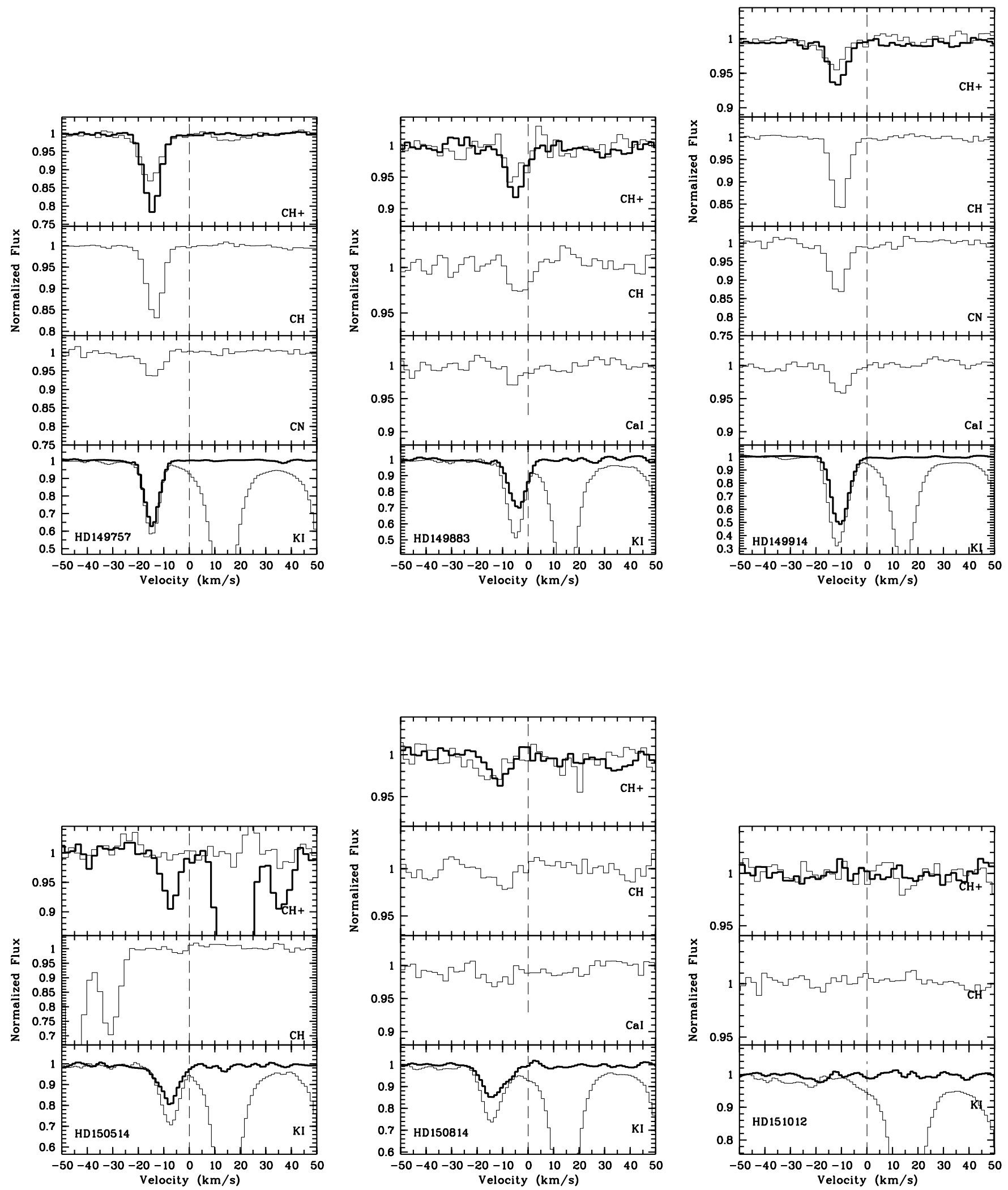

Fig. D.1. continued. 
D. A. I. Vos et al.: Diffuse interstellar bands in Upper Scorpius
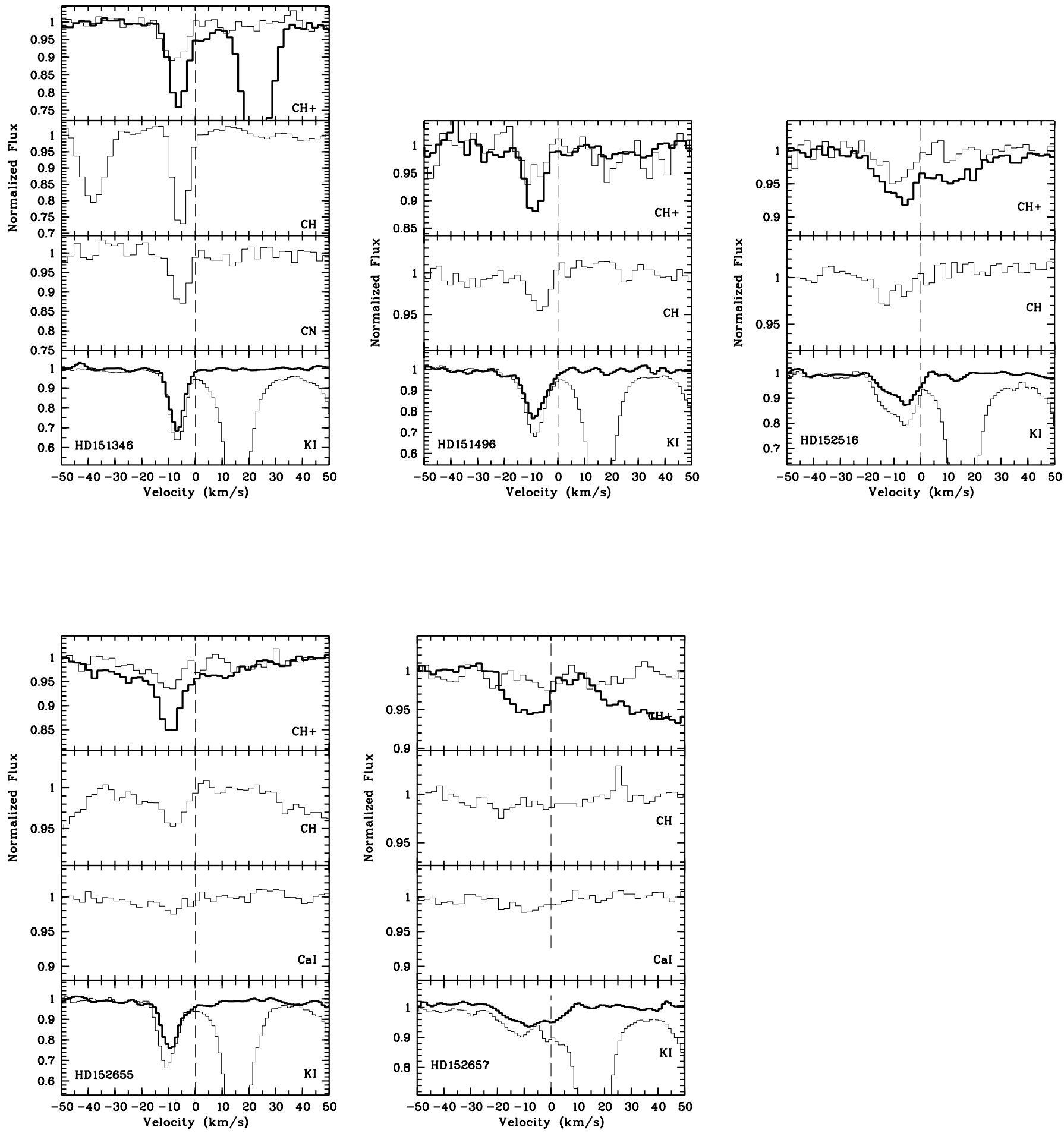

Fig. D.1. continued. 